\title{
WestVirginiaUniversity
}

THE RESEARCH REPOSITORY @ WVU

Graduate Theses, Dissertations, and Problem Reports

2000

\section{Studies of the respiratory chain of Methylococcus capsulatus (Bath)}

Timothy Brian Miley

West Virginia University

Follow this and additional works at: https://researchrepository.wvu.edu/etd

\section{Recommended Citation}

Miley, Timothy Brian, "Studies of the respiratory chain of Methylococcus capsulatus (Bath)" (2000).

Graduate Theses, Dissertations, and Problem Reports. 3193.

https://researchrepository.wvu.edu/etd/3193

This Dissertation is protected by copyright and/or related rights. It has been brought to you by the The Research Repository @ WVU with permission from the rights-holder(s). You are free to use this Dissertation in any way that is permitted by the copyright and related rights legislation that applies to your use. For other uses you must obtain permission from the rights-holder(s) directly, unless additional rights are indicated by a Creative Commons license in the record and/ or on the work itself. This Dissertation has been accepted for inclusion in WVU Graduate Theses, Dissertations, and Problem Reports collection by an authorized administrator of The Research Repository @ WVU.

For more information, please contact researchrepository@mail.wvu.edu. 


\title{
Studies of the Respiratory Chain of Methylococcus capsulatus (Bath)
}

\author{
Dissertation \\ Submitted to the School of Medicine \\ West Virginia University \\ In Partial Fulfillment of the Requirements for \\ The Degree of Doctor of Philosophy, Biochemistry \\ by \\ Timothy Brian Miley
}

\author{
Andrew Shiemke, Ph.D., Chair \\ Diana Beattie, Ph.D. \\ John Durham, Ph.D. \\ Mary Davis, Ph.D. \\ Vincent Castranova, Ph.D. \\ Department of Biochemistry \\ Morgantown, West Virginia
}

2000

Keywords: Methylococcus capsulatus, respiratory chain, methanotrophs 


\title{
ABSTRACT \\ Studies of the Respiratory Chain of Methylococcus capsulatus (Bath)
}

\begin{abstract}
Timothy Brian Miley
The methanotrophic bacterium, Methylococcus capsulatus, is capable of the conversion of methane to methanol at ambient temperature and pressure. In this manuscript, we show that quinols can provide reducing equivalents to the membranebound form of methane monooxygenase (pMMO), substituting for NADH in whole cells and membrane fractions. Additionally, quinols are shown to be effective reductants for the detergent-solubilized enzyme, whereas NADH is ineffective. The decyl-analog of plastoquinol and duroquinol provide the greatest MMO activity in whole cells and membrane fractions, as well as detergent-solubilized samples. Lauryl maltoside is by far the best detergent for solubilization of catalytically-active methane monooxygenase. This detergent was also utilized when reincorporating the endogenous quinone, 18'methylene $\mathrm{CoQ}_{8}$, during analysis of pMMO enzymatic activity supported by endogenous quinones. The endogenous quinone was capable of supporting activity of pMMO in detergent-solubilized fractions, when used in combination with NADH. However, the synthetically reduced form of the endogenous quinone is incapable of supporting activity in whole cells, membranes, or the solubilized fraction. Finally, oxygen uptake studies of whole cells, membranes, and solubilized membranes in addition to UV-visible spectroscopic studies reveal the existence of an alternative oxidase in vitro. The inhibitors octyl gallate and cyanide inhibit oxygen uptake in both membranes and whole cells.
\end{abstract}




\section{Table of Contents}

List of Abbreviations.......................................... vi

List of Illustrations/Figures.................................... viii

List of Tables..................................................

Chapter One: Introduction....................................... 1

Methylotrophs............................................2

Methanotrophs............................................ 2

The MMO System and Methylococcus capsulatus................5

Project Overview........................................ 11

References.......................................... 14

Chapter Two: Methods and Materials.............................23

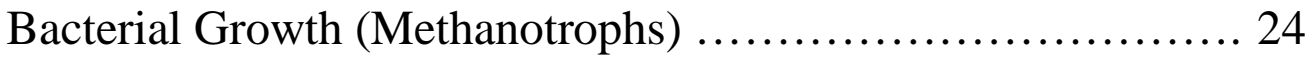

Cell Lysis and Membrane Isolation........................... 24

Enzymatic Activity Assays (pMMO Membrane Samples)...... 25

Detergent-solubilized pMMO Activity Assays ................ 26

Preparation of Quinols.................................... 26

Octanol-water Partition Coefficients of Exogenous Quinols.... 27

pMMO Detergent Solubilization .......................... 27

Acetylene Labeling of pMMO........................... 28

Gel Electrophoresis.................................... 28 
Oxygen Uptake Studies

Isolation, Purification and Characterization of Endogenous

Quinones............................................. 30

Spectroscopic Kinetic Studies........................... 31

References........................................... 32

Chapter Three: Detergent Solubilization of Membrane-bound Methane

Monoxygenase Requires Plastoquinol Analogs as Electron

Donors...................................................... 33

Abstract................................................... 34

Introduction............................................ 35

Results................................................. 38

Discussion............................................ 47

Acknowledgement........................................ 51

References.............................................. 52

Chapter Four: Isolation, Purification, and Characterization of

Endogenous Quinones and Their Use in Support of pMMO

Activity in vitro....................................... 56

Introduction.......................................... 57

Results............................................. 59

Discussion............................................. 75 
Acknowledgement......................................... 79

References................................................. 80

Chapter Five: Oxygen Uptake Studies and Spectroscopic Kinetics.... 83

Introduction........................................... 84

Results................................................. 86

Discussion................................................ 98

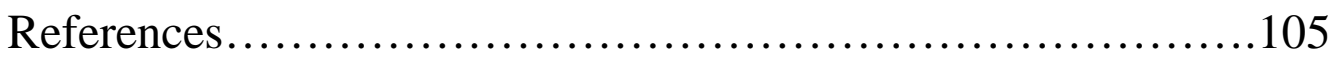

Chapter Six: Conclusions......................................... 109

References.......................................... 115

Future Work................................................. 117 


\section{LIST OF ABBREVIATIONS}

AMO: Ammonia monooxygenase

BCA: Bicinchoninic acid

$\mathrm{CN}$ : cyanide

cDNA: Complementary DNA

DCE: Dichloroethylene

DMSO: Dimethyl sulfoxide

Dnase I: Deoxyribonuclease I

EDTA: Ethylenediamine tetraacetic acid

EPR: Electron paramagnetic resonance

FAD: Flavin adenine dinucleotide

kDa: kilodalton

MMO: Methane monooxygenase

MMOB: Regulatory component of sMMO

MMOH: Hydroxylase component of sMMO

MMOR: Reductase component of sMMO

NAD+: Oxidized form of NADH

NADH: Nicotinamide adenine dinucleotide (reduced form)

NDH-2: Type 2 NADH-quinone oxidoreductase

NMS: Nitrate mineral salts medium

OG: Octyl gallate

PIPES: 1,4-piperizine diethane sulfonic acid

pMMO: Membrane-bound form of MMO 
$\mathrm{Q}_{0}$ : Ubiquinone 0 (no isoprene tail)

$\mathrm{Q}_{1}$ : Ubiquinone 1 (one isoprene in tail)

$\mathrm{Q}_{2}$ : Ubiquinone 2 (two isoprenes in tail)

Q6: Ubiquinone 6 (six isoprenes in tail)

Q7: Ubiquinone 7 (seven isoprenes in tail)

Q8: Ubiquinone 8 (eight isoprenes in tail)

$\mathrm{Q}_{9}$ : Ubiquinone 9 (nine isoprenes in tail)

$\mathrm{Q}_{10}:$ Ubiquinone 10 (ten isoprenes in tail)

SCFH: Standard cubic feet per hour

SDS-PAGE: Sodium dodecyl sulfate-polyacrylamide gel electrophoresis

SHAM: Salicylhydroxamic acid

sMMO: Soluble form of MMO

TCE: Trichloroethylene

TMPD: Tetramethyl phenylenediamine

TRIS: Tris[hydroxymethyl]-aminomethane

VC: vinyl chloride 


\section{LIST OF ILLUSTRATIONS/FIGURES}

\section{Chapter One}

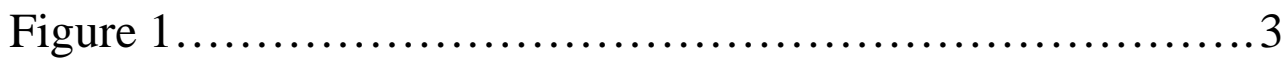

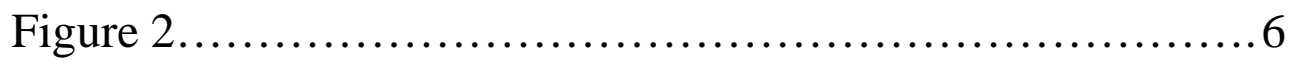

Chapter Three

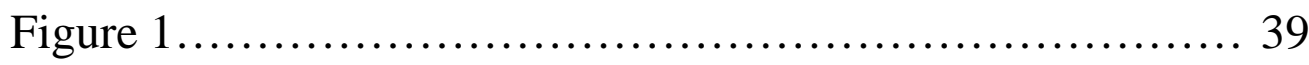

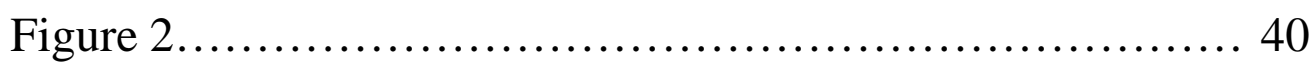

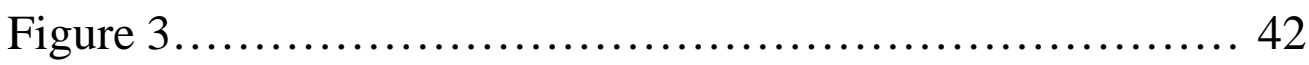

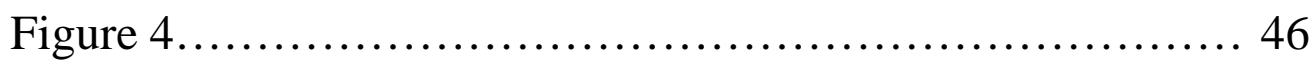

Chapter Four

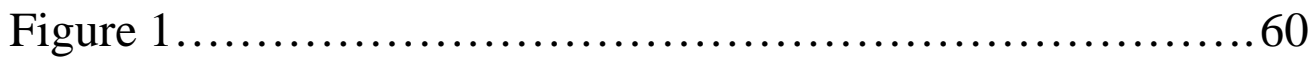

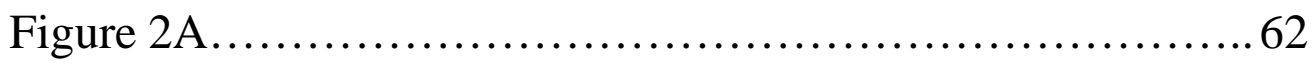

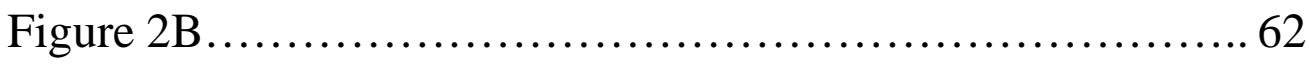

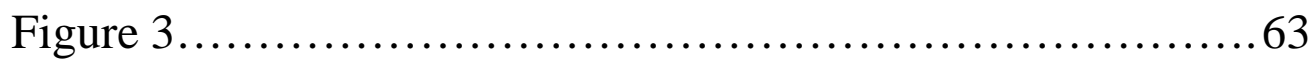

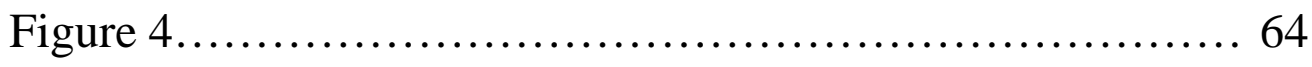

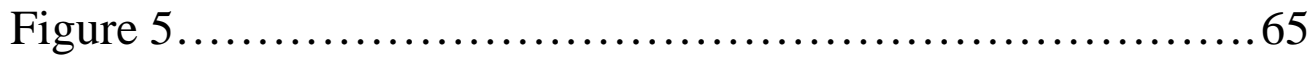

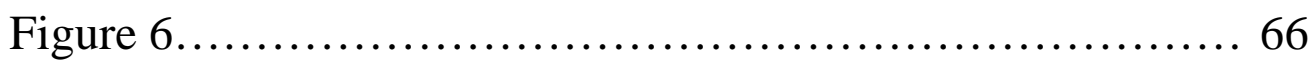

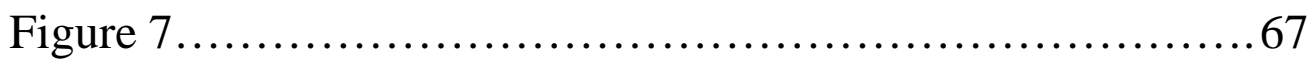

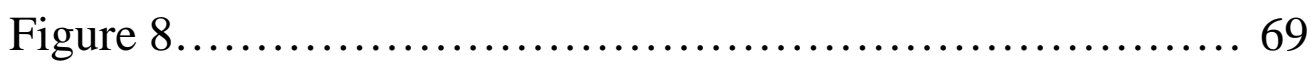

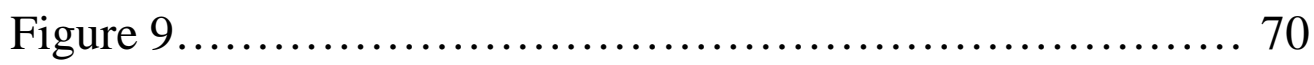




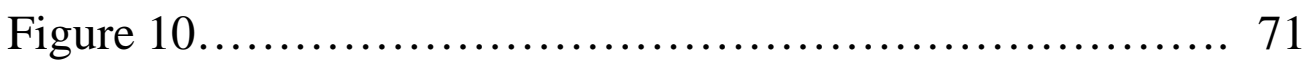

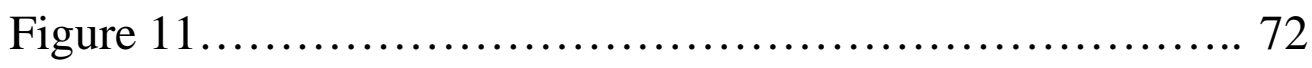

\section{Chapter Five}

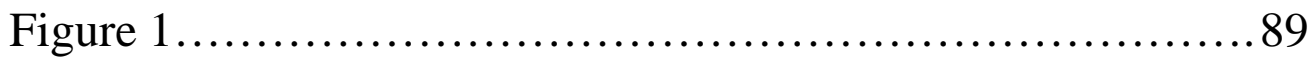

Figure 2A.................................................... 91

Figure 2B................................................... 91

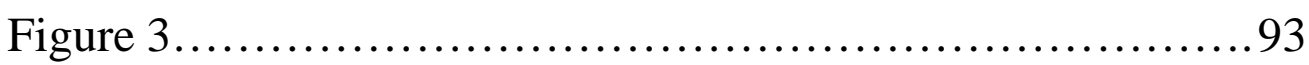

Figure 4A.................................................. 94

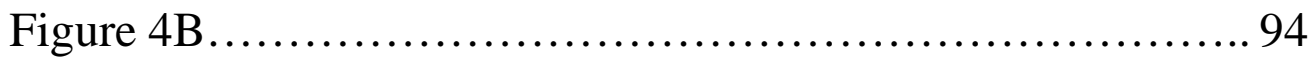

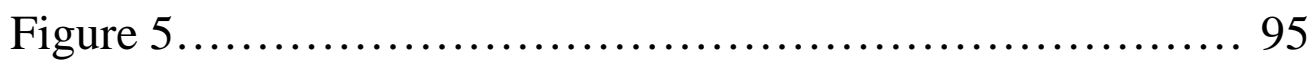

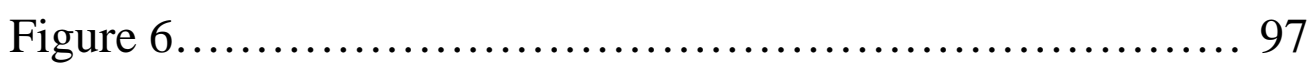

\section{Chapter Six}

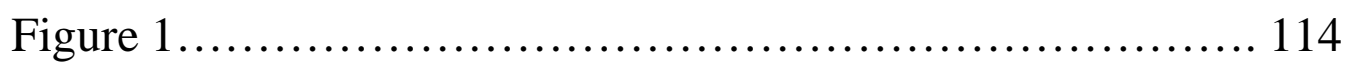




\section{LIST OF TABLES}

Chapter Three

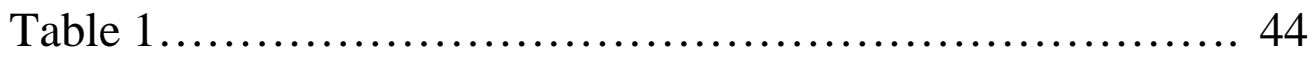

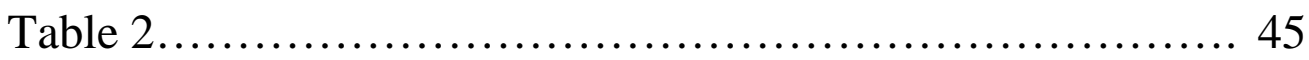

Chapter Four

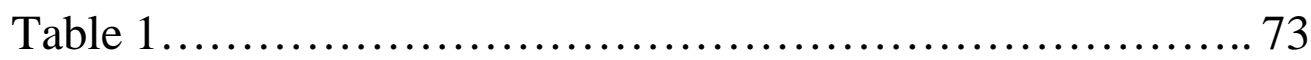

Chapter Five

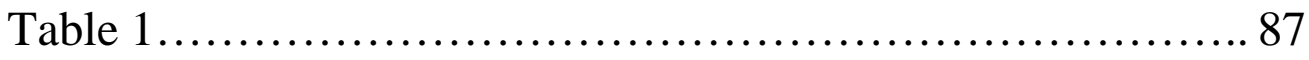

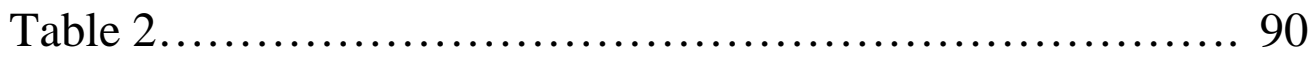




\title{
CHAPTER ONE
}

\author{
Introduction
}




\section{Methylotrophs}

Methylotrophs are a diverse group of eubacteria that utilize reduced carbon substrates possessing no carbon-carbon bonds as their sole source of carbon and energy (5). More specifically, this includes bacteria which can utilize methane, methanol, methylated amines, or methylated sulfur compounds as their substrates for growth. Most methylotrophs studied are gram-negative and obligately methylotrophic, but gram positive species have been described. Also, a variety of facultative organisms are known which are capable of growing on either one-carbon compounds or multi-carbon compounds. Many methylotrophs are aerobic while a few are anaerobic (32). Furthermore, methylotrophs are widespread throughout nature, existing at various interfaces where both oxygen (aerobic) and carbon sources are both plentiful (27) such as soils, groundwater, oceans and freshwater sediments (62).

\section{Methanotrophs}

Methanotrophs are a diverse group of organisms comprising several genera and perhaps more than one family (37). These organisms use methane as their sole source of both carbon and energy $(6,37)$. Methanotrophs reside in the family Methylococcaceae and include gram-negative rods, vibrio, and cocci (26). They are strictly aerobic, are catalase and cytochrome $c$ oxidase positive, and possess cytochromes $c, a$ and $o$ or $b(26)$. They are quite widespread in nature, existing in soil, fresh water or marine environments and have been identified in symbiotic relationships with marine bivalves (2). All strains also possess a complex arrangement of intracytoplasmic membranes (57) when grown on methane (37) (Figure 1). Members of this group also have the ability to form exospores, cysts, or lipid cysts during resting stage $(27,37)$. Additionally, methanotrophic bacteria 


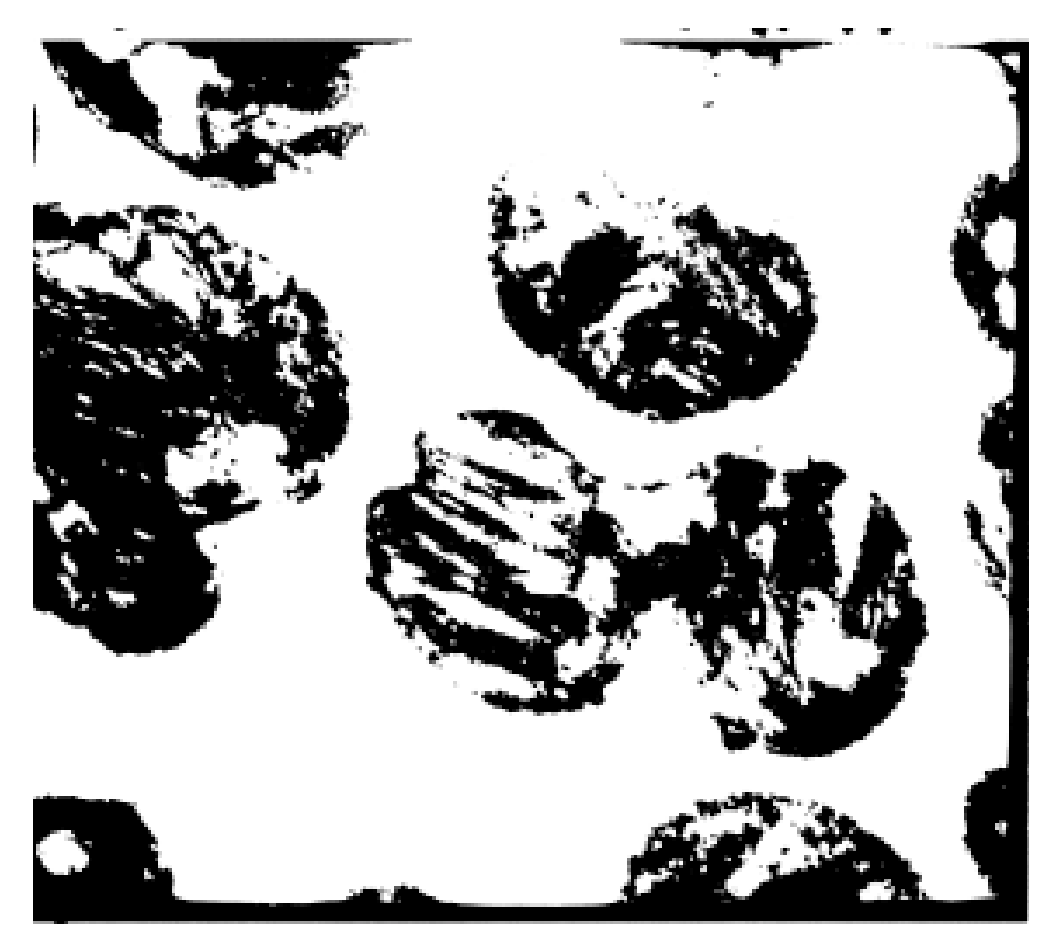

Figure 1

Electron micrograph of Methylococcus capsulatus magnified 50,000X detailing the intracytoplasmic membranes indicative of pMMO expression. 
are a vital part of the global carbon cycle, representing the primary means for the production of carbon dioxide from methane, an end product of anaerobic fermentation (26). In some aquatic environments they are found at certain times of the year within a very restricted range of depths, where methane discharged from anaerobic degradation and dissolved oxygen are at levels optimal for their growth.

Methane-utilizing bacteria have been split into two broad groups designated Type I and Type II, on the basis of their carbon assimilation pathways, and on the arrangement of their intracytoplasmic membranes $(6,57)$. The carbon assimilation pathways consist of the ribulose monophosphate (RuMP) pathway and the serine pathway. Type I methanotrophs are characterized by the former pathway, while Type II methanotrophs utilize the latter $(6,15)$. The organism under study in this laboratory for the past nine years, Methylococcus capsulatus (Bath), is capable of nitrogen fixation but prefers nitrates as its source of nitrogen (37). M. capsulatus is unique in that it contains both a RuMP pathway as well as a serine pathway, and it is also capable of autotrophic carbon dioxide fixation via the Calvin-Benson cycle $(27,63)$. Due to these complexities, one of the major groups was further subdivided to accommodate this unique prokaryote and designated Type X. It is one of only a handful of methanotrophs isolated and described in the literature before 1970. Methylococcus capsulatus will be the focus of the research described here. 


\section{The MMO System and Methylococcus capsulatus}

The bioenergetics and metabolism of methanotrophs hinge on the methane monooxygenase (MMO) system. It is now known that two forms of this enzyme exist. There is a particulate (membrane-bound) form, or pMMO, as well as a soluble (sMMO) form. The two forms are not expressed at the same time during growth. Consequently, the environmental growth conditions dictate which form is expressed at any given time $(23,45,57)$. The soluble form of the enzyme is expressed at high cell density and relatively low copper concentrations, and further stimulated by iron (22). Additionally, the gene cluster for sMMO from $M$. capsulatus has been shown to be transcriptionally repressed in vitro by the presence of copper ions (39). Conversely, methanotrophic bacteria grown to normal cell densities $(2-5 \mathrm{~g} / \mathrm{L})$ express only pMMO, and this is further enhanced by the presence of copper $(24,57$ ) (Figure 2). It appears that only three methanotroph strains can express the soluble form. Therefore, the particulate form is believed to be the most prevalent in nature. Even though the two forms of the enzyme catalyze the same reaction, they are functionally different. The soluble MMO can oxidize cyclic and aromatic compounds, whereas pMMO can oxidize alkanes and alkenes only up to four carbons in length and will not oxidize cyclic or aromatic compounds (15).

The soluble form of the enzyme has been purified from Type II methanotrophs as well as $M$. capsulatus (22). The sMMO from $M$. capsulatus (Bath) is the most thoroughly studied and best characterized at this point. It consists of three separate components known as $\mathrm{A}, \mathrm{B}$, and $\mathrm{C}$. Component $\mathrm{A}$ is a hydroxylase $(\mathrm{MMOH})$ and consists of three subunits with molecular weights of $17 \mathrm{kD}, 42 \mathrm{kD}$, and $54 \mathrm{kD}$. The arrangement of these subunits is of an $\alpha_{2} \beta_{2} \gamma_{2}$ stoichiometry. Crystallographic 


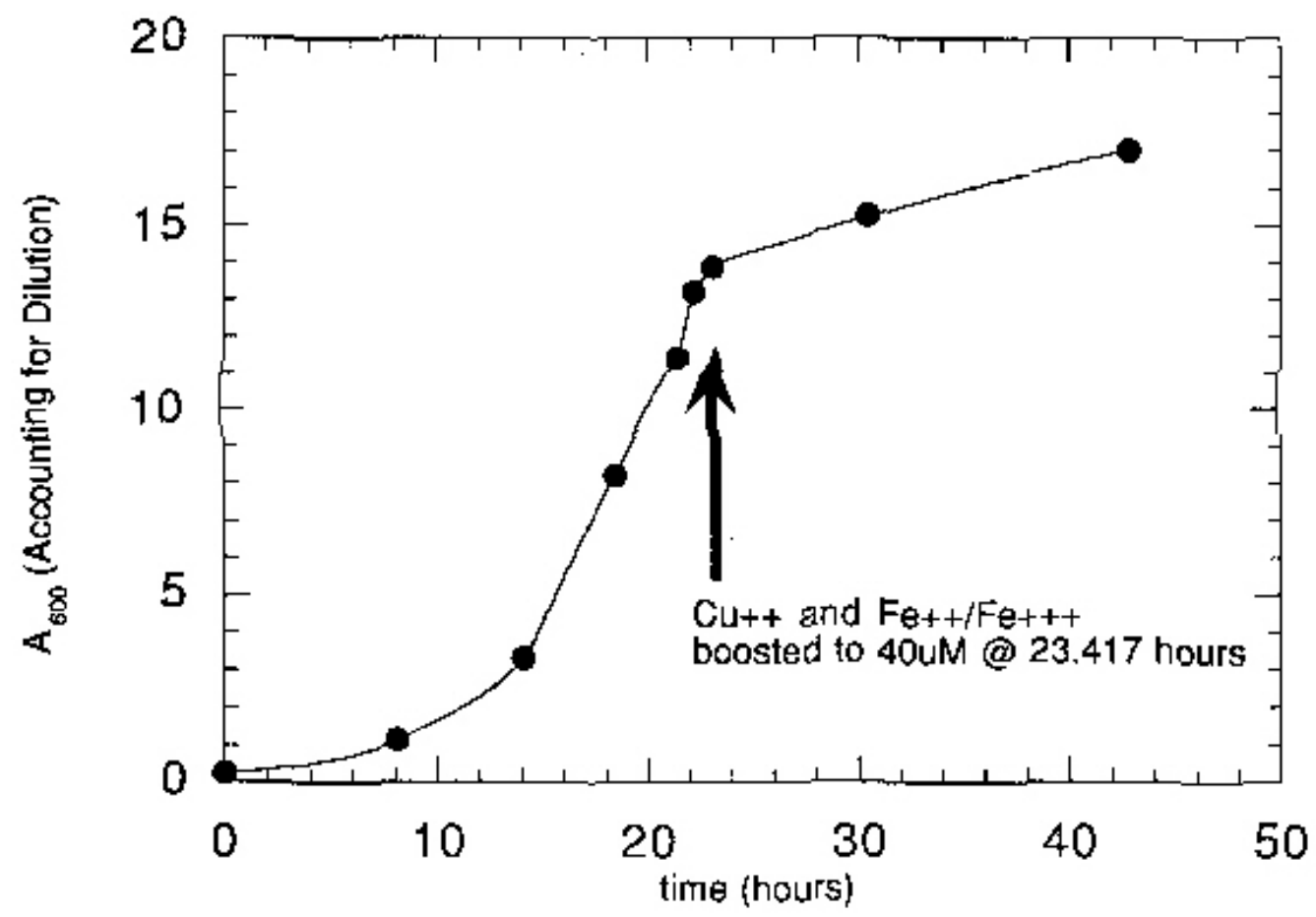

Figure 2

Typical growth curve for a culture of Methylococcus capsulatus following transfer to the fermentation vessel. Copper and iron salts were added at the time point indicated. This particular batch was the initial batch of three grown succesively from one inoculation. Typical growth rates for batches inoculated with small amount of initial batch are approximately two-fold faster. 
characterization of the enzyme demonstrated the presence of a $\mu$-oxo bridged iron cluster in the active site of the hydroxylase, with binuclear iron active sites found in each $\alpha$ subunit (22).

Both forms of MMO are irreversibly inhibited by acetylene $(9,45)$ and both are also inhibited by the oxygen analogs azide and cyanide (9). The membrane-bound form is also inhibited by metal chelators such as EDTA and dithiocarbamates whereas sMMO shows no inhibitory effects due to the presence of these compounds $(6,9)$. This suggests that the putative metal center(s) of pMMO are not tightly bound.

Transient kinetics have been utilized to detect several reactive oxygen intermediates during oxygen activation by sMMO (30). The fully reduced diferrous state of the enzyme exhibits an EPR signal of $\mathrm{g}=16$ (22). This signal disappears upon addition of oxygen with a rate constant of $22 \mathrm{~s}^{-1}$. The decay results in the formation of an EPR silent compound Q, which absorbs in the UV-Vis range at 330 and $430 \mathrm{~nm}$. Results from both EXAFS and Mossbauer spectroscopy have suggested that the Q intermediate is an $\mathrm{Fe}^{\mathrm{IV}} \mathrm{O}_{2}$ diamond core structure (52)

Component $\mathrm{B}(\mathrm{MMOB})$ is a relatively small protein apparently absent of cofactors. Recently published investigations have suggested that MMOB plays a regulatory role in the sMMO mechanism. When MMOB is present, $\mathrm{v}_{0}$ (methane oxidation) is increased up to 150 -fold (23). Published reports utilizing the MMO from Methylosinus trichosporium OB3b have shown that MMOB causes a $-132 \mathrm{mV}$ shift in the midpoint potential of MMOH as measured by both EPR and Mossbauer spectroscopy. This seems to suggest that the B subunit binds approximately 10,000-fold more strongly to the diferric state of $\mathrm{MMOH}$ than to the diferrous state (42). Recent EPR evidence 
shows that this change in midpoint potential is related to a change in the conformation of the monooxygenase diiron cluster (17). The conformational change is related to a concomitant protonation of a bridging $\mu$-hydroxyl ligand. This results in weakening of the bond and release of a bridging water ligand from the diferrous $\mathrm{MMOH}$, thus allowing oxygen binding and activation.

Component $\mathrm{C}$ of sMMO (MMOR) contains Fe-S clusters as well as a flavin. It mediates electron flow from the reductant (intracellular NADH) to $\mathrm{MMOH}(9)$.

The gene for the hydroxylase component of sMMO has been cloned from $M$. capsulatus (Bath) $(54,55)$. Additionally, hybridization experiments have been performed with cDNA libraries from various methanotrophs. Positive hybridizations have been observed with only M. capsulatus and two strains of Methylosinus (56). This provides evidence that the sMMO and pMMO are products of separate genes, and that pMMO is the more prevalent of the two.

The entire gene for the sMMO complex has been sequenced and all of the genes for sMMO have been located in one cluster (operon) in both Methylosinus trichosporium OB3b (12) and $M$. capsulatus $(54,55)$. The order of the cluster is mmoX, mmoY, mmoB, mmoZ, orfY and mmoC. The mmoX, mmoY, and mmoZ genes encode the $\alpha, \beta$, and $\gamma$ subunits of $\mathrm{MMOH}$, respectively. Conversely, $\mathrm{mmoB}$ and mmoC encode the MMOB and MMOC peptides. The open reading frame orf Y serves no known function.

In contrast to the soluble form of methane monooxygenase, the particulate form is less well-characterized. It has yet to be reproducibly purified in an active form; this is primarily due to the lability of the enzyme. Many laboratories report enzyme inactivation upon cell lysis (H. Dalton, personal communication; J. Lipscomb, personal 
communication; M. Donelly, personal communication). Additionally, when pMMO activity is stabilized in vitro, solubilization by most detergents results in loss of activity.

There have been four reports, thus far, in the literature claiming purification of pMMO and they differ, somewhat in the methods utilized $(1,58,66)$. However, we and others have been unable to reproduce the results of the earlier two, while the latter two result in purification of a largely inactive protein. Overall, the purification schemes for pMMO are, at best, doubtful.

The most reliable information on pMMO has been gained via membrane suspensions, cell extracts, or whole cells. It has been shown that copper stabilizes and increases the activity of pMMO in membrane fractions $(38,46,57)$. Without exogenous copper, the stability of pMMO activity decreases markedly following cell lysis. However, if the lysis buffer is supplemented with copper, pMMO can retain its activity for several weeks (38).

There have also been previous reports of the appearance of three novel polypeptides on SDS-PAGE following the expression of pMMO. These polypeptides are not present when the soluble form of methane monooxygenase is expressed. These three polypeptides have apparent molecular weights of $46 \mathrm{kD}, 35 \mathrm{kD}$, and $26 \mathrm{kD}(46,57)$. The $26 \mathrm{kD}$ protein is specifically labeled upon exposure to ${ }^{14}[\mathrm{C}]$-acetylene due to the fact that acetylene is a suicide substrate for MMO. Therefore, the $26 \mathrm{kD}$ polypeptide is presumed to contain the substrate-oxidation site of pMMO. Acetylene achieves similar inhibition with cytochrome $\mathrm{P} 450$ by binding to the heme at its substrate-oxidation site (41).

A cDNA probe has been made to the $45 \mathrm{kD}$ peptide and has been used to screen for pMMO genes in several strains (50). From these studies, it has been discovered that 
the $26 \mathrm{kD}$ polypeptide gene lies upstream of the gene for the $45 \mathrm{kD}$ polypeptide. Also, these genes have been shown to be similar to the amoB and amoA genes of ammonia monoxygenase. These genes in AMO express similar molecular weight polypeptides. Finally, AMO is also stabilized by copper addition, inhibited by acetylene and contains a subunit irreversibly labeled by this acetylene (the molecular weight of this subunit is $27 \mathrm{kD})(21)$.

This laboratory, as well as others, has reported that the enzymatic activity of pMMO is stimulated by the addition of exogenous copper. At present, the two most likely explanations concerning this effect are: (1) copper causes more pMMO to be produced at a transcriptional level, possibly due to activation of associated transcription factors, and (2) inactive forms are constituitively present in the membrane but lack the copper cofactor required for activity. Although the effects controlling the differential expression of the two forms of the enzyme are not clear, it appears that low concentrations of copper are sufficient to both repress sMMO expression and intensify pMMO expression. The expression of the soluble form can be achieved only at higher biomass, when copper concentration is insufficient for pMMO expression. This laboratory, as well as others, has found that copper is also required to maintain the activity of pMMO in vitro $(38,46,57)$. Cell lysis in a copper-deficient medium leads to dilution of the intracellular copper and rapid loss of pMMO activity. However, by adding copper, active pMMO is regenerated resulting in an increase in specific activity in vitro. Thus, the following equilibrium can be used to describe this phenomenon:

$$
\text { Holo-pMMO }-n \mathrm{Cu}^{2+} \text { (active) } \leftarrow \rightarrow \text { apo-pMMO (inactive) }+n \mathrm{Cu}^{++}
$$


Thus, the addition of excess copper ions to the cell extracts displaces the equilibrium to the left, resulting in the production of more active holoenzyme. Cook and Shiemke (1996) provided evidence in support of this hypothesis by using acetylene as a probe of the active site of pMMO (16). Increased concentrations of exogenous copper led to increasing amounts of radiolabeled $26 \mathrm{kDa}$ peptide after exposure to ${ }^{14} \mathrm{C}$-acetylene; suggesting copper ions activate pMMO by regenerating the active form of the enzyme.

Until recently, NADH has been the only reductant known to provide reducing equivalents to pMMO in vitro $(9,53)$. On the other hand, NADH cannot serve as a reductant in whole cell preparations, because it cannot cross the membrane. Formate, however, can cross the lipid membrane and provide reducing energy for pMMO. This suggests that pMMO may not receive its reducing equivalents directly from NADH in vivo. It has been suggested that such reducing equivalents may come from the oxidation of methanol by methanol dehydrogenase and that the electrons may be transferred along an electron transport chain in an indirect fashion to pMMO (9), although evidence for this is inconclusive.

\section{Project Overview}

A report from this laboratory recently demonstrated that pMMO could be reduced by quinols (16). In these experiments, pMMO was solubilized with detergent (lauryl maltoside) and an array of quinols was examined for their ability to reduce pMMO in the absence of NADH. It is surmised that the requirement of pMMO for quinols is responsible for previously reported purifications not being reproducible. If $\mathrm{pMMO}$ reduction by NADH were mediated by other proteins, then purification of pMMO would 
require that these components be present throughout the purification, as well. However, if quinones were used as a direct source of reducing equivalents, pMMO purification would require fewer protein components for reconstitution of activity. The latest published purification of pMMO seems to support this theory (Nguyen, H. (1997)) and this discussion will be elaborated by further work outlined in this dissertation.

Assuming that quinols serve to reduce pMMO directly, then it is necessary that an enzyme transfer reducing equivalents from $\mathrm{NADH}$ to the quinone prior to reduction of pMMO. Such an enzyme was recently purified by Cook (1997) and shown to be an NADH:quinone oxidoreductase (NDH) of type 2 (16). No evidence for the existence of a Complex I was observed, supporting the hypothesis that NADH reduces quinones via this type $2 \mathrm{NDH}$ and that these quinols may then reduce pMMO.

To investigate the respiratory chain of $M$. capsulatus and the apparent involvement of pMMO this document will concentrate on key areas yet to be studied. Our initial model suggests that reducing equivalents from $\mathrm{NADH}$ are transferred to the quinone pool via the type-2 NDH. This process is somewhat analogous to those occurring in many bacteria as well as in mitochondria $(3,4,7,10)$. Subsequently, the quinone pool would then partition between a putative $b c_{1}$-like complex $(10,15,28,59,60)$ and pMMO, capable of reducing either enzyme complex. The methanol produced by pMMO would then be oxidized by methanol dehydrogenase (20) and reducing equivalents from this reaction could be passed to a cytochrome $c$ (43) with subsequent reduction of a terminal oxidase $(8,11,13,35)$.

To study some of these interactions, the following areas were investigated. The quinone pool was extracted and analyzed in order to examine their ability to reduce 
pMMO. Additionally, the electron transfer chain was studied to determine how reducing equivalents partition between pMMO and either the putative $b c_{1}$-like complex or terminal oxidase(s). Bacteria as well as plants sometimes contain an alternative oxidase $(3,19,33,36,47)$, so this data would be instramental in elucidating the respiratory chain of this bacterium.

Thus, the overall goal of this project was to serve as a foundation for further research into the bioenergetics and respiratory chain of Methylococcus capsulatus (Bath). With prior work on mitochondrial, bacterial, and plant systems serving as a reference point, experiments within this project were performed in hopes that results would yield clues to the interplay of proteins/lipids within the respiratory chain. 


\section{REFERENCES}

1. Akent'eva, N.F., and R.I. Gvozdev. 1988. Purification and physiochemical properties of methane monooxygenase from membrane structures of Methylococcus capsulatus. Microbiology 53:91-96.

\section{Anderson, A.E., R. Bigidare, J.M. Brooks, J. Childress, C.R. Fisher, and}

M.C. Kennicut II. 1986. A methanotrophic marine molluscan (Bivavia, Mytilidae) synbiosis: mussels fueled by gas. Science 233: 1306-1308.

3. Anraku, Y. 1988. Bacterial electron transport chains. Ann. Rev. Biochem. 57:101-132.

4. Anraku, Y. and R.B. Gennis. 1987. The aerobic respiratory chain of Escherichia coli. Trends Biochem. Sci. 12:262-266.

5. Anthony, C. 1982. In The Biochemistry of Methylotrophs. Academic Press, London.

6. Anthony, C. 1986. Bacterial oxidation of methane and methanol. Adv. Microb. Physiol. 27:113-209.

7. Anthony, C.A. 1988. Bacterial Energy Transduction. Academic Press, London.

8. Babcock, G.T., and M. Wilkstrom. 1992. Oxygen activation and the conservation of energy in cell respiration. Nature. 356:301-309.

9. Bedard, C. and R. Knowles. 1989. Physiology, biochemistry and specific inhibitors of $\mathrm{CH}_{4}, \mathrm{NH}_{4}{ }^{+}$, and $\mathrm{CO}$ oxidation by methanotrophs and nitrifiers. Microbiol. Rev. 53:68-84.

10. Brand, M.D., and M.P. Murphy. 1987. Control of electron flux through the respiratory chain in mitochondria and cells. Biol. Rev. 62:141-193. 
11. Capaldi, R. 1990. Structure and function of cytochrome c oxidase. Annu. Rev. Biochem. 59:569-596.

12. Cardy, D.J.N., V. Laidler, G.P.C. Salmond, and J.C. Murrell. 1991. Molecular analysis of the membrane monooxygenase (MMO) gene cluster of Methylosinus trichosporium OB3b. Mol. Microbiol. 5:335-342.

13. Chan, S.I., and P.M. Li. 1990. Cytochrome $c$ oxidase: understanding nature's design of a proton pump. Biochemistry 29:1-12.

14. Chance, B. and G.R. Williams. 1956. The respiratory chain and oxidative phosphorylation. Adv. Enzymol. 17:65-134.

15. Colby, J., D.I. Stirling, and H. Dalton. 1977. The soluble methane monooxygenase of Methylococcus capsulatus (Bath): its ability to oxygenate nalkanes, n-alkenes, ethers, and alicyclic, aromatic and heterocyclic compounds. Biochem. J. 165:395-402.

16. Cook, S.A. and A.K. Shiemke. 1996. Evidence that copper is a required cofactor for the membrane-bound form of methane monooxygenase. J. Inorgan. Biochem. 63:273-284.

17. Davydov, A., R. Davydov, A. Graslund, J.D. Lipscomb, and K.K. Anderson. 1997. Radiolytic reduction of methane monooxygenase dinuclear iron cluster at 77K. J. Biol. Chem. 272:7022-7026.

18. DiSpirito, A.A., J. Gulledge, A.K. Shiemke, J.C. Murrell, M.E. Lidstrom, and C.L. Krema. 1992. Trichloroethylene oxidation by the membrane associated methane monooxygenase in type I, type II, and type X methanotrophs. Biodegradation 2:151-164. 
19. Douce, R. and M. Neuberger. 1989. The uniqueness of plant mitochondria. Annu. Rev. Plant. Physiol. 40:371-414.

20. Duine, J.A. 1991. Quinoproteins: enzymes containing the quinone cofactor pyrrolo-quinoline quinone, topaquinone or trytpophan tryptophan quinone. Eur. J. Biochem. 200:271-284.

21. Ensign, S.A., M.R. Hyman, and D.F. Arp. 1993. In vitro activation of ammonia monooxygenase from Nitrosomonas europaea by copper. J. Bacteriol. 175:1971-1980.

22. Fox, B.G., K.K. Surerus, E. Munck, and J.D. Lipscomb. 1988. Evidence for a $\mu$-oxo-bridged binuclear iron cluster in the hydroxylase component of the methane monooxygenase. J. Biol. Chem. 263:10553-10556.

23. Fox, B.G., W.A. Froland, J.E. Lipscomb, and J.D. Lipscomb. 1989. Methane monooxygenase from Methylosinus trichosporium OB3b: purification and properties of a three-component system with high specific activity. J. Biol. Chem. 264:10023-10033.

24. Fox, B.G., W.A. Froland, D.R. Jollie, and J.D. Lipsconb. 1990. Methane monooxygenase from Methylosinus trichosporium OB3b. Methods in Enzymol. 188:191-202.

25. Fox, B.G., Y. Liu, J.E. Dege, and J.D. Lipscomb. 1991. Complex formation between the protein components of methane monooxygenase from Methylosinus trichosporium OB3b. Identification of sites of component interaction. J. Biol. Chem. 266:540-550. 
26. Hanson, R.S. 1988. Ecology and diversity of methylotrophic organisms. Adv. Appl. Microbiol. 26:3-40.

27. Hanson, R.S., A.I. Netrusov, and K. Tsuji. 1991. In A. Balows, H.G. Truper, M. Dwqrkin, W. Hander, and K.H. Schleifer (eds) The Prokaryotes $2^{\text {nd }}$ ed. pp. 127. Springer-Verlag, New York.

28. Kagawa, Y., A. Kandrach, and E. Racker. 1973. Partial resolution of the enzymes catlysing oxidative phosphorylation. J. Biol. Chem. 248:676-684.

29. Kim, K. and S.J. Lippard. 1996. Structure and Mossbauer spectrum of a (m-1, 2-peroxo) bis (m-carboxylato) diiron (III) model for the peroxo intermediate in themethane monooxygenase hydroxylate reaction cycle. J. Am. Chem. Soc. 118:4914-4915.

30. Lee, S.K., J.C. Nesheim, and J.D. Lipscomb. 1993. Transient intermediates of the methane monooxygenase catalytic cycle. J. Biol. Chem. 268:21569-21577.

31. Lehninger, A.L., B. Reynafarje, A. Alexandre, and A. Villalobo. 1979. Proton stoichiometry and mechanisms in mitochondrial energy transduction. In Memebrane Bioenergetics (C.P. Lee, ed.). pp. 393-404. Addison-Wesley, London.

32. Lidstrom, M. 1991. In A. Balows, H.G. Truper, M. Dwqrkin, W. Hander, and K.H. Scleifer (eds) The Prokaryotes $2^{\text {nd }}$ ed. Springer-Verlag, New York.

33. Lin, E.C.C., and D.R. Kuritzkes. 1987. In F.G. Neidehart, J.L. Ingraham, K.B. Low, B. Magasanik, M. Schaechter, and H.E. Umberger (eds.). Escherichia coli and Salmonella typhimurium: Cellular and Molecular Biology. Am. Soc. Microbiol., Washington, D.C. 
34. Liu, Y., C. Nesheim, S.K. Lee, and J.D. Lipscomb. 1995. Gating effects of component $\mathrm{B}$ on oxygen activation by the methane monooxygenase hydroxylase component. J. Biol. Chem. 270:24662-24665.

35. Malmstrom, B.G. 1990. Cytochrome oxidase: some unsolved problems and controversial issues. Arch. Biochem. Biophys. 280:233-241.

36. Moore, A.L., and J.N. Siedow. 1991. The regulation and nature of the cyanideresistant alternative oxidase of plant mitochondria. Biochim. Biophys. Acta. 1059:121-140.

37. Murrell, J.C., and H. Dalton. 1983. Nitrogen fixation in obligate methanotrophs. J. Gen. Microbiol. 129:3481-3486.

38. Nguyen, H.T., A.K. Shiemke, S.J. Jacobs, B.J. Hales, M.E. Lidstrom and S.I. Chan. 1994. The nature of the copper ions in the membranes containing the particulate methane monooxygenase from Methylococcus capsulatus (Bath). J. Biol. Chem. 269:14995-15005.

39. Nielsen, A.K., K. Gerdes, H. Degn, and J.C. Murrell. 1996. Regulation of bacterial methane oxidation: transcription of the soluble methane monooxygenase operon of Methylococcus capsulatus (Bath) is repressed by copper ions. Microbiology 142:1289-1296.

40. Oldenhuis, R., J.L.R. Vink, D.B. Janssen, and B. Witholt. 1989. Degradation of chlorinated aliphatic hydrocarbons by Methylosinus trichosporium OB3b expressing soluble methane monooxygenase. Appl. Env. Microbiol. 55:28192826. 
41. Ortiz de Montellano, P.R., and P.R. Kunze. 1980. Self-catalyzed inactivation of hepatic cytochrome P-450 by ethnyl substrates. J. Biol. Chem. 255:28192826.

42. Paulsen, K.E., Y. Liu, B.G. Fox, J.D. Lipscomb, E. Munck, M.T. Stankovich. 1994. Oxidation-reduction potentials of the methane monooxygenase hydroxylase component from Methylosinus trichosporium OB3b. Biochemistry 33:713-722.

43. Pettigrew, G.W., and G.R. Moore. 1987. Cytochromes $c$ : Biological Aspects. Springer-Verlag, Heidelberg.

44. Poole, R.K., and W.J. Ingledew. 1987. In F.C. Neidehart, J.L. Ingraham, K.B. Low, B. Magasanik, M. Schaechter, and H.E. Umberger (eds.). Escherichia coli and Salmonella typhimurium: Cellular and Molecular Biology. Am. Soc. Microbiol., Washington, D.C.

45. Prior, S.D. and H. Dalton. 1985. Acetylene as a suicide substrate and activesite probe for methane monooxygenase from Methylococcus capsulatus. FEMS Microbiol. Lett. 29:105-109.

46. Prior, S.D. and H. Dalton. 1985. The effect of copper ions on membrane content and methane monooxygenase activity in methanol-grown cells of Methylococcus capsulatus (Bath). J. Gen. Microbiol. 131:155-164.

47. Puustinen, A., M. Finel, T. Haltia, R.B. Gennis, and M. Wlkstrom. 1991. Properties of the two terminal oxidases of Escherichia coli. Biochemistry 30:3936-3942. 
48. Rosenzweig, A.C., C.A. Frederick, S.J. Lippard, and P. Nordlund. 1993. Crystal structure of a bacterial non-haem iron hydroxylase that catalyses the biological oxidation of methane. Nature 366:537-543.

49. Rudd, J.W. and C.D. Taylor. 1980. Methane cycling in aquatic environments. Adv. Aquat. Microbiol. 2:77-150.

50. Semrau, J.D., A. Christoserdov, J. Lebron, A. Costello, J. Davagnino, E. Kenna, A.J. Holmes, R. Finch, J.C. Murrell, and M.E. Lidstrom. 1995. Particulate methane monooxygenase genes in methanotrophs. J. Bacteriol. 177:3071-3079.

51. Shiemke, A.K., S.A. Cook, T. Miley and P. Singleton. 1996. Detergent solubilization of membrane-bound methane monooxygenase requires plastoquinol analogs as electron donors. Arch. Biochem. Biophys. 321:421-428.

52. Shu, L., Nesheim, J.C., K. Kauffmann, E. Munck, J.D. Lipscomb, and L. Que. 1997. $\mathrm{An} \mathrm{Fe}_{2}{ }^{\mathrm{IV}} \mathrm{O}_{2}$ diamond core structure for the key intermediate of methane monooxygenase. Science 275:515-518.

53. Smith, D.D.S. and H. Dalton. 1989. Solubilization of methane monooxygenase from Methylococcus capsulatus (Bath). Eur. J. Biochem. 182:667-671.

54. Stainhorpe, A.C., J.C. Murrell, G.P. Salmond, H. Dalton, and V. Lees. 1989. Molecular analysis of methane monoxygenase from Mehtylococcus capsulatus (Bath). Arch. Microbiol. 152:154-159.

55. Stainthorpe, A.C., V. Lees, G.P. Salmond, H. Dalton, and J.C. Murrell. 1990. The methane monooxygenase gene cluster of Methylococcus capsulatus (Bath). Gene 91:27-34. 
56. Stainthorpe, A.C., G.P. Salmond, H. Dalton, and J.C. Murrell. 1991.

Screening obligate methanotrophs for soluble methane monooxygenase genes. FEMS Microbiol. Lett. 70:211-216.

57. Stanley, S.H., S.D. Prior, D.J. Leak, and H. Dalton. 1983. Copper stress underlies the fundamental change in intracellular location of methane monooxygenase in methane oxidizing organisms. Biotechnol. Lett. 5:487-492.

58. Tonge, G.M., D.E.F. Harrison, C.J. Knowles, and I.J. Higgins. 1975. Properties and partial purification of the methane-oxidizing enzyme system from Methylosinus trichosporium. FEBS Lett. 58:293-299.

59. Trumpower, B.L. 1990. The protonmotive Q cycle - energy transduction by couplingof proton translocation to electron transfer by the cytochrome $b c_{1}$ complex. J. Biol. Chem. 265:11409-11412.009

60. Trumpower, B.L. 1990. Cytochrome $b c_{1}$ complexes of microorganisms. Microbiol Rev. 54:101-129.

61. Tsien, H.C., G.A. Brusseau, R.S. Hanson, and L.P. Wackett. 1989. Biodegradation of trichloroethylene by Methylosinus trichosporium OB3b. Appl. Env. Microbiol. 55:3155-3161.

62. Weiss, H., T. Friedrich, G. Hofhaus, and D. Preis. 1991. The respiratorychain NADH dehydrogenase (complex I) of mitochondria. Eur. J. Biochem. 197:563-576.

63. Whittenbury, R. and H. Dalton. 1981. In M. Star et al. (eds) The Prokaryotes. pp. 894-902. Springer-Verlag, New York. 
64. Wilson, J.T. and B.H. Wilson. 1985. Biotransformation of trichloroethylene in soil. Appl. Environ. Microbiol. 49:242-243.

65. Woodland, M.P. and H. Dalton. 1984. Purification and characterization of component A of the methane monooxygenase from Methylococcus capsulatus (Bath). J. Biol. Chem. 259:53-60.

66. Zahn, J.A. and A.A. DiSpirito. 1996. Membrane-associated methane monooxygenase from Methylococcus capsulatus (Bath). J. Bacteriol. 178:10181029. 


\section{CHAPTER TWO}

Methods and Materials 


\section{Bacterial Growth (Methanotrophs)}

Methylococcus capsulatus (Bath) were grown in Nitrate Salts Medium (NMS) as described by Dalton and Whittenbury (1981). Phosphate buffer (pH 6.8), Staley's vitamins (5), and $\mathrm{CuSO}_{4}$ at varying concentrations were also added. To some cultures, iron (both in the +2 and +3 oxidation states) was added as described by DiSpirito, et al. (1996). M. capsulatus were maintained on $1.8 \%$ agar plates containing $2 \mu \mathrm{M} \mathrm{CuSO}_{4}$ at $37^{\circ} \mathrm{C}$ in a $20 \%$ methane, $80 \%$ air environment. Large-scale growth was initiated in 250 mL flasks containing $50 \mathrm{~mL}$ NMS with $2 \mu \mathrm{M} \mathrm{CuSO}_{4}$. These cultures were shaken at $45^{\circ} \mathrm{C}$ for approximately two days and supplemented with methane in air daily. Each culture was transferred to a 2-L flask containing 500mL NMS, $10 \mu \mathrm{M} \mathrm{CuSO}_{4}$, and iron, and maintained under the same conditions for two days. After this period, the cultures (typically 2 liters total) were used to inoculate nine liters of NMS containing $20 \mu \mathrm{M}$ $\mathrm{CuSO}_{4}$ in a 14-L fermentor (New Brunswick Scientific) with the addition of 10 to $20 \mu \mathrm{M}$ ferric and ferrous salts. The fermentor was maintained at $45^{\circ} \mathrm{C}$ with continual stirring and sparged with $20 \%$ methane in air, at a flow rate of approximately $2 \mathrm{SCFH}$. Following a two to three day incubation period, the cells were harvested via centrifugation at 4000xg, and the bacterial pellet was washed once and resuspended in 150mL 10mM PIPES, pH 7.0 (Fisher, Enzyme Grade). This cell suspension was stored in a septum-sealed vial at $4^{\circ} \mathrm{C}$ under an argon atmosphere until used.

\section{Cell Lysis and Membrane Isolation}

Bacteria were lysed by passing an aliquot of the cell suspension through a French Pressure cell (SLM Aminco) at 18,000-20,000 psi. Prior to lysis, cells were supplemented with DNase I (Sigma, $40 \mu \mathrm{g} / \mathrm{mL}$ ). Varying concentrations of $\mathrm{CuSO}_{4}$ were 
added when pMMO activity was to be measured, but copper was omitted when oxidase activity was investigated. The cell lysate was centrifuged at $31,000 \mathrm{x} g$ for $5-8$ minutes to remove cell debris and unbroken cells, and then centrifuged at 106,000x $\mathrm{g}$ for 90' to pellet the membrane. The membrane pellet was resuspended in 10mM PIPES, pH 7.0 with a Dounce homogenizer. Membrane suspensions used for pMMO activity were again supplemented with $\mathrm{CuSO}_{4}$, whereas membranes used for oxidase activity and spectroscopic kinetics were not.

\section{Enzymatic Activity Assays (pMMO Membrane Samples)}

The pMMO activity was measured according to the previously published propene-oxidation assay (2) at room temperature (except for Chapter IV, where $45^{\circ} \mathrm{C}$ was used). The assay was initiated by addition of $1 \mathrm{~mL}$ propene to $6-\mathrm{mL}$ sealed serum vials containing the particulate fraction, 5mM NADH (Sigma), 10mM PIPES (pH, 7.0, Enzyme Grade, Fisher), and variable concentrations of $\mathrm{CuSO}_{4}$ (added exogenously). The liquid volume was held constant at $1.0 \mathrm{~mL}$. The NADH was lyophilized such that the ethanol (present as a contaminant) was removed before stock solutions were prepared. In assays employing whole cells, $5 \mathrm{mM}$ formate (sodium salt) was utilized as the source of reducing equivalents (1). The concentration of propene oxide produced by pMMO was measured by gas chromotography (SRI 8610 GC, equipped with an FID). Enzyme activity, in nmol/min, was determined from the slope of the linear portion of a graph of propene oxide concentration versus time. To calculate specific activity, the protein concentrations were measured using the bicinchoninic acid assay (Pierce Biochemical) (6) and bovine serum albumin (Sigma) as the standard. Membrane suspensions were 
typically assayed at concentrations of 1.25 to $2.25 \mathrm{mg} / \mathrm{mL}$ total protein, while whole cell suspensions were typically assayed at concentrations of 3.75 to $10 \mathrm{mg} / \mathrm{mL}$ total protein.

\section{Detergent-solubilized pMMO Activity Assays}

Enzymatic activity for the detergent-solubilized pMMO was analyzed as mentioned above, except that quinones plus NADH, or quinols (without NADH present) were added as the reducing system. Both quinones and quinols were added as solids to prevent interference from organic solvents (i.e., methanol, ethanol, and DMSO) in the gas chromatogram. A volume of stock solution containing 0.1 to $10 \mu$ moles of quinone (or quinol) was delivered to the sealed serum vial, and the volatile organic solvent was subsequently removed by heating to $55^{\circ} \mathrm{C}$ under vacuum. The vial was then opened to allow delivery of the sample components. The vial was then sealed and $1 \mathrm{~mL}$ of propene was added to initiate the reaction. The concentration of propene oxide was measured as described above.

\section{Preparation of Quinols}

2,3-Dimethylhydroquinol, trimethylhydoquinol, and menaquinone were purchased from Aldrich Chemical Company while tetramethyl-p-benzoquinone (duroquinone), 2,3-dimethoxy-5-methyl-6-decyl-1,4-benzoquinone (decyl-ubiquinone), and decyl-plastoquinone were purchased from Sigma Chemical Company. All six quinones were used without further purification. Quinones were reduced by the method of Rich (5), or by small additions of dithionite/sodium borohydride followed by extraction $(3 \mathrm{X})$ into an ether layer in a small test tube or acidification/precipitation by 
addition of $1 \mathrm{~N} \mathrm{HCl}$. The quinols were stored in acidified ethanol in a septum-sealed serum vial under an argon atmosphere, at -20 to $-70^{\circ} \mathrm{C}$. Concentrations of the quinol solutions were determined based on their absorbances in conjunction with applicable absorbtivity coefficients (decyl-plastoquinol, $\varepsilon 290=3450 \mathrm{M}^{-1} \mathrm{~cm}^{-1}$; duroquinol, $\varepsilon 283$ $=2140 \mathrm{M}^{-1} \mathrm{~cm}^{-1}$; decyl-ubiquinol, $\varepsilon_{283}=4100 \mathrm{M}^{-1} \mathrm{~cm}^{-1}$; trimethylquinol, $\varepsilon_{290}=2050 \mathrm{M}^{-}$ ${ }^{1} \mathrm{~cm}^{-1} ;$ 2,3-dimethylquinol, $\varepsilon_{300}=3980 \mathrm{M}^{-1} \mathrm{~cm}^{-1}$; menaquinol, $\varepsilon_{330}=3100 \mathrm{M}^{-1} \mathrm{~cm}^{-1}, 18^{\prime}-$ methylene $\mathrm{CoQ}_{8}, \varepsilon_{280}=3850 \mathrm{M}^{-1} \mathrm{~cm}^{-1}$ (from this work)).

\section{Octanol-water Partition Coefficients of Exogenous Quinols}

An amount of the solid quinol was dissolved in octanol to give a maximal absorbance of 0.7 to 0.9 . A $25-\mathrm{mL}$ aliqout of the octanol solution of the quinol was shaken with $25 \mathrm{~mL}$ of water (acidified with $3 \mathrm{mN} \mathrm{HCl}$ ), in a separatory funnel for 15 minutes. The octanol layer was drained into a beaker containing a few grams of $\mathrm{Na}_{2} \mathrm{SO}_{4}$ to remove water. The partition coefficient was defined as the proportion of the quinol that remained in octanol after equilibrating with water. This was determined from the UV absorbance of the octanol layer before and after shaking with water.

\section{pMMO Detergent Solubilization}

Detergents were prepared as stock solutions containing 10 or $20 \%$ detergent (w/v) in 10mM PIPES, pH 7.0. The detergent stock solution was added with stirring (or vortexing) to membrane suspensions containing $5-45 \mathrm{mg} / \mathrm{mL}$ of total protein. This detergent/membrane suspension was vortexed at room temperature or at $3^{\circ} \mathrm{C}$ for 15 to 45 minutes (variation of the time and temperature had no significant effect on pMMO activity of the solubilized fraction). The suspension was then centrifuged at $106,000 \mathrm{x} g$ for 60 to 90 minutes to separate the detergent-solubilized proteins from the insoluble 
pellet. The soluble fraction was used without further manipulations. For assays of the insoluble fraction, the pellet was resuspended in the original volume of $10 \mathrm{mM}$ PIPES buffer, pH 7.0 using a Dounce homogenizer. Lauryl maltoside was obtained from Calbiochem or Anatrace and used without further purification. Other detergents were obtained from Boehringer-Mannheim.

\section{Acetylene Labeling of pMMO}

In a septum-sealed vial, $\sim 500 \mu \mathrm{L}$ of detergent-solubilized membrane sample was incubated with $75 \mu \mathrm{L}$ of $\left[{ }^{14} \mathrm{C}\right]$-acetylene $(60 \mathrm{mCi} / \mathrm{mmol}$; New England Nuclear) in air, for at least 30 minutes (previous time course experiments indicated that all of the available acetylene binding sites were occupied in $<30$ minutes). Samples were evacuated and flushed with $1 \mathrm{~mL}$ of unlabeled acetylene to remove non-specifically bound radioactivity, incubated for 5 minutes at room temperature, evacuated, and flushed with acetylene again. Radioactivity was measured by scintillation counting of $150 \mu \mathrm{L}$ of the sample in $3 \mathrm{~mL}$ of Dimiscint for 5 minutes on a Beckman LS 5000TD scintillation counter. Aliquots of the same $\left[{ }^{14} \mathrm{C}\right]$-treated samples were electrophoresed as described below. Radiolabeled bands were detected using a Molecular Dynamics phosphorimager, with ImageQuant software.

\section{Gel Electrophoresis}

SDS-PAGE was performed according to the method of Laemmli (3). Radiolabeled samples were diluted fourfold with a 1:1 mixture of sample dye (50\% glycerol; $0.01 \%$ bromophenol blue) and sample buffer (0.25M Tris, $\mathrm{pH} 6.8 ; 8 \%$ SDS). An appropriate volume $(\sim 50 \mu \mathrm{L})$ of the prepared samples was loaded onto the lanes of a $15 \%$ discontinuous polyacrylamide gel with a $4.5 \%$ stacking gel. Molecular weight 
markers (Sigma) were loaded in adjacent lanes to calibrate the molecular weight of the proteins. Samples were electrophoresed at a constant current of $30 \mathrm{~mA}$ until complete ( 4-5 hours). The gels were fixed (50\% methanol; $10 \%$ glacial acetic acid) for 30 minutes, stained for $\sim 2$ hours with $0.16 \%$ Coomassie blue G-250 (Inprotech). And destained overnight (10\% methanol; $12 \%$ glacial acetic acid).

\section{Oxygen Uptake Studies}

The rate of oxygen uptake was measured in both membrane fractions and whole cell suspensions, using a YSI polarographic oxygen electrode equipped with a constant temperature circulating water bath. All experiments were performed at $42^{\circ} \mathrm{C}$. The sample chamber contained $3.0 \mathrm{~mL}$ of the sample suspension in $10 \mathrm{mM}$ PIPES (pH 7.0). After an initial incubation of the sample buffer (typically 10 minutes), the probe was lowered into the chamber and the probe/membrane allowed to come to temperature. Excessive noise in the baseline was eliminated by overnight incubation of the probe in a dilute sample of membrane or whole cell suspension to reduce static electricity. After attaining a relatively stable baseline, the appropriate sample was delivered into the sample chamber using a Hamilton syringe. After the oxygen uptake curve reached a linear stage, substrate was added to increase respiration above basal levels. If inhibition studies were performed, the appropriate inhibitor was added after the substrate-stimulated rate was linear. The following inhibitors were used: piericidin A (a gift from Dr. Thorsten Friedrich), amytal (Eli Lilly Company), rhein (Sigma), HQNO (Sigma), caffeine (Sigma), myxothiazole (Sigma), antimycin A (Sigma), SHAM; Salicylhydroxamic acid (Sigma), cyanide (Fisher Scientific), octyl gallate (Sigma). 


\section{Isolation, Purification and Characterization of Endogenous Quinones}

A single culture of pMMO-expressing Methylococcus capsulatus was used for this study. The cells were grown, harvested, and lysed as mentioned above. The pelleted membrane material was lyophilized for 48 hours then placed into a 1-L beaker to which $500 \mathrm{~mL}$ of $n$-pentane (Fisher Scientific) was added. The suspension was stirred for 5 hours to extract the hydrophobic quinones. The suspension was gravity filtered through magnesium sulfate (Fischer Scientific) in analytical grade Whatman filter paper placed in a glass funnel. The filtrate (approximately $350 \mathrm{~mL}$ ) was filtered once again through analytical grade filter paper to remove any debris which had escaped the first pass. The canary yellow extract was then examined via UV-visible spectrophotometry from 200 to 400nm utilizing an SLM Aminco DW 2000 spectrophotometer for the broad 280nm absorbance characteristic of quinones. The extract was dried to a viscous oil and dissolved in HPLC grade methanol (Fisher Scientific) to a volume of approximately 120$\mathrm{mL}$. BHT was added to inhibit oxidation. The extract was chromatographed using a Waters Millennium HPLC system interfaced with a Waters 996 Photodiode array detector. The quinones were eluted from a Waters reversed-phase $\mathrm{C}_{18}$ column ( 250 x 4.6 $\mathrm{mm})$ under an isocratic system $(1.0 \mathrm{ml} / \mathrm{min})$ with pure methanol (HPLC grade) as the mobile phase. No pre-column or in-line filters were used. The quinones were detected at a wavelength of $280 \mathrm{~nm}$. To aid in identification, one of the purified analytes was mixed with a solution of quinone standards $\left(\mathrm{Q}_{6}, \mathrm{Q}_{7}, \mathrm{Q}_{9}\right.$, and $\mathrm{Q}_{10}$; Sigma). This mixture was chromatographed as above, with a flow rate of $0.5 \mathrm{~mL} / \mathrm{min}$. All integration and data manipulation was performed with the Waters Millennium software. 
GC/MS were recorded using a Finnigan Model 4500 mass spectrometer interfaced with a Varian Model 3400 gas chromatograph containing a DB-5 column using a direct insertion probe. Ionizing voltage ranged from $18 \mathrm{eV}$ to $70 \mathrm{eV}$ with a temperature range of 50 to $300^{\circ} \mathrm{C}$ at 60 degrees/minute. Data manipulation was performed using an Incos data system. ${ }^{1} \mathrm{H}$ and ${ }^{13} \mathrm{C}$ NMR spectra were recorded on a Varian $300 \mathrm{MHz}$ FT-NMR. Nuclear magnetic resonance samples were dissolved in deuterated chloroform.

\section{Spectroscopic Kinetic Studies}

The reduction of cytochrome $c$ was followed as a function of time at a wavelength of $555 \mathrm{~nm}$. This was performed on an SLM Aminco DW-2000 UV-visible spectrophotometer equipped with a stirring apparatus. Samples were maintained at $42^{\circ} \mathrm{C}$ with the aid of a constant temperature bath and water circulation attachment surrounding the sample cuvette(s). Reduction experiments were performed on pMMO-expressing $M$. capsulatus membrane preparations (suspended in 10mM PIPES, $\mathrm{pH}$ 7.0) with methanol (Fisher Scientific) as the source of reducing equivalents. To inhibit flow of reducing equivalents, octyl gallate (Sigma) and potassium cyanide (Fisher Scientific) were utilized, dissolved in methanol (or DMSO) and water, respectively. For changes in signal amplitude, exogenous cytochrome $c$ (horse heart, Sigma) was added to the sample cuvette from a stock solution of the protein dissolved in 10mM PIPES, $\mathrm{pH}, 7$. 


\section{REFERENCES}

1. Anthony, C. 1986. Bacterial oxidation of methane and methanol. Adv. Microb. Physiol. 27:113-209.

2. Colby, J., D.I. Stirling, and H. Dalton. 1977. The soluble methane monooxygenase of Methylococcus capsulatus (Bath): its ability to oxygenate $n$ alkanes, $n$-alkenes, ethers, and alicyclic, aromatic, and heterocyclic compounds. Biochem. J. 165:395-402.

3. Laemmli, U.K. 1970. Cleavage of structural proteins during the assembly of the head of bacteriophage T4. Nature. 227:680-685.

4. Lidstrom, M. 1988. Isolation and characterization of marine methanotrophs. Antoine van Leewenhoek J. Microbiol. Serol. 54:189-199.

5. Rich. P.R. 1981. Electron transfer reactions between quinols and quinones in aqueous and aprotic media. Biochim. Biophys. Acta. 637:28-33.

6. Smith, P.K., R.I. Krohn, G.T. Hermanson, A.K. Mallia, F.H. Gartner, M.D. Provenzano, E.K. Fujimoto, N.M. Goeke, B.J. Olson, and D.C. Klenk. 1985. Measurement of protein using bicinchoninic acid. Anal. Biochem. 150:76-85. 


\section{CHAPTER THREE}

\section{Detergent Solubilization of membrane-bound Methane}

\section{Monooxygenase Requires Plastoquinol Analogs as Electron Donors}

Andrew K. Shiemke, Scott A. Cook, Tim Miley, and Patrick Singleton

Published in the Archives of Biochemistry and Biophysics. 1995. 321: 421-428 


\begin{abstract}
Quinols can provide reducing equivalents for the membrane-bound methane monooxygenase (pMMO), substituting for $\mathrm{NADH}$ in whole cells and membranes. Furthermore, quinols are effective reductants for the detergent-solubilized enzyme, whereas NADH is ineffective. The decyl analog of plastoquinol and duroquinol $(2,3,5,6-$ tetramethylbenzoquinol) provide the greatest methane monooxygenase activity in whole cells and membrane suspensions, as well as detergent-solubilized samples. Lauryl maltoside is by far the best detergent for solubilization of catalytically active methane monooxygenase. Optimal pMMO activity in the detergent-solubilized fraction is obtained with a ratio of $\sim 1.7$ milligram of detergent per milligram of membrane protein, independent of protein concentration. The detergent-solubilized pMMO retains its sensitivity to inhibition by cyanide, acetylene, and EDTA. It is also stimulated by exogenous copper, as in isolated membrane fractions. Reaction of the detergentsolubilized enzyme with $\left[{ }^{14} \mathrm{C}\right]$ acetylene results in labeling of the 26-kDa peptide, analogous to the behavior observed for isolated for isolated membrane suspensions. The selectivity of pMMO for duroquinol and decyl-plastoquinol, relative to other structurally similar quinols suggests that the enzyme obtains reducing equivalents directly from a quinol (probably plastoquinol) in vivo.
\end{abstract}




\section{INTRODUCTION}

Methane monooxygenase (MMO) is the $\mathrm{O}_{2}$-dependent enzyme that initiates methane oxidation in methanotrophic bacteria (3). The enzyme also requires reducing equivalents, presumably to activate $\mathrm{O}_{2}$. As is typical of mixed-function oxygenases (15), the oxygen molecule is split during catalysis, with one oxygen atom reduced to water, and the other oxygen atom incorporated into methane, producing methanol (1). Methanol is subsequently oxidized to formaldehyde by methanol dehydrogenase, generating reduced cytochrome $c$ (11). Formaldehyde can be assimilated to provide carbon, or further oxidized to carbon dioxide, thereby providing energy for the organism (2).

MMO is critical to the global carbon cycle, initiating the major route for methane produced by anaerobic fermentation to re-enter the biosphere (12). Knowledge of the enzymatic mechanism of MMO may aid in the design of catalysts for the large-scale oxidation of methane. Such catalysts are crucial to the more effective use of methane as a fuel and industrial feedstock (17). In addition, MMO is one of the few enzymes able to oxidize small, unactivated alkanes and halogenated hydrocarbon solvents (9), such as trichloroethylene (others include ammonia monooxygenase (4), propane monooxygenase (32), and toluene dioxygenase (31)). Hence, methanotrophic bacteria (and MMO) are the focus of many investigations utilizing bioremediation to remove such solvents from groundwater (23).

Two distinct forms of methane monooxygenase are known. The soluble form (sMMO) is a cytosolic, multi-component enzyme that obtains reducing equivalents directly from NADH. It has been purified to homogeneity, and a high-resolution crystal structure has recently been published (21). The hydroxylase component of sMMO 
contains two dinuclear-iron active sites. The membrane bound form of MMO (referred to as pMMO, since it is localized in the particulate fraction) has not been well characterized. Previous reports of the purification of pMMO $(29,1)$ are not reproducible $(3)$, hence little is known regarding its structure and mechanism. In contrast with sMMO, copper is required for pMMO activity $(18,16)$. This copper appears to be loosely bound, as pMMO is inhibited by metal chelators such as EDTA, and dithiocarbamates, which have no effect on sMMO $(22,28)$. The two forms of MMO also differ in substrate specificity: sMMO can oxidize large aliphatic and aromatic compounds, whereas pMMO cannot oxidize hydrocarbons larger than butane (3, 5). Although several species of methanotroph are able to express both pMMO and sMMO, they are not expressed concurrently, and are the products of separate genes $(26,27)$. The ammonia monooxygenase (AMO) found in ammonia-oxidizing bacteria is similar to pMMO in all of the aspects described above (10). AMO has not yet been purified and also remains poorly characterized.

Progress in purification and characterization of pMMO has been slowed by the lability of the enzyme in vitro, and the lack of methods for preparing active, detergentsolubilized pMMO. The problem of pMMO instability has recently been resolved: it results primarily from the disociation of weakly-bound copper (16). The enzyme is stabilized in vitro by excess copper. Solubilization of active pMMO remains the major obstacle to purification of this enzyme. Although Dalton and coworkers reported solubilization of pMMO with lauryl-maltoside, activation of the enzyme required reconstitution with phospholipids, and the reconstituted preparations were inactivated by 
fractionation (25). Solubilization with other detergents resulted in complete inactivation that could not be reversed by reconstitution with phospholipids (25).

The results of Smith and Dalton (25) could arise from detergent-induced disruption of an electron-transport chain from NADH to pMMO. In contrast, a reductant that directly reduces pMMO should be able to support activity after detergent solubilization. To test this theory, we have examined a wide range of reducing agents for their ability to donate electrons to pMMO. Our results suggest that quinols are capable of directly reducing membrane-bound and detergent-solubilized pMMO. The enzyme is selective for specific quinols, suggesting that the reductant in vivo is also a quinol. 


\section{RESULTS}

\section{Reduction of Membrane-bound MMO by Quinols}

As is true of all monooxygenase enzymes, pMMO requires reducing equivalents for activity. In this report we show that certain quinols can replace NADH as the source of reducing equivalents for pMMO from $M$. capsulatus. The activity of pMMO in whole cells and membranes is significantly greater with decyl-plastoquinol and duroquinol despite their structural similarity to the less effective quinols (Fig. 1). Duroquinol and decyl-plastoquinol were not as effective as $\mathrm{NADH}$ however, perhaps due to the low solubility of the quinols in aqueous solution. The pMMO activity of the membrane suspensions was proportional to duroquinol concentration, and was saturated at $\sim 2 \mathrm{mM}$ duroquinol (data not shown). Other reductants that are effective with many monooxygenases and oxidases were also examined for their ability to reduce pMMO, including dithionite, ascorbate, methyl viologen, benzyl viologen, and ascorbate/TMPD. In no case was pMMO activity observed in membranes treated with these reductants.

\section{Detergent-solubilization of pMMO}

Addition of lauryl maltoside to membrane suspensions enhanced the pMMO activity with all of these quinol reductants, especially decyl-plastoquinol (data not shown). No pMMO activity with $\mathrm{NADH}$ as the reductant was observed after solubilization. This observation appears to result from disruption of electron transfer from NADH to pMMO, since the solubilized enzyme remained active with duroquinol as the reductant, as demonstrated in Fig. 2. In this experiment, membranes were treated with increasing concentrations of lauryl-maltoside, and the soluble and pellet fractions were assayed separately for pMMO activity, using either NADH or duroquinol as the 
<smiles>Cc1c(C)c(C)c(O)c(O)c1C</smiles><smiles>CCCCCCCCCCc1cc(O)c(C)c(C)c1O</smiles>

Decyl-Plastoquinol<smiles>COc1c(O)cc(C)c(O)c1OC</smiles>

Coenzyme $Q_{0}$<smiles>CCCCCCCCCCc1c(C)c(O)c(OC)c(OC)c1O</smiles>

Decyl-Ubiquinol<smiles>Cc1cc(O)c2ccccc2c1O</smiles><smiles>Cc1cc(O)c(C)c(C)c1O</smiles>

Trimethylquinol<smiles>Cc1c(O)ccc(O)c1C</smiles>

2,3-Dimethylquinol

Figure 1

Structures of the quinol reductants used to assay pMMO activity. 

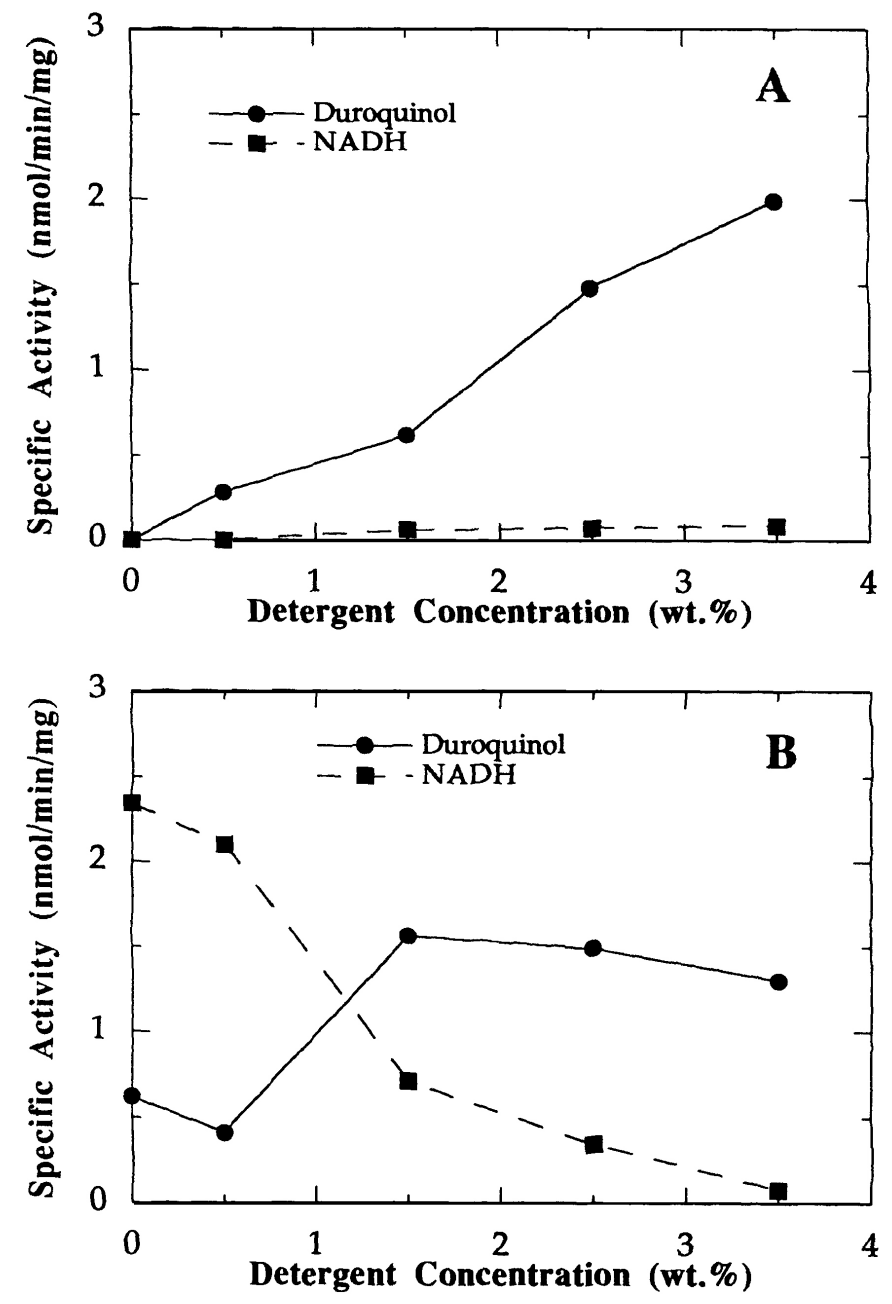

Figure 2

Increased detergent concentration shifts pMMO activity from the membrane to the soluble fraction. The activity of pMMO in the detergent-solubilized fraction (A) and insoluble fraction (B), are shown as a function of lauryl-maltoside concentration with duroquinol $(\bullet)$ or NADH $(\bullet)$ as reductant. 
electron donor. With duroquinol, the pMMO activity of the soluble fraction was proportional to detergent concentration (Fig. 2A). In contrast, NADH-dependent pMMO activity was observed exclusively in the pellet, and was inversely proportional to detergent concentration (Fig. 2B). Duroquinol-dependent pMMO activity in the pellet was also inversely proportional to detergent at concentrations greater than $1.5 \%$ (Fig. 2B). The stimulatory effect of lauryl-maltoside is responsible for the initial increase in the duroquinol-dependent activity of the pellet (Fig. 2B). No pMMO activity was observed in the detergent-solubilized fraction in the presence of dithionite, ascorbate, or methyl viologen. Thus, pMMO remained active after solubilization, but only with specific quinol reductants.

Fig. 3 shows that a detergent-to-protein weight ratio of $\sim 1.7$ was optimal for solubilizing active pMMO, and activity decreased markedly when the detergent concentration increased further (Fig. 3). The efficiency of solubilization was independent of protein concentration: maximum activity was observed at the same detergent-toprotein ratio for membranes suspensions containing $7 \mathrm{mg} \mathrm{mL}^{-1}$ and $20 \mathrm{mg} \mathrm{mL}^{-1}$ total protein (Fig 3). The amount of protein in the soluble fraction increased similarly, also reaching a maximum at $\sim 75 \%$ of the total membrane protein with a detergent-to-protein ratio of $\sim 1.7$ (Fig. 3). For reasons that remain unclear, solubilization of highly active pMMO from $M$. capsulatus membranes was possible only with lauryl-maltoside. No pMMO activity was observed with duroquinol or $\mathrm{NADH}$, in either the supernatant or pellet, after solubilization with Triton X-100, Tween-20, zwittergent 3-12, nonidet P40, or synperonic. Although some pMMO activity was observed after solubilization with 


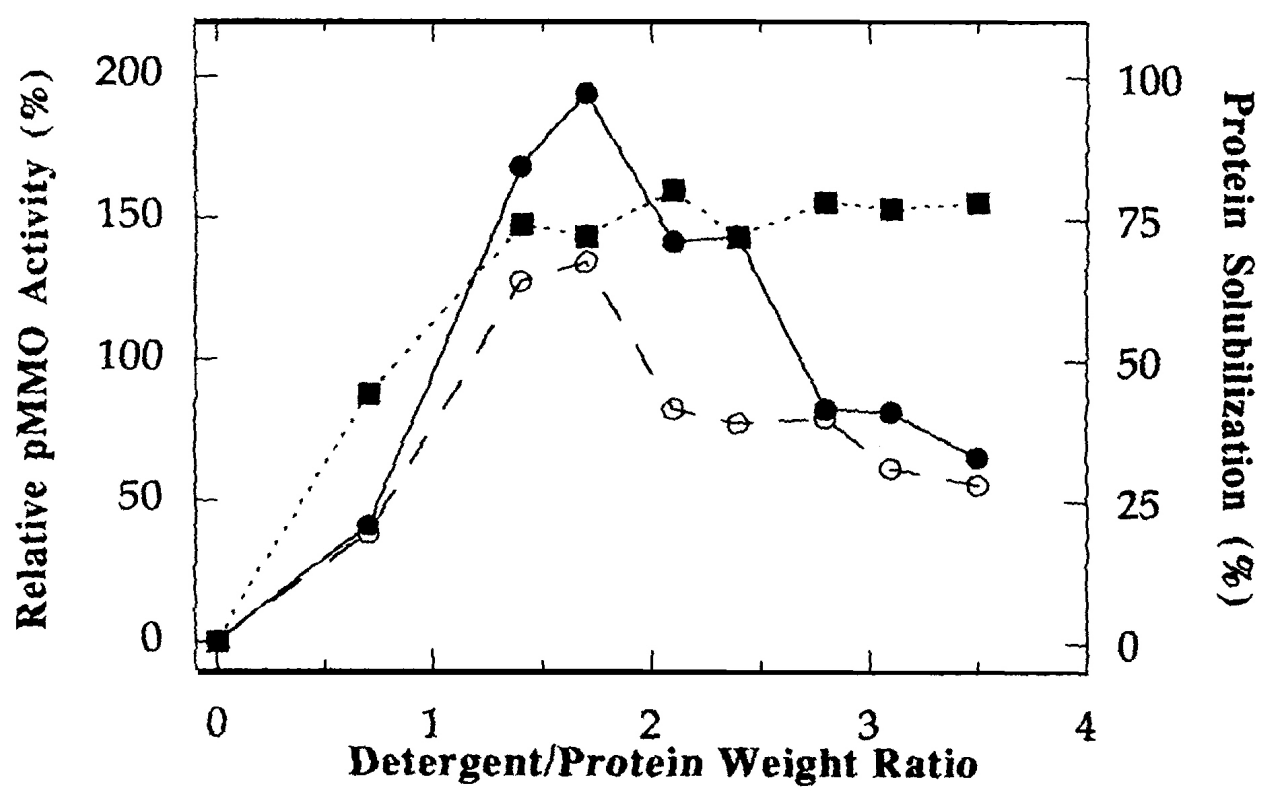

Figure 3

Solubilization of pMMO from the membranes as a function of lauryl-maltoside-to-protein weight ratio. Membranes were solubilized at a protein concentration of $7 \mathrm{mg} \mathrm{mL}^{-1}$ (o), or $20 \mathrm{mg} \mathrm{mL}^{-1}(\bullet)$. The specific activity of pMMO in the soluble fraction was determined in the presence of $15 \mathrm{mM}$ duroquinol and is reported relative to the specific activity of the membrane fraction assayed with duroquinol $\left(0.7 \mathrm{nmol} \mathrm{min} \mathrm{mL}^{-1}\right)$. The percentage of total protein solubilized is shown for the $20 \mathrm{mg} \mathrm{mL}^{-1}$ sample $(\diamond)$. Similar results were obtained at $7 \mathrm{mg} \mathrm{mL}^{-1}$. 
diheptanoyl-phosphatidylcholine, it was $<10 \%$ of the activity observed with laurylmaltoside (data not shown).

The relative activities of the quinols with detergent-solubilized pMMO is similar to that observed with whole cells and membranes (Table I), indicating the enzymatic mechanism is unaffected by solubilization. Detergent-solubilized pMMO exhibited stimulation by copper and inhibition by cyanide, EDTA, and acetylene (Table II), as does the membrane-bound enzyme $(3,16)$, providing additional evidence that the catalytic core is not altered by solubilization. Finally, suicide inhibition of pMMO by acetylene was also unaffected by solubilization. Treatment of the detergent-soluble fraction with $\left[\mathrm{C}^{14}\right]$ acetylene, $\mathrm{O}_{2}$, and duroquinol results in irreversible inhibition of pMMO (Table II), and irreversible binding of the radiolabel (Fig. 4). Just as with membrane-bound pMMO, the irreversible binding of acetylene to the detergent-solubilized enzyme requires a reductant (duroquinol in this case), and is prevented by the pMMO inhibitor EDTA, and by protein denaturation (Fig. 4). Electrophoretic analysis of the solubilized fraction after exposure to $\left[\mathrm{C}^{14}\right]$ acetylene and duroquinol shows the radiolabel bound primarily to a 26 $\mathrm{kDa}$ protein, a significantly weaker radiolabeled band is observed with an apparent molecular weight of $\sim 50 \mathrm{kDa}$ (Fig. 4, inset). This band may result from aggregation of the $26 \mathrm{kDa}$ protein, as previously reported for pMMO from M. capsulatus (9). The low molecular weight radiolabeled band in Figure 4 ran near the dye front and may be due to degradation of the $26 \mathrm{kDa}$ protein or reaction of oxidized acetylene with buffer, detergent, or phospholipid. 
$\underline{\text { Table } 1 \text { Comparison of pMMO activity with various substituted para-benzoquinols. }}$

$$
\text { pMMO Specific Activity (nmol min } \mathrm{mg}^{-1} \text { ) }
$$

\section{Soluble Redox}

\begin{tabular}{|c|c|c|c|c|c|}
\hline Reductant & hole Cells & Membranes & Fraction $^{\mathrm{b}}$ & Potential $^{\mathrm{c}}$ & Hydrophobicity $^{\mathrm{d}}$ \\
\hline $\mathrm{NADH}$ & $24.2^{\mathrm{e}}$ & 6.2 & $<0.1$ & $-340 \mathrm{mV}$ & n.d. ${ }^{\mathrm{f}}$ \\
\hline Decyl-plastoquinol & 4.44 & 0.38 & 2.70 & $30 \mathrm{mV}$ & $97.9 \pm 0.008$ \\
\hline Duroquinol & 2.90 & 1.56 & 1.46 & $50 \mathrm{mV}$ & $96.7 \pm 0.005$ \\
\hline Decyl-Ubiquinol & 0.41 & 0.18 & 0.36 & $40 \mathrm{mV}$ & $94.6 \pm 0.005$ \\
\hline Coenzyme $\mathrm{Q}_{0}$ & 1.22 & 0.08 & 0.18 & & $90.4 \pm 0.02$ \\
\hline Menaquinol & 0.06 & $<0.05$ & 0.06 & $0 \mathrm{mV}$ & $96.8 \pm 0.008$ \\
\hline 2,3-Dimethyl Quinol & $<0.05$ & n.d. & $<0.05$ & $210 \mathrm{mV}$ & $96.3 \pm 0.002$ \\
\hline Trimethyl Quinol & 0.12 & n.d. & 0.07 & $115 \mathrm{mV}$ & $96.4 \pm 0.006$ \\
\hline
\end{tabular}

${ }^{\mathrm{a}}$ Reductants were present at concentrations of $5 \mathrm{mM}$ for whole cells and membranes, $10 \mathrm{mM}$ for the soluble fraction. Structures of the quinones are shown in Figure 1.

${ }^{b}$ Membranes were solubilized with a detergent:protein weight ratio of 1.7, with lauryl maltoside. ${ }^{c} \mathrm{E}^{0}$ values from reference $8 .{ }^{\mathrm{d}}$ The octanol-water partition coefficients for the quinols determined at room temperature. Values represent the average of 6-10 separate determinations. ${ }^{\mathrm{e}}$ Activity assayed with 5mM Formate. ${ }^{\mathrm{f}}$ nd, not determined. 
Table 2 Effect of Inhibitors and $\mathrm{Cu}(\mathrm{II})$ on Solubilized $\mathrm{pMMO}^{\mathrm{a}}$.

Addition $\quad$ pMMO Activity (\% of Control)

$\begin{array}{ll}\text { None } & 100\end{array}$

EDTA $(1.5 \mathrm{mM})$

Cyanide $(2 \mathrm{mM}) \quad<2$

Acetylene (10\% by vol.) $\quad 4.3$

$\mathrm{Cu}^{2+}(400 \mu \mathrm{M})$

${ }^{a}$ The pMMO was solubilized with a detergent /protein weight ratio of 1.7, and pMMO activity was measured in the presence of $7.5 \mathrm{mM}$ duroquinol. 


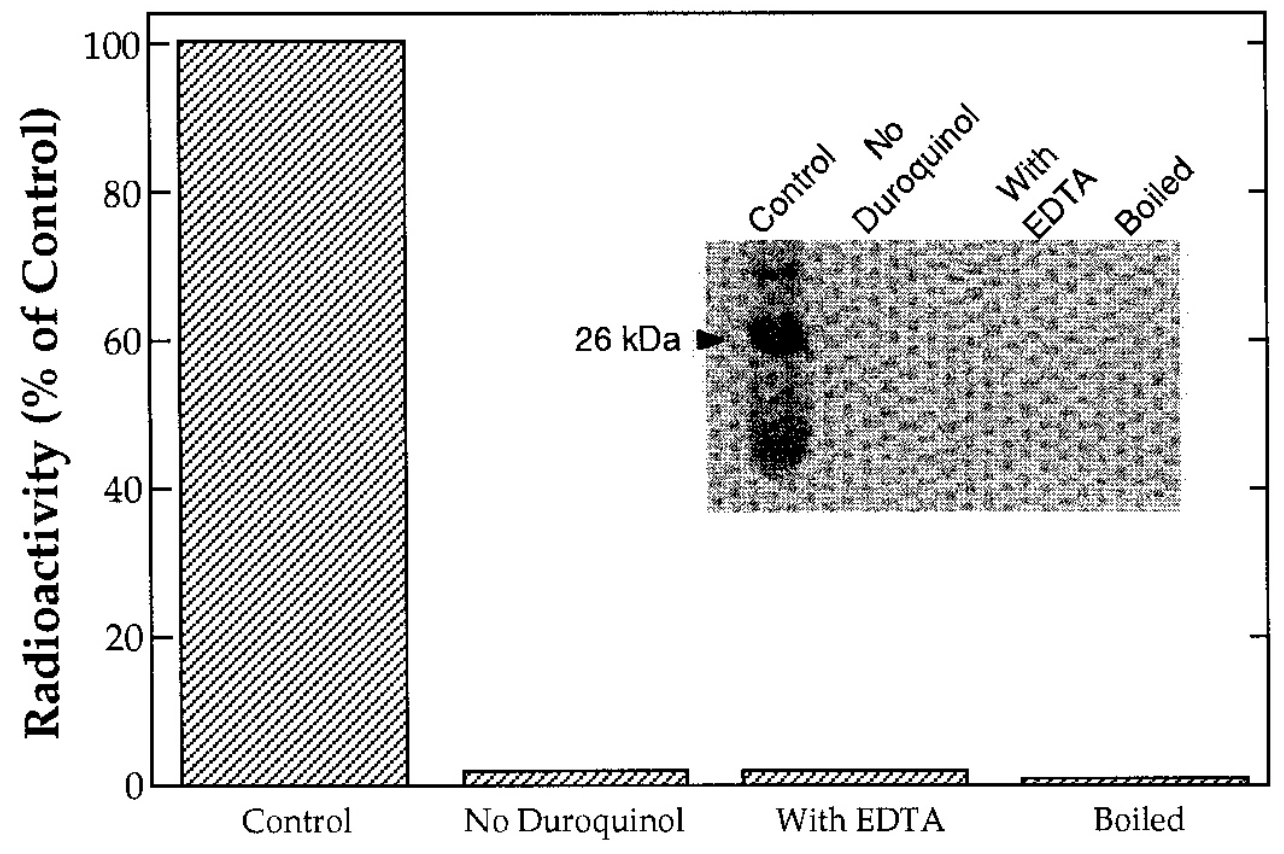

Figure 4

Radiolabeling of detergent solubilized pMMO by $\left[{ }^{14} \mathrm{C}\right]$ acetylene requires quinol and catalytically-competent pMMO. The bar graph shows the relative radioactivity of the soluble fraction after incubation with $\left[{ }^{14} \mathrm{C}\right]$ acetylene with and without duroquinol, with duroquinol plus $2 \mathrm{mM}$ EDTA, and with duroquinol after briefly boiling the sample. The radioactivity of the control fraction was $2.0 \times 10^{6} \mathrm{CPM}$. The inset is an auto-radiogram showing the $26 \mathrm{kDa}$ peptide that is predominantly labeled during incubation of the soluble fraction with duroquinol and $\left[{ }^{14} \mathrm{C}\right]$ acetylene. The high molecular weight band has a mass of $\sim 50 \mathrm{kDa}$, and the low molecular weight band ran with the dye front. 


\section{DISCUSSION}

\section{Reduction of pMMO by Quinols in vitro}

The membrane-bound form of MMO remains poorly characterized, primarily due to the lack of methods for purification of the enzyme. Purification procedures cannot be developed however, unless the enzyme can be solubilized from the membrane in an active form. In this communication we have shown that pMMO can be solubilized with lauryl maltoside, and it retained high activity if duroquinol or decyl-plastoquinol are present as the reductant (Table I, Figure 2). Approximately 25\% of the total quinoldependent activity of whole cells is retained in the detergent-soluble fraction, with a quinol-dependent specific activity comparable to that of whole cells and membranes (Table I), indicating that the catalytic properties of the enzyme are unaffected by solubilization. Moreover, detergent-solubilized and membrane-bound pMMO are completely analogous with respect to stimulation by copper; inhibition by EDTA, cyanide, and acetylene (Table II); and irreversible binding of $\left[\mathrm{C}^{14}\right]$ acetylene (Fig 4). The only significant change that occurs upon solubilization is that pMMO can no longer use $\mathrm{NADH}$ as electron donor, whereas the ability to utilize quinols is unaffected (Table I).

The relative ability of these quinols to support pMMO activity (Table I), provides insight into the mechanism and physiological relevance of this redox reaction. One mechanism for exogenous quinols to serve as electron donors to membrane-bound enzymes is by reducing the endogenous quinone pool. The rate of this type of mechanism depends primarily on thermodynamic driving force (20), hence it should be inversely related to the redox potential of the exogenous quinol. This mechanism was proposed for the reduction of AMO by quinols in whole cells of Nitrosomonas europea, 
since activity with methyl-substituted quinols was inversely related to their redox potentials (24). In the case of pMMO we observe no such correlation: duroquinol and decyl-plastoquinol have potentials similar to the ubiquinol and menaquinol analogs but are significantly more effective at reducing pMMO (Table I). Furthermore, the detergent-solubilized fraction should be depleted of endogenous quinols, yet pMMO activity with duroquinol and decyl-plastoquinol is not altered (Table I). Thus, it seems likely that these exogenous quinols reduce pMMO by binding directly to the catalytic core of the enzyme, or to a reductase that remains bound to pMMO after solubilization.

The ability of these exogenous quinols to directly reduce pMMO in vitro may result from their similarity to the natural reductant for pMMO in vivo (i.e., an endogenous quinol). Alternatively, reduction of pMMO by exogenous quinols in vitro may be adventitious, with no physiological relevance to the natural reductant. In this regard, we note that the most hydrophobic quinols are al so the most effective reductants for pMMO (Table I). It is conceivable that the greater hydrophobicity of duroquinol and decylplastoquinol enhances their access to the active site cofactors. However, the difference in hydrophobicity of these quinols is small, whereas the difference in pMMO activity is great (Table I). Furthermore, the selectivity of pMMO for specific quinols is not consistent with this type of non-specific reduction. For example, duroquinol is much more effective than menaquinol despite similar size, redox potential, and hydrophobicity (Table I). In addition, the active site of pMMO is not readily accessible: hydrocarbons larger than butane are not oxidizable by pMMO and aromatic reductants, such as methylviologen, benzyl-viologen, and TMPD are unable to reduce pMMO. The possibility that the exogenous quinols non-specifically reduce a redox cofactor remote from the pMMO 
active site also seems inconsistent with the much greater activity of duroquinol and decyl-plastoquinol, relative to the other quinols. Although the dimethyl- and trimethylquinols are structurally similar duroquinol and decyl-plastoquinol, they are not effective reductants for pMMO (Table I), perhaps a result of the relatively high redox potentials of the former two quinols. Thus, it appears the greater activity of duroquinol and decylplastoquinol results from their similarity in structure and redox potential to quinols that reduce $\mathrm{pMMO}$ in vivo.

In whole cells, membranes, and detergent-solubilized fractions the pMMO activity is much greater with duroquinol and decyl-plastoquinol as electron donor, relative to decyl-ubiquinol (Table I). This is also the case for enzymes that are specifically reduced by plastoquinols (13), whereas the opposite is true for enzymes that are specific for ubiquinols (14). Therefore, if the natural reductant for $\mathrm{pMMO}$ is a quinol, it is probably plastoquinol, rather than ubiquinol. Several investigations of the quinone content of methanotrophs $(30,6)$, have demonstrated that $M$. capsulatus and other methanotrophs contain significant amounts of ubiquinone, and no menaquinone. Unfortunately, no mention is made of plastoquinone in these reports. Thus, which plastoquinones are present in $M$. capsulatus, if any, remains a subject for future study.

\section{Reduction of pMMO by Quinols in vivo}

As we show in Table I and Fig. 2, NADH can provide reducing equivalents for pMMO in whole cells and membranes, but not after detergent solubilization. In contrast, duroquinol and decyl-plastoquinol are at least as effective before solubilization as after (Table I). This, together with the specificity for these particular quinols, suggests that the quinone pool is required for reduction of pMMO by NADH. Thus, we propose the 
existence of an electron-transport chain for the reduction of pMMO in vivo, as illustrated in Scheme 1.

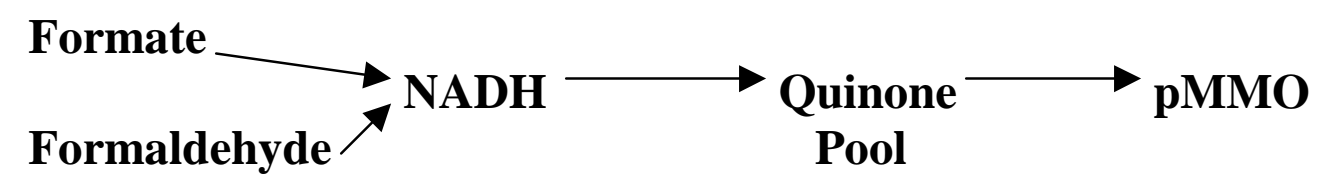

Scheme 1: Proposed electron-transport chain for reduction of pMMO in M. capsulatus.

Methanotrophs are known to generate NADH from the oxidation of formaldehyde and formate (3). In this putative electron-transport chain, NADH is utilized to reduce the quinone pool, which subsequently reduces pMMO. The NADH dehydrogenase inhibitor amytal is reported to inhibit NADH-dependent pMMO activity in membranes from Methylosinus trichosporium (22), consistent with this type of electron-transport chain. The pMMO in membranes from these latter bacteria can obtain reducing equivalents from succinate (7), suggesting that a succinate dehydrogenase similar to complex II of mitochondria catalyzes reduction of the quinone pool by succinate. M. capsulatus apparently lacks this succinate dehydrogenase, since succinate supports neither pMMO activity or oxygen uptake in membranes from these bacteria (data not shown). Thus, transfer of reducing equivalents from low potential electron donors (NADH, formate, succinate, etc.) to pMMO appears to be mediated by quinones in both of these methanotrophs. As discussed above, our results are consistent with direct reduction of pMMO by exogenous quinols, as a result of their similarity to a natural plastoquinol reductant. However, we cannot rule out the requirement for other redox proteins to catalyze reduction of pMMO by quinols in vivo. If such a quinol/pMMO reductase exists 
it appears to be tightly bound to pMMO, remaining associated after detergent solubilization.

The requirement for quinols to mediate reduction of pMMO by NADH could explain why previous attempts to solubilize the enzyme were unsuccessful. Detergent solubilization apparently inactivates the enzyme that catalyzes reduction of quinones by $\mathrm{NADH}$, or separates this enzyme from pMMO, so reduction by NADH is no longer possible. The results of Smith and Dalton indicate that the electron transfer chain from $\mathrm{NADH}$ to pMMO can be reassembled in phospholipid vesicles, but it is not stable enough to survive fractionation (25). The use of quinols to assay pMMO activity during purification should eliminate the need to co-purify the additional enzymes needed for reduction by NADH, and facilitate purification of the catalytic core of pMMO.

\section{ACKNOWLEDGEMENTS}

We would like to thank Dr. Diana Beattie and members of her laboratory for their assistance with preparing reduced quinones. We would also like to thank Subir Mitra and Elias Halvas for their technical assistance with many of the experiments described in this report. 


\section{REFERENCES}

1. Akent'eva, N.F., and R.I. Gvozdev. 1988. Purification and physiochemical properties of methane monooxygenase from membrane structures of Methylococcus capsulatus. Microbiology. 53:91-96.

2. Anthony, C. 1982. The Biochemistry of Methylotrophs, Academic Press, London.

3. Anthony, C. 1986. Bacterial oxidation of methane and methanol. Adv. Microb. Physiol. 27:113-209.

4. Arciero, D.M., T. Vanneli, M. Logan, and A.B. Hooper. 1989. Degradation of trichloroethylene by the ammonia-oxidizing bacterium Nitrosomonas europaea. Biochem. Biophys. Res. Comm. 159:640-643.

5. Burrows, K.J., A. Cornish, I. J. Scott, and I.J. Higgins. 1984 Substrate specificities of the soluble and particulate methane mono-oxygenases of Methylosinus trichosporium OB3b. J. Gen. Microbiol. 130: 3327-3333.

6. Collins, M.D., O.W. Howarth, and P.N. Green. 1986. Isolation and structural determination of a novel coenzyme from a methane oxidizing bacterium. Arch. Microbiol. 146:263-266.

7. Cornish, A., J. MacDonald, K.J. Burrows, T.S. King, D. Scott, and I.J. Higgins. 1985. Succinate as an in vitro electron donor for the particulate methane monooxygenase of Methylosinus trichosporium OB3b. Biotechnol. Lett. 7:319-324.

8. Dawson, R.M.C., D.C. Elloitt, W.H. Elliott, and K.M. Jones. 1986. Data for Biochemical Research, $3^{\text {rd }}$ Edition, Clarendon Press, Oxford. 
9. DiSpirito, A.A., J. Gulledge, A.K. Shiemke, J.C. Murrell, M.E. Lidstrom, and C.L. Krema. 1992. Trichloroethylene oxidation by the membrane associated methane monooxygenase in type I, type II and type X methanotrophs. Biodegradation 2:151-164.

10. Ensign, S.A., M.R. Hyman, and D.F. Arp. 1993. In vitro activation of ammonia monooxygenase from Nitrosomonas europaea by copper. J. Bacteriol. 175:1971-1980.

11. Hanson, R.S., A.I. Netrusov, and K. Tsuji. 1991. Pp. 1-27. In A.H. Balow, G. Truper, M. Dwarkin, W. Hander, and K.H. Schleifer, (eds.), The Prokaryotes, $2^{\text {nd }}$ ed., Springer-Verlag, New York.

12. Higgins, I.J., D. Scott, and R.C. Hammond. 1980. New findings in methaneutilizing bacteria highlight their importance in the biosphere and their commercial potential. Nature 286:561-564.

13. Izawa, S. 1980. Acceptors and donors for chloroplast electron transport. Meth. Enzymol. 69:413-434.

14. Lenaz, G., A. DeSantis, and E. Bertoli. 1985. Pp.165-201. In G. Lenaz (ed) Coenzyme Q, John Wiley \& Sons, Chichester, UK.

15. Mason, H.S. 1965. Oxidases. Ann. Rev. Biochem. 34: 595-634.

16. Nguyen, H.T., A.K. Shiemke, S.J. Jacobs, B.J. Hales, M.E. Lidstrom, and S.I. Chan. 1994. The nature of the copper ions in the membranes containing the particulate methane monooxygenase from Methylococcus capsulatus (Bath). J. Biol. Chem. 269:14995-15005. 
17. Periana, R.A., D.J. Taube, E.R. Evitt, D.G. Loffler, P.R. Wentrcek, G. Voss, and T. Masuda. 1993. A mercury-catalyzed, high-yield system for the oxidation of methane to methanol. Science 259:340-343.

18. Prior, S.D., and H. Dalton. 1985. The effect of copper ions on membrane content and methane monooxygenase activity in methanol-grown cells of Methylococcus capsulatus (Bath). J. Gen, Microbiol. 131:155-164.

19. Prior, S.D., and H. Dalton. Acetylene as a suicide substrate and active site probe for methane monooxygenase from Methylococcus capsulatus (Bath). FEMS Microbiol. Lett. 29:105-109.

20. Rich, P.R. 1981 Electron transfer reactions between quinols and quinones in aqueous and aprotic media. Bioch. Biophys. Acta 637:28-33.

21. Rosenzweig, A.C., C.A. Frederick, S.J. Lippard, and P. Nordlund. 1993. Crystal structure of a bacterial non-haem iron hydroxylase that catalyses the biological oxidation of methane Nature 366: 537-543.

22. Scott, D., J. Brannan, and I.J. Higgins. 1981. The effect of growth conditions on intracytoplasmic membranes and methane mono-oxygenase activities in Methylosinus trichosporium OB3b. J. Gen. Microbiol. 125:63-72.

23. Semprini, L., P.V. Roberts, G.D. Hopkins, and P.L. McCarty. 1990. A field evaluation of in-situ biodegradation of chlorinated ethenes: part 2, results of biostimulation and biotransformation experiments. Groundwater 28:715-727.

24. Shears, J.H., and P.M. Wood. 1986. Tri- and tetra methylhydroquinone as electron donors for ammonia monooxygenase in whole cells of Nitrosomonas europae. FEMS Microbiol. Lett. 33:281-284. 
25. Smith, D.D.S., and H. Dalton 1989. Solubilisation of methane-monooxygenase from Methylococcus capsulatus (Bath). Eur. J. Biochem. 182:667-671.

26. Stainthorpe, A.C., J.C. Murrell, G.P.C. Salmond, H. Dalton, and V. Lees. 1989. Molecular analysis of methane monooxygenase from Methylococcus capsulatus (Bath). Arch. Microbiol. 152:154-159.

27. Stainthorpe, A.C., V. Lees, G.P.C. Salmond, H. Dalton, and J.C. Murrell. 1990. The methane monooxygenase gene cluster of Methylococcus capsulatus (Bath). Gene 91:27-34.

28. Stanley, S. H., S.D. Prior, D.J. Leak, and H. Dalton. 1983. Copper stress underlies the fundamental change in intracellular location of methane monooxygenase in methane oxidizing organisms. Biotechnol. Lett. 5:487-492.

29. Tonge, G.M., D.E.F. Harrison, C.J. Knowles, and I.J. Higgins. 1975. Properties and partial purification of the methane-oxidising enzyme system from Methylosinus trichsporium. FEBS Lett. 58:293-299.

30. Urakami, T. and K. Komagata. 1986. Occurrence of isoprenoid compounds in gram negative methanol, methane, and methylamine utilizing bacteria. J. Gen. Microbiol. 32:317-341.

31. Wackett, L.P., and D.T. Gibson. 1988. Degradation of trichloroethylene by toluene dioxygenase in whole-cell studies with Pseudomonas putida F1. Appl. Environ. Microbiol. 54:1703-1708.

32. Wackett, L.P., G.A. Brusseau, S.R. Householder, and R.S. Hanson. 1989. Survey of microbial oxygenases: trichloroethylene degradation by propaneoxidizing bacteria. Appl. Environ. Microbiol. 55:2960-2964. 


\section{CHAPTER FOUR}

Isolation, Purification, and Characterization of Endogenous Quinones and Their Use in Support of pMMO Activity in vitro 


\section{INTRODUCTION}

The particulate form of methane monooxygenase (pMMO) initiates the utilization

of methane in methanotrophs (3). It performs this reaction by accepting reducing equivalents from an, as yet, unknown source. It had previously been believed that the two reducing equivalents came directly from NADH. However, solubilization of the enzyme (with lauryl maltoside) resulted in complete loss of activity when using NADH as the reductant. Apparently, either an intermediary component of the chain between $\mathrm{NADH}$ and pMMO was lost during solubilization or the enzyme itself underwent a detergent-driven, irreversible conformational change.

Our initial model of the respiratory chain of Methylococcus capsulatus depicts several proteins of interest, transferring reducing equivalents from the oxidation of methane ultimately to molecular oxygen, reducing it to water. However, experimental characterization of the chain has largely been limited to the interaction of pMMO with exogenous quinols (previous chapter) (18). Recently, Cook and Shiemke purified a NADH-dependent type II dehydrogenase capable of reducing exogenous quinols in vitro. Prior to this, individual studies of methanol dehydrogenase (9) and a $\mathrm{caa}_{3}$-type oxidase (7) summarized what was known about the respiratory enzymes in $M$. capsulatus. The study of endogenous respiratory lipids (i.e., quinones) has been ignored, to date, with the exception of a quinone purification from $M$. capsulatus by Collins, et al. (4). Subsequently, Nguyen, et al. reported the isolation of a crude quinone extract from $M$. capsulatus, but no details of the purification or characterization of their extract were reported (15). 
Several types of quinones are known to be important intermediates in the transfer of reducing equivalents through the respiratory chains of living systems $(11,16)$. Typically, the ubiquinones are found within prokaryotic and eukaryotic cells, while plastoquinones and menaquinones are spread largely among plants and bacteria. Rhodoquinone, on the other hand, has so far only been found in a subset of photosynthetic bacteria. (14). Ubiquinone-10 $\left(\mathrm{CoQ}_{10}\right)$ is the mobile electron carrier found in eukaryotic horse heart mitochondria (20) and $\mathrm{CoQ}_{9}$ is found in the mitochondria of rats (13). Plastoquinone is found in spinach chloroplasts (12) and menaquinone is the agent located in a wide variety of bacteria (13).

This chapter reports the isolation, purification, and characterization of three endogenous quinones, two of which have not previously been reported. The crude extract as well as the purified form of one of the quinones were assessed for their ability to support pMMO activity in vitro. This is the first report to show endogenous quinonedependence of pMMO activity. 


\section{RESULTS}

\section{Isolation, Purification, and Characterization of the Endogenous Quinones from the Membrane Fraction of pMMO-expressing Methylococcus capsulatus}

As seen in Figure 1, reversed phase chromotography of the $n$-pentane extract yielded several prominent, well-resolved peaks. The large peak at approximately 3.8 minutes is due to a preservative (BHT) added to prevent oxidation of the aliphatic isoprenoid tail. The remaining six prominent peaks as well as a pooled sample of the regions between the peaks were analyzed by UV-visible spectroscopy as well as GC/MS to ascertain the presence and/or level of quinone compounds exhibited.

Analysis of peak \#1 (9.1 minutes), peak \#2 (12.8 minutes), and peak \#3 (14.3 minutes) by UV-visible or GC/MS showed no evidence of the presence of quinones (data not shown). The instrumental GC/MS library search resulted in an array of possibilities for these first three peaks, some of which were lipids, but none of which were quinones. Similarly, the pooled regions between the prominent peaks also suggested no quinones were present at these points.

Conversely, the peaks at 19.0 minutes, 26.0 minutes, and 28.0 minutes (from this point forward referred to as peak \#4, peak \#5, and peak \#6, respectively) exhibited entirely different results. Peak \#4, the major component of the extract, was the focal point of this work. An aliquot of this peak was chromatographed via silica TLC yielding a single spot which co-chromatographed with standard coenzyme $\mathrm{Q}_{10}$. However, no indication that menaquinone (vitamin $\mathrm{K}_{2}$ ) was present when comparing to such standard.

The purified fraction of peak \#4 was then mixed with an array of ubiquinone standards $\left(\mathrm{CoQ}_{6}, \mathrm{CoQ}_{7}, \mathrm{CoQ}_{9}\right.$, and $\left.\mathrm{CoQ}_{10}\right)$ in methanol, and the resultant chromatogram 


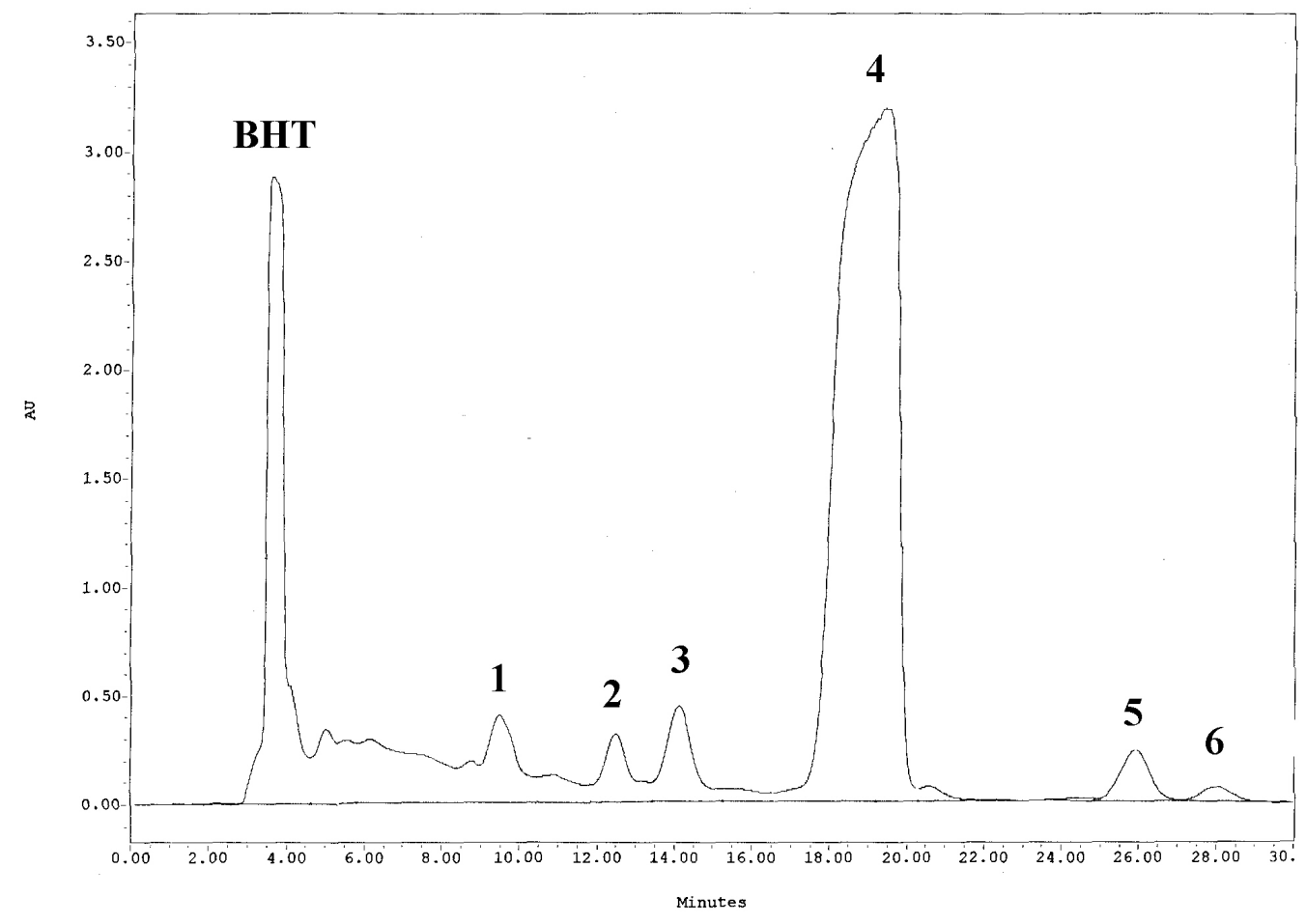

Figure 1. Reversed-phase chromatograph of crude $n$-pentane quinone extract from Methylococcus capsulatus (Bath). Peaks 1-3 are non-quinone organics, peak \#4 is 18'methylene $\mathrm{CoQ}_{8}$, peak \#5 is $\mathrm{CoQ}_{9}$, and peak \#6 is 18 '-methylene $\mathrm{CoQ}_{9}$. 
is depicted in Figure 2 (A). Peak \#4 eluted between $\mathrm{CoQ}_{7}$ and $\mathrm{CoQ}_{9}$. The $\log _{10}$ of the retention time for each standard was then plotted vs. number of isoprene units in each respective tail (Figure 3). Interpolation of the graph of retention time vs. isoprene units yielded an experimental value of 8.1 isoprenes in the tail of peak \#4. Additionally, the UV-visible spectrum of peak \#4 exhibited the same maxima and general envelope shape as those from the standards (Figure 2, B).

Following HPLC purification, peaks \#4, 5, and 6 were analyzed via gas chromotography/ mass spectrometry (Figures 4-6). All three analytes exhibit fragment ions at $\mathrm{m} / \mathrm{e}=235$ (base peak) and $\mathrm{m} / \mathrm{e}=197$ indicative of ions from the ubiquinone class of compounds as illustrated in Figure 7 (4). There is also a prominent $M+2$ ion in each of the spectra indicative of the dismutations resulting in the quinol form. The rhythmic pattern between $\mathrm{M}-15$ and $\mathrm{m} / \mathrm{e}=235$ were not apparent for the loss of successive isoprene units. None of the spectra recorded showed evidence of plastoquinone, which would exhibit prominent signals at $\mathrm{m} / \mathrm{e}=151$ and $\mathrm{m} / \mathrm{e}=189$, suggesting that Methylococcus capsulatus does not produce this class of quinones.

The parent ion in peak \# 4 was identical to that reported previously by Collins \& Green in 1985 , who concluded that it was $18^{\prime}$-methylene $\mathrm{CoQ}_{8}$. However, the parent ions observed in peaks \#5 and \#6 differed from anything previously reported for Methylococcus capsulatus. The parent ion observed for peak \# 5 was consistent with that of ubiquinone-9 $\left(\mathrm{CoQ}_{9}\right)$ (as was the rest of the mass spectrum) within experimental error. The published parent ion is reported at $\mathrm{m} / \mathrm{e}=794$ while that observed in this data occurred at $\mathrm{m} / \mathrm{e}=795$. Peak \# 6 resulted in a parent ion of 808 , approximately 14 mass 

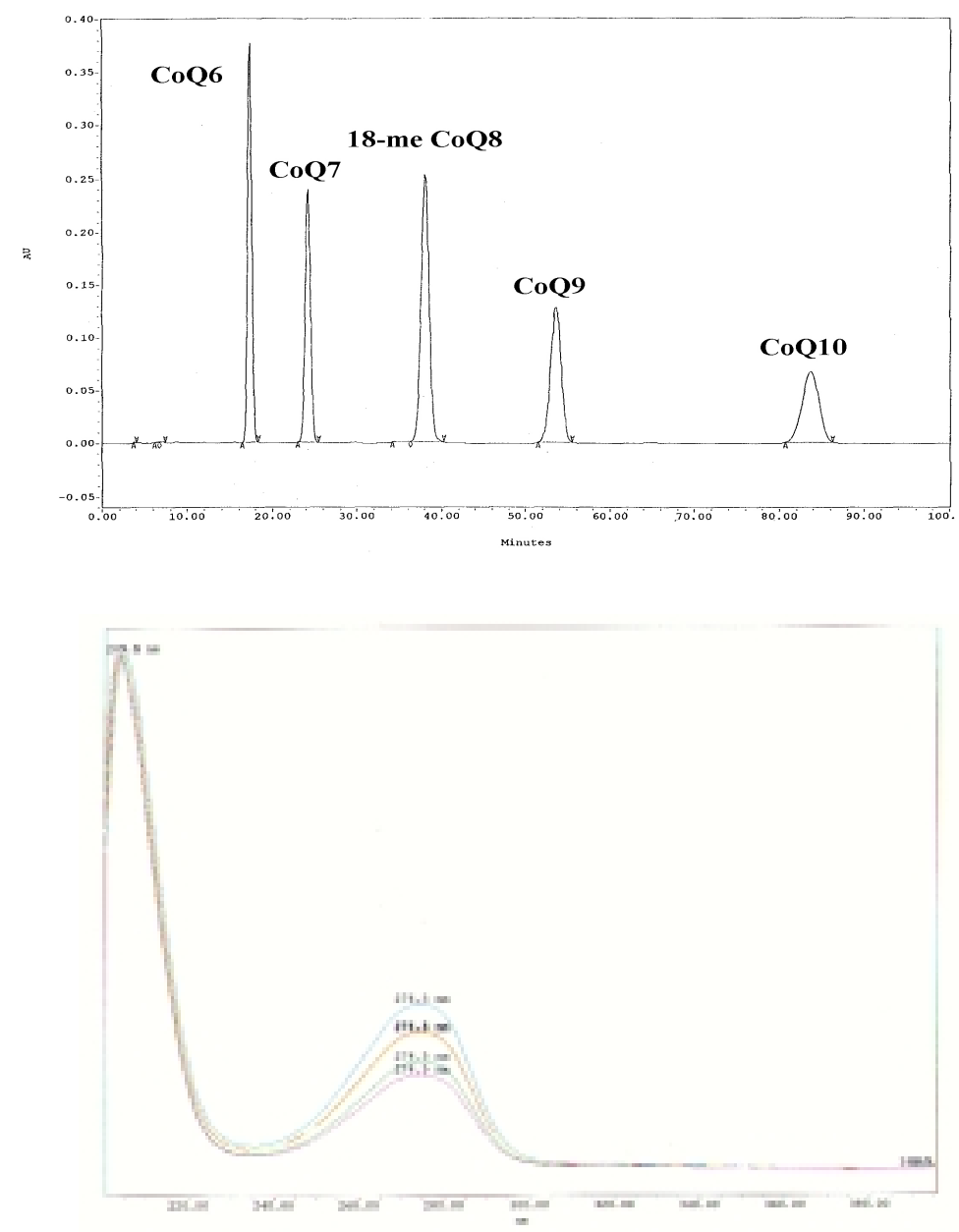

Figure 2. A) Reversed-phase chromatograph of purified peak \#4 co-chromatographed with quinone standards. The flow rate was reduced two-fold from that used in Figure 1. B) UV spectra of the standards and peak\#4 (18'-methylene $\left.\mathrm{CoQ}_{8}\right)$ were recorded at the apex of the respective peaks (bottom) to note similarity. $\mathrm{CoQ}_{6}(\mathrm{Blue}), \mathrm{CoQ}_{7}(\mathrm{Red}), 18$ 'methylene $\mathrm{CoQ}_{8}$ (Yellow), $\mathrm{CoQ}_{9}$ (Green), $\mathrm{CoQ}_{10}$ (Purple). 


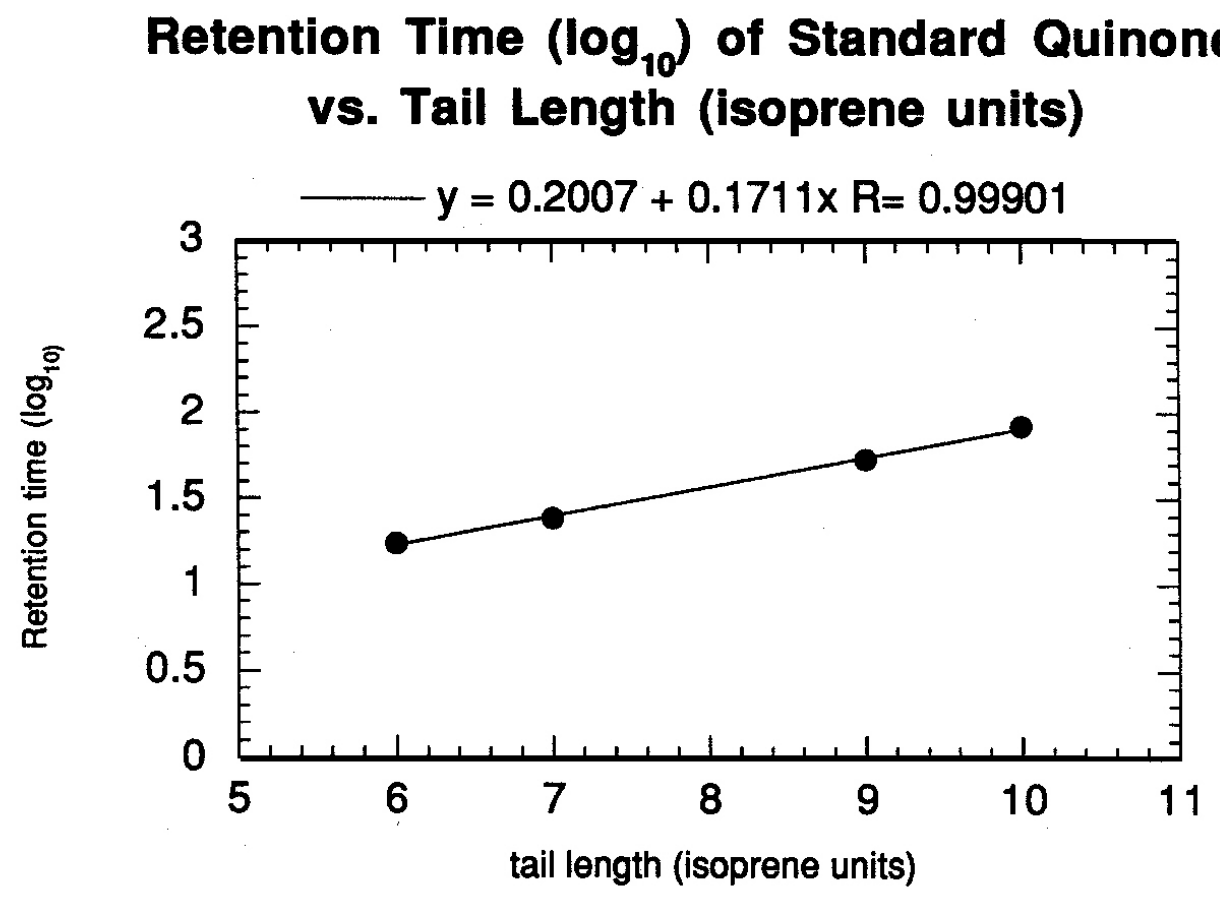

Figure 3. $\log _{10}$ Retention Time vs. Tail Length (\# Isoprene Units). Data values for the four quinone standards are shown with a regression line calculated. Extrapolation of the retention time for peak \# 4 yields a result of 8.1 isoprenes in the tail of peak \#4. 


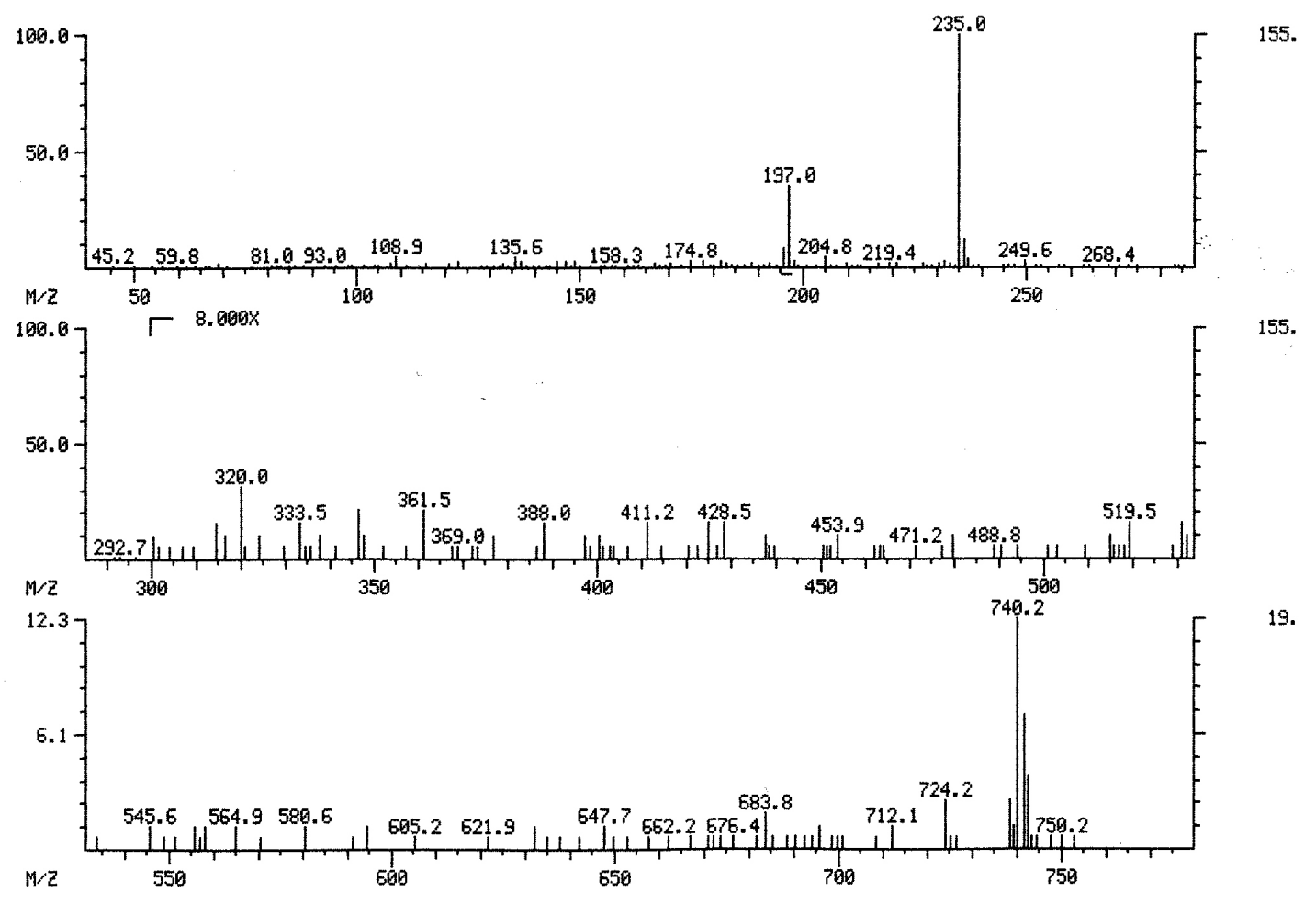

Figure 4. GC mass spectrum of 18'-methylene $\mathrm{CoQ}_{8}$. Molecular ion occurs at $\mathrm{m} / \mathrm{e}=$ 740.2, and base peak at $\mathrm{m} / \mathrm{e}=235.0$. Also, note the molecular ion at $\mathrm{m} / \mathrm{e}=197.0$. 


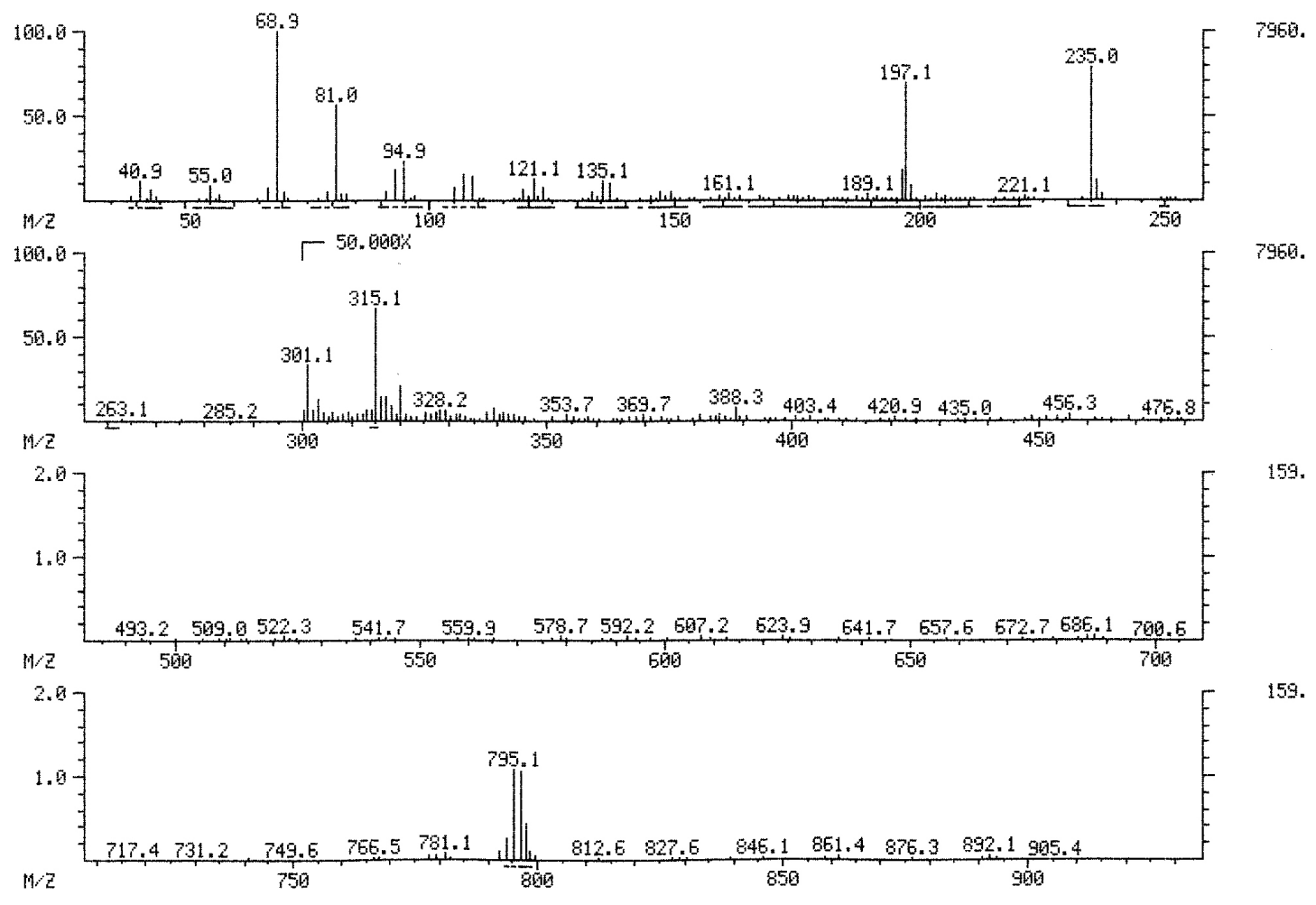

Figure 5. GC mass spectrum of $\mathrm{CoQ}_{9}$. Molecular ion occurs at $\mathrm{m} / \mathrm{e}=795.1$ and base peak at 235.0. Also, note the molecular ion at $\mathrm{m} / \mathrm{e}=197.1$. 


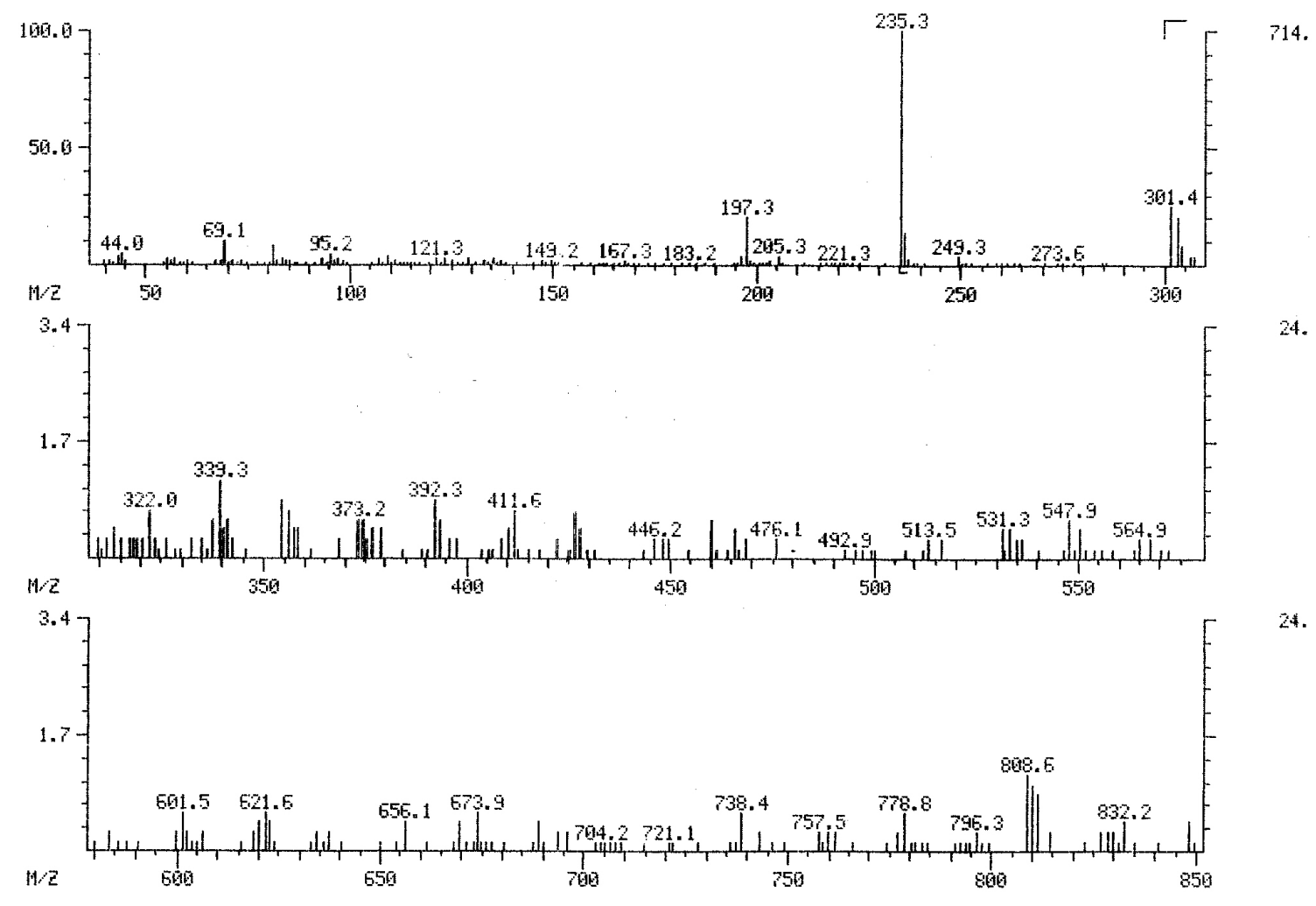

Figure 6. GC mass spectrum of 18 '-methylene $\mathrm{CoQ}_{9}$. Molecular ion occurs at $\mathrm{m} / \mathrm{e}=$ 808.6, and base peak at $\mathrm{m} / \mathrm{e}=235.3$. Also, note the molecular ion at 197.3. 


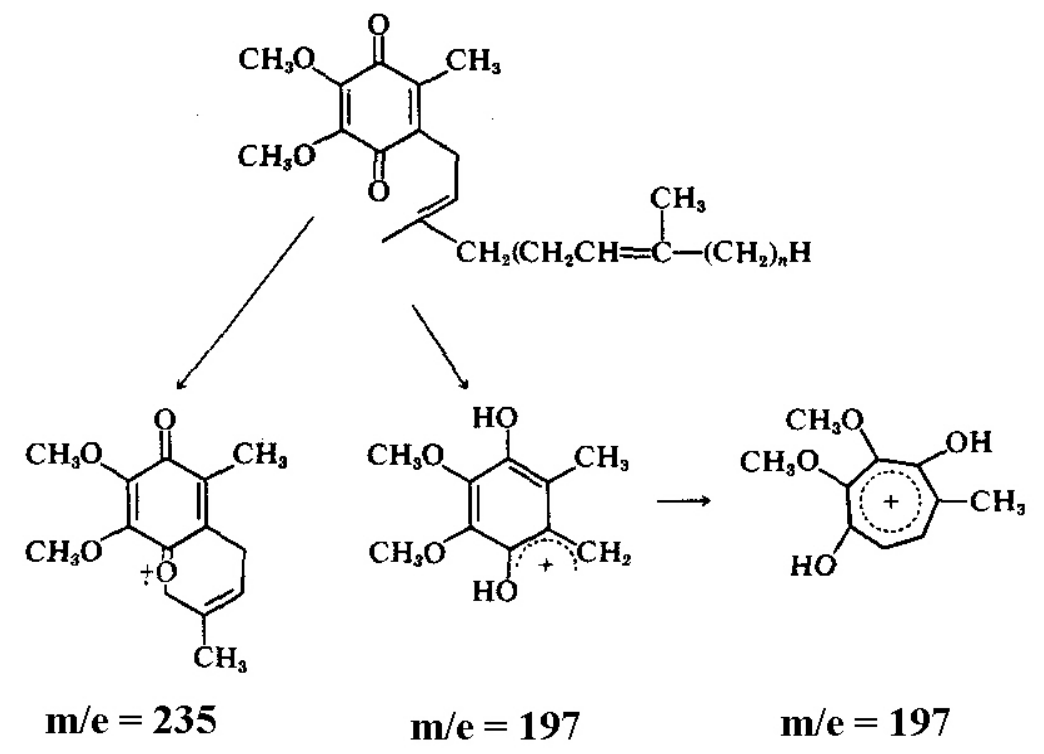

Figure 7. Fragmentation scheme for the ubiquinone class of compounds. General molecular structure is given at the top as well as the structures of the base peak $(\mathrm{m} / \mathrm{e}=$ 235) and other prominent ions occurring at $\mathrm{m} / \mathrm{e}=197.0$. 
units higher than that for peak \# 5. It is likely that this analyte would be $18^{\prime}$-methylene $\mathrm{CoQ}_{9}$.

The ${ }^{1} \mathrm{H}$ and ${ }^{13} \mathrm{C}$ NMR spectra as well as COSY spectrum of peak \#4 are illustrated in Figures 8, 9 and 10. These spectra confirm the identity of this sample as 18'methylene $\mathrm{CoQ}_{8}$. Of particular note were signals in the ${ }^{13} \mathrm{C}$ spectrum. Signals at $154.555 \mathrm{ppm}$ and $107.468 \mathrm{ppm}$ were indicative of the vinylic $\left(\mathrm{C}=\mathrm{CH}_{2}\right)$ group. Other signals at 25.7278ppm, 35.6146ppm, 107.468ppm, 20.1465ppm, 38.4198ppm, and $32.4187 \mathrm{ppm}$ confirmed the position of the vinyl group on the $5^{\text {th }}$ isoprene from the ring. Other confirmatory structure information was gained from the ${ }^{1} \mathrm{H}$ NMR (such as the presence of the methoxy protons) and COSY spectra (presence and/or positions of aliphatic protons on the isoprene chain). The proposed structure for the endogenous 18'methylene $\mathrm{CoQ}_{8}$ is reproduced in Figure 11 with the carbon-13 shift assignments (ppm) noted for reference.

\section{Enzymatic Activity Assays}

Once the structure of the major quinone component was known, we next investigated what effect, if any, the endogenous quinone has on pMMO activity. Three different environments were analyzed with regards to pMMO activity: (I) whole cells, (ii) membranes, and (iii) solubilized membrane fraction.

As Table 1 illustrates, the highest specific activity was observed in whole cells, with formate as reductant. After lysing the cells, specific activity for pMMO drops significantly in the membrane fraction with $\mathrm{NADH}$ as reductant, as previously reported (Chapter 3). The specific activity drops still further (to $\sim 0$ ) after solubilization, when NADH is the reductant. We hypothesized that the quinone pool of M. capsulatus may be 


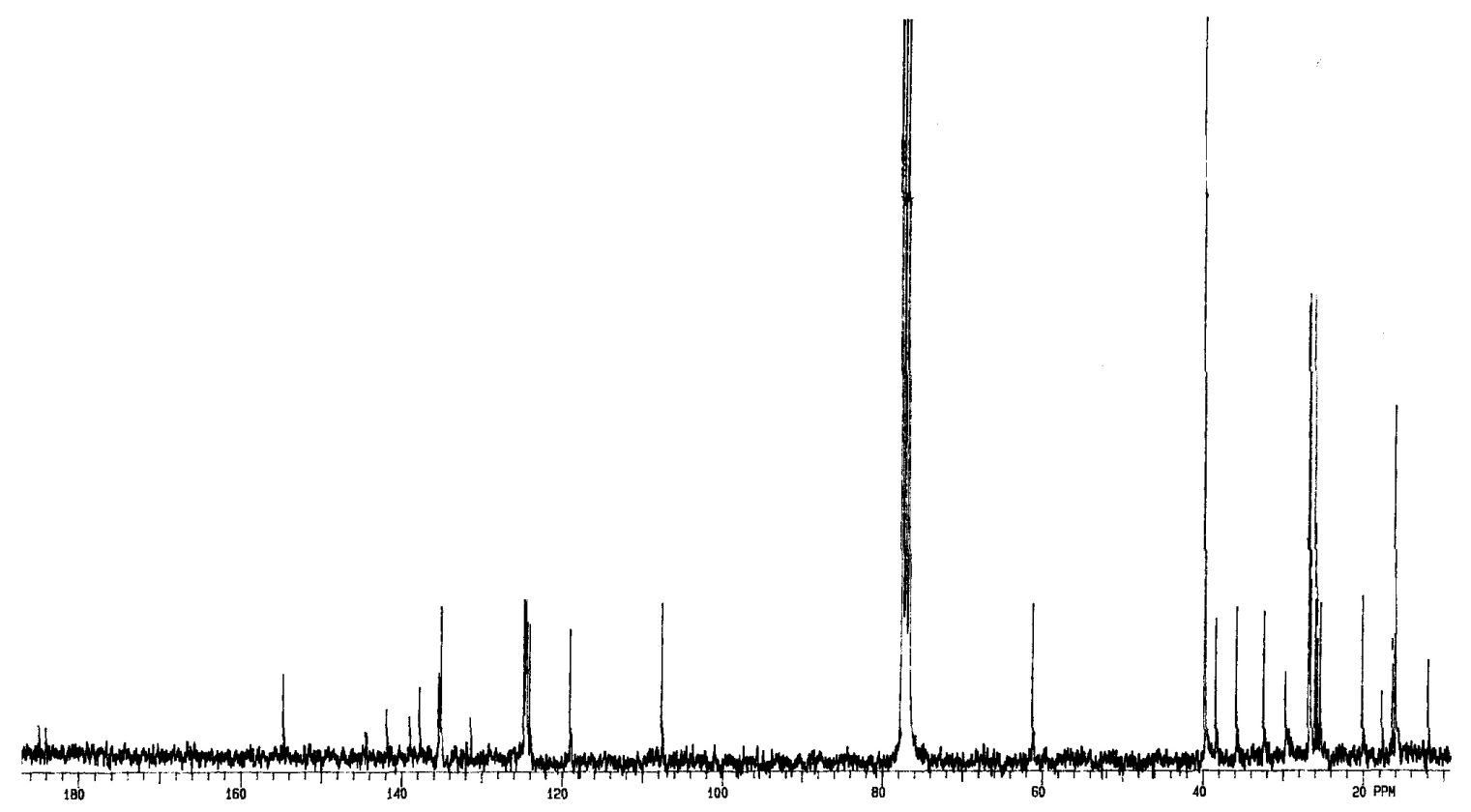

Figure 8. ${ }^{13} \mathrm{C}$ NMR spectrum of 18 '-methylene $\mathrm{CoQ}_{8}$ (Peak \#4). Solvent was $\mathrm{CDCl}_{3}$ and chemical shifts are expressed as ppm 


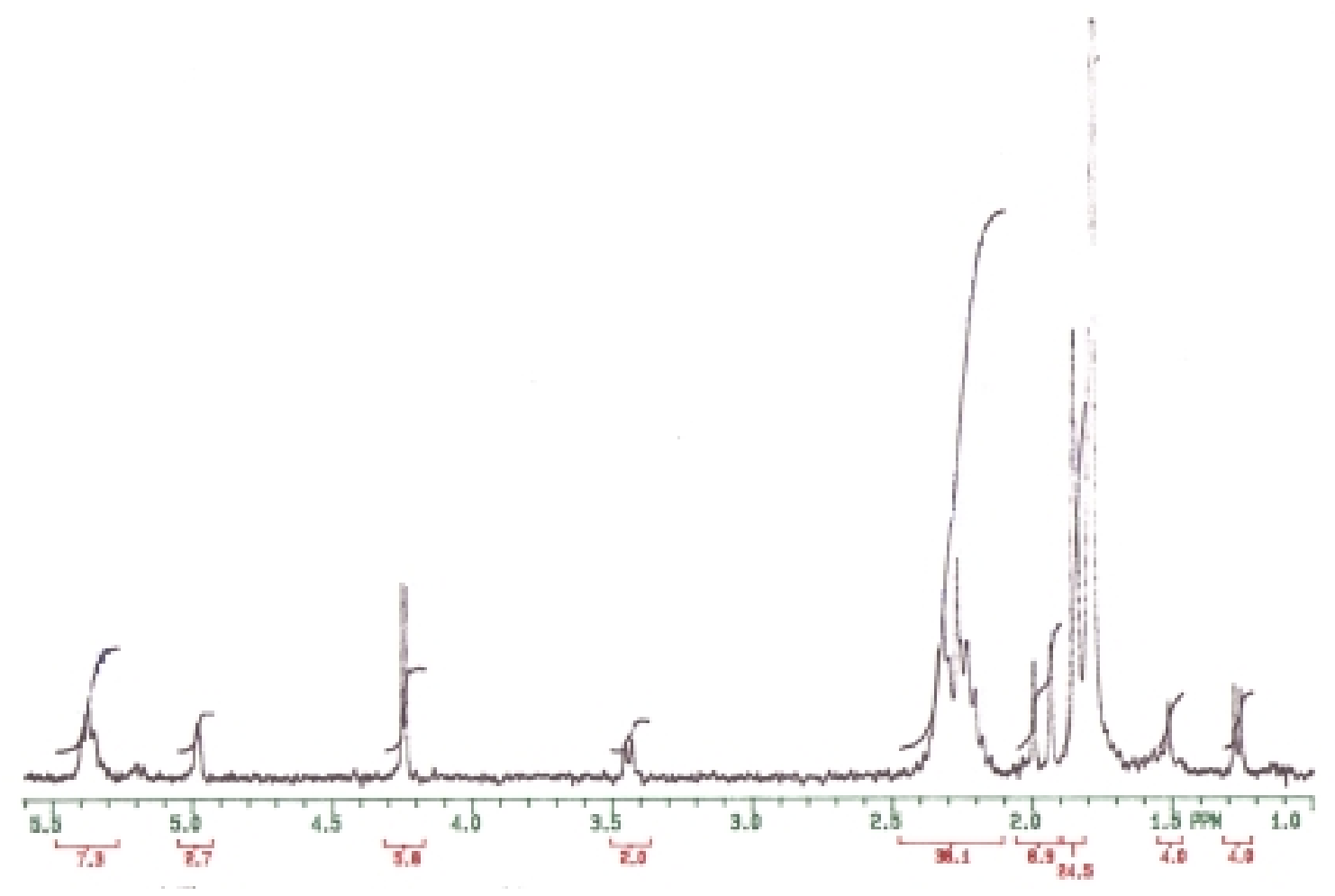

Figure 9. ${ }^{1} \mathrm{H}$ NMR spectrum of 18 '-methylene $\mathrm{CoQ}_{8}$ (Peak \#4). Solvent was $\mathrm{CDCl}_{3}$ and chemical shifts are expressed in ppm. Integration results are depicted below the spectrum in red. 


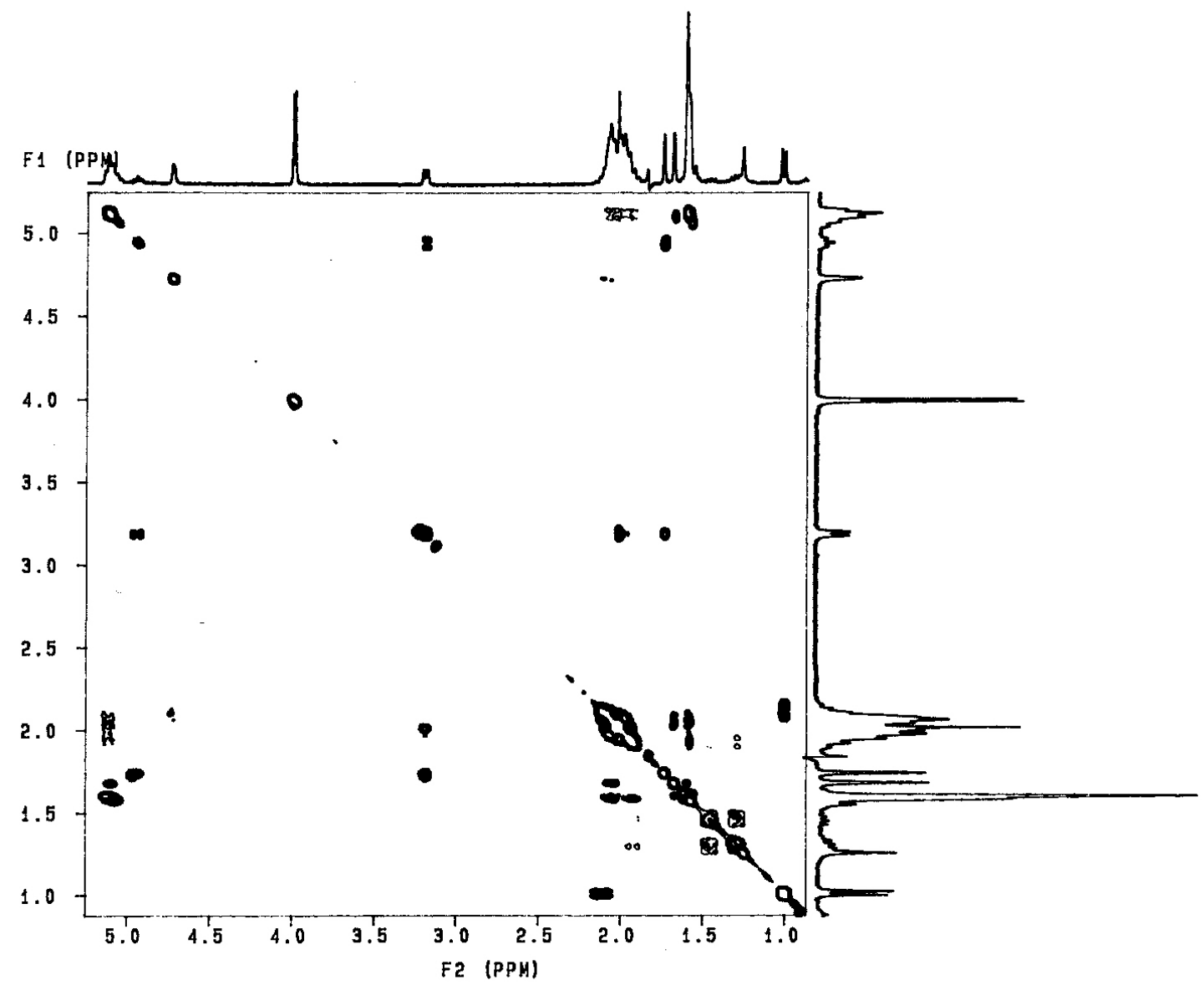

Figure 10. ${ }^{1} \mathrm{H}$ COSY spectrum of 18 '-methylene $\mathrm{CoQ}_{8}(\mathrm{Peak} \# 4)$. Solvent was $\mathrm{CDCl}_{3}$ and chemical shifts are expressed in ppm. Total acquisition time was 7 hours, 6.9 minutes. 


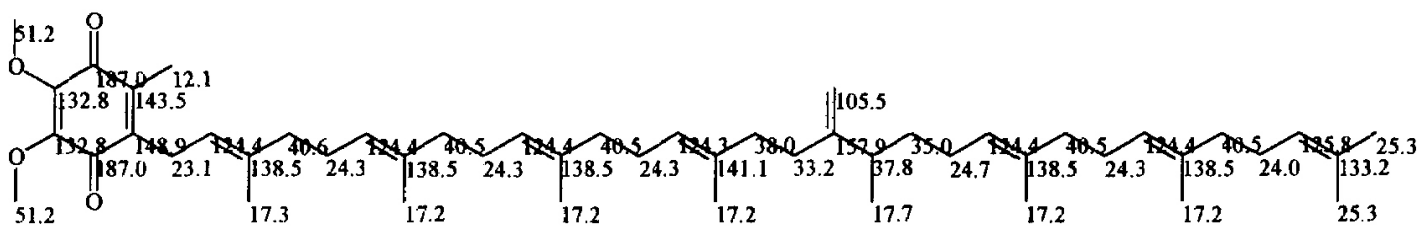

Figure 11. Proposed chemical structure of Peak \#4 (18'-methylene $\left.\mathrm{CoQ}_{8}\right)$ with theoretical chemical shifts. 
Table 1. Stimulatory Effects of Quinones on pMMO

\begin{tabular}{|c|c|c|}
\hline Sample & Reductant & Specific Activity $^{1}$ \\
\hline Whole Cells & Formate $(5 \mathrm{mM})$ & $38.3 \pm 2.4$ \\
\hline Whole Cells & Decyl-plastoquinol (5mM) & $6.1 \pm 0.4$ \\
\hline Whole Cells & Duroquinol $(5 \mathrm{mM})$ & $4.1 \pm 0.3$ \\
\hline Whole Cells & 18'-MQ8H2 (250uM) & $0.4 \pm 0.1$ \\
\hline Whole Cells & 18'-MQ8H2 (500uM) & $0.3 \pm 0.07$ \\
\hline Whole Cells & 18'-MQ8H2 (1mM) & $\sim 0$ \\
\hline Membranes & NADH $(5 \mathrm{mM})$ & $6.1 \pm 0.6$ \\
\hline Membranes & Decyl-plastoquinol $(5 \mathrm{mM})$ & $0.6 \pm 0.1$ \\
\hline Membranes & Duroquinol $(5 \mathrm{mM})$ & $2.1 \pm 0.2$ \\
\hline Membranes & 18'-MQ8H2 (250uM) & $\sim 0$ \\
\hline Membranes & 18'-MQ8H2 (500uM) & $0.2 \pm 0.05$ \\
\hline Membranes & 18'-MQ8H2 (1mM) & $0.4 \pm 0.03$ \\
\hline Soluble Fraction & NADH (10mM) & $\sim 0$ \\
\hline Soluble Fraction & Decyl-plastoquinol (10mM) & $4.9 \pm 0.3$ \\
\hline Soluble Fraction & Duroquinol $(10 \mathrm{mM})$ & $1.1 \pm 0.05$ \\
\hline Soluble Fraction & 18'-MQ8 (250uM) ${ }^{\mathrm{a}}$ & $0.8 \pm 0.04$ \\
\hline Soluble Fraction & 18'-MQ8 (500uM) ${ }^{\mathrm{a}}$ & $1.9 \pm 0.08$ \\
\hline Soluble Fraction & 18'-MQ8 $(1 \mathrm{mM})^{\mathrm{a}}$ & $3.4 \pm 0.5$ \\
\hline Soluble Fraction & 18'-MQ8 $(2 \mathrm{mM})^{\mathrm{a}}$ & $3.6 \pm 0.3$ \\
\hline Soluble Fraction & $18^{\prime}-\mathrm{MQ} 8(5 \mathrm{mM})^{\mathrm{a}}$ & $0.7 \pm 0.1$ \\
\hline
\end{tabular}

${ }^{1}$ pMMO Specific Activity stated in units of $\mathrm{nmol} /$ minute/mg total protein \pm S.E.M.

${ }^{\mathrm{a}} 5 \mathrm{mM}$ NADH was added as the reductant in these samples. 
diluted by membrane solubilization, leading to a decrease in pMMO activity. With this in mind, the experiments in Table 1 were performed in a similar fashion to those in Chapter 3 to determine the effects of $18^{\prime}$-methylene $\mathrm{CoQ}_{8}$ on pMMO activity as compared to previously published exogenous quinones.

Relative to formate and the two exogenous quinols (Table 1), the reduced form of 18 'methylene $\mathrm{CoQ}_{8}$ performed poorly in vivo. At low concentrations of 18 '-MQ8 $\mathrm{H}_{2}$, pMMO exhibited only a small portion of the activity observed with formate or the exogenous quinols. Furthermore, the pMMO activity actually decreased with increasing 18 '-MQ8 concentration, to the point of being undetectable at a concentration of $1 \mathrm{mM}$ quinol.

The results for the membrane fraction were similar. At low concentrations, 18'methylene $\mathrm{CoQ}_{8}$ supported low levels of pMMO activity. In this case, however, the activity increased with increasing quinol concentration, ultimately reaching a level comparable to that observed with $5 \mathrm{mM}$ decyl-plastoquinol. Duroquinol outperformed 18 '-MQ8 $\mathrm{H}_{2}$ as a reductant in the membrane fraction, supporting $\sim 5$-fold greater pMMO activity.

The endogenous quinone gave the most promising results when used as a reductant for the solubilized form of pMMO. Here, pMMO specific activity supported by 18 '-methylene $\mathrm{CoQ}_{8}$ attained a rate approximately three quarters of that observed in the presence of decyl-plastoquinol. The maximum rate was observed with $2 \mathrm{mM}$ endogenous quinone and was significantly inhibited at higher concentrations of the quinone (Table 1). Interestingly, this inhibition has previously been observed with duroquinol but not decyl-plastoquinol. 


\section{DISCUSSION}

The respiratory chain of Methylococcus capsulatus remains poorly characterized. The experiments described above were developed to identify an exogenous quinone(s) which may serve as a vehicle for electron transfer reactions within the membrane of the bacteria. It had previously been shown that pMMO activity could be retained following solubilization with lauryl maltoside only if quinones were present to provide reducing equivalents (18). Additionally, a novel quinone was isolated and identified by Green and Collins (4) raising the possibility that this quinone could serve as a source of reducing equivalents for pMMO, as is common in many bacterial respiratory chains $(1,2,8)$. We are also interested in potentially utilizing the endogenous quinone as a reducing source during future purifications of the enzyme.

In addition to the quinone previously described by Collins and Green (18'methylene $\mathrm{CoQ}_{8}$ ) we have identified two other quinones within the membrane fraction of M. capsulatus which had not previously been described; namely $\mathrm{CoQ}_{9}$ and 18 '-methylene $\mathrm{CoQ}_{9}$. All quinones identified from the $n$-pentane extract in this study were ubiquinones. Neither plastoquinones nor menaquinones were isolated. This is consistent with previous studies on the quinone content of methanotrophs $(5,21)$. The difference between our results and those of Collins and Green could be attributed to either differences in the solvent/purification scheme employed or the difference in growth conditions. The previous report detailed growth on a methane:air ratio of 50:50 at $30^{\circ} \mathrm{C}$ for 7 days. In our laboratory, we typically use a methane:air ratio of $20: 80$ at $42^{\circ}-45^{\circ} \mathrm{C}$ for a total period of 2-3 days in the fermentor. It has been reported that bacterial lipid content will vary depending on growth temperature (10). 
The endogenous quinone, 18'-methylene $\mathrm{CoQ}_{8}$, was the focal point of reconstitution studies due to the high amount present in the extract relative to the other endogenous quinones. Initial reconstitution experiments were attempted as an adaptation of the method by Kurreck, et al. (12) in which the whole cell or membrane fraction is sonicated in the presence of added quinol (reduced with sodium dithionite/sodium borohydride in acidic methanol). However, no activity was observed following use of this method (data not shown).

Alternatively, the quinone was reduced and solvent evaporated to dryness under vacuum and reincorporation was performed by gentle mixing with membranes as previously reported for the detergent soluble fraction (Chapter 3). The results from this method also resulted in very low pMMO specific activity (Table 2). However, it must be noted that the quinol is extremely sensitive to oxidation since much of the quinol reverted to the yellow color indicative of the oxidized quinone prior to addition of the enzyme sample. In part, this instability may explain the failure of the added quinol form of 18methylene $\mathrm{CoQ}_{8}$ to support pMMO activity. Originally, it was thought that the greater activity of duroquinol and decyl-plastoquinol resulted from their similarity in structure and redox potential to quinols that would reduce pMMO in vivo.

The results of similar experiments with the membrane fraction did not show much promise, either. Again, only minimal activity was observed with the reduced form of the endogenous quinol over the concentration range specified (Table 1).

The results for the detergent-solubilized membrane fraction, however, differ greatly from those for the whole cell and membrane suspensions along with NADH. In this case, the endogenous quinone was added in its oxidized form to allow the 
endogenous reducing system to perform the task of quinone reduction. Following reincorporation of the endogenous quinone within the solubilized membrane fraction, pMMO specific activity increased with increasing quinone concentration then became inhibited at relatively high amounts $(5 \mathrm{mM})$. Addition of the reduced form of the endogenous quinol to the detergent solubilized fraction gave results similar to those with whole cells and membranes. This suggests that the instability of the synthetically reduced endogenous quinol could be responsible for its inability to reduce pMMO in the whole cell and membrane suspension samples.

The specific activity attained with the endogenous quinone is approximately three quarters of that attained with the reduced decyl-plastoquinol and higher than that of exogenous duroquinol (Table 1). It seems that the enzymatic activity of pMMO is unaffected by the endogenous quinol until relatively high concentrations are achieved. Analogous results have been observed with duroquinol and may be evidence of competitive inhibition by the oxidized form of the quinone. It has been reported that the conformation of the isoprenyl chain relative to the semiquinone head may play a role in the selectivity for quinones in the photosynthetic enzymes of bacteria and higher plants (22). Solubilization of pMMO by lauryl maltoside may result in conformational changes to pMMO that render it more favorable for plastoquinol binding than for ubiquinol binding. Zheng, et al. reported that the isoprenyl $\mathrm{C} \beta C \gamma$ bond is coplanar with the semiquinone ring in plastoquinone while that for ubiquinones is at a $90^{\circ}$ angle with respect to the ring (22). Since duroquinol has no such C-C bond, it is conceivable that a conformationally-altered pMMO would recognize it as being similar to decylplastoquinol, with the "tail" (methyl group) being detected as planar. It seems plausible 
that this sort of steric hindrance may play a role in the relative inability of ubiquinol analogs to reduce solubilized pMMO in vitro.

Results with the oxidized 18 '-methylene $\mathrm{CoQ}_{8}$ suggest addition of this cofactor can overcome loss of quinone cofactors that occurs following solubilization. It appears at least possible that a ubiquinone could be the mobile electron carrier responsible for the reduction of pMMO. This is further substantiated by the fact that neither plastoquinone nor menaquinone were observed in the $n$-pentane extract from the lyophilized membrane fraction. Thus, it appears that the reduction of pMMO by exogenous quinols such as decyl-plastoquinol and duroquinol is adventitious in nature. Their high relative hydrophobicity may enhance their ability to access the pMMO active site or surrounding cofactors, resulting in non-specific reduction.

In conclusion, we have shown that an endogenous quinol present in high amounts in $M$. capsulatus membranes can support solubilized pMMO specific activity in vitro, suggesting that this cofactor may also be responsible for in vivo reduction, as well. However, the data also suggest that it is not a good candidate for pMMO reduction during purification due to its apparent ease of auto-oxidation in comparison to that of both decylplastoquinol and duroquinol. One of the latter two quinols (preferably decylplastoquinol) still appears to be a better candidate for screening of pMMO activity during future purifications as these quinols can be crystallized, precipitated and stored in reduced form. This is not the case for the endogenous ubiquinol. Additionally, the latter two are available commercially whereas 18 '-methylene $\mathrm{CoQ}_{8}$ is not.

This data suggests that we change our present model of pMMO reduction in vivo. It was previously hypothesized that a plastoquinol served as the reductant in vivo. 
However, the absence of any such quinones following organic solvent extraction as well as the results obtained with 18 '-methylene $\mathrm{CoQ}_{8}$ make us question this hypothesis. The ubiquinone isolated here is a potential reductant, yet it requires specific conditions in which to exert its action. It is still hypothesized that a dehydrogenase such as that reported by Cook (6) reduces a quinone (likely 18 '-methylene $\mathrm{CoQ}_{8}$ ) and these reducing equivalents are subsequently transferred to $\mathrm{pMMO}$, most likely in a direct fashion. However, it must be noted that no experimental evidence for NDH-2-mediated reduction of $18^{\prime}$-methylene $\mathrm{CoQ}_{8}$ exists at this time. Therefore, the earlier results by Smith and Dalton (19) may still be explained by the hypothesis that lauryl maltoside either disrupts a reductase to pMMO transfer or dilutes the quinone pool in a fashion which is overcome by addition of quinone/quinol.

\section{ACKNOWLEDGEMENTS}

We would like to thank Mr. Bob Smith, Biochemistry Core Facility, for his analytical expertise in the analysis of samples via GC/MS and Dr. Peter Gannett, WVU School of Pharmacy, for his invaluable assistance producing all Nuclear Magnetic Resonance spectra. 


\section{REFERENCES}

1. Ackrell, B.A.C. and Colin W. Jones. 1971. The respiratory system of Azotobacter vinelandii. Eur. J. Biochem. 20:22-28.

2. Anraku, Y. 1988. Bacterial electron transport chains. Ann. Rev. Biochem. 57:101-132.

3. Anthony, C. 1986. Bacterial oxidation of methane and methanol. Adv. Microb. Physiol. 27:113-209.

4. Collins, M.D. and P.N. Green. 1985. Isolation and characterization of a novel coenzyme Q from some methane-oxidizing bacteria. Biochem. Biophys. Res. Comm. 133:1125-1131.

5. Collins, M.D., O.W. Howarth, and P.N. Green. 1986. Arch. Microbiol. 146:263-266.

6. Cook, S.A. and A.K. Shiemke. 1996. Submitted to Journal of Bacteriology.

7. DiSpirito, A.A., A.K. Shiemke, S.W. Jordan, J.A. Zahn, and C.L. Krema. Cytochrome $a a_{3}$ and cytochrome $a a_{3}$-cytochrome $c_{557}$ complex from Methylococcus capsulatus.

8. Drabikowska, A.K. 1977. The respiratory chain of a newly isolated Methylomonas P11. Biochem. J. 168:171-178.

9. Duine, J.A. 1991. Quinoproteins: enzymes containing the quinone cofactor pyrrolo-quinoline quinone, topaquinone or tryptophan tryptophan quinone. Eur.J. Biochem. 200:271-284. 
10. Jahnke, L.L. 1992. The effects of growth temperature on the methyl sterol and phospholipid fatty acid composition of Methylococcus capsulatus (Bath). FEMS Microbiol. Lett. 93:209-212.

11. Jones, C.W. and E.R. Redfearn. 1966. Biochim. Biophys. Acta. 113:467-481.

12. Kurreck, J., A.G. Seeliger, F. Reifarth, M. Karge, and G. Renger. 1995. Reconstitution of the endogenous quinone pool in photosystem II (PSII) membrane fragments, inside-out vesicles, and PSII core complexes from spinach. Biochemistry 34:15721-15731.

13. Lenaz, G., A, DeSantis, and E. Bertoli. 1985. In Coenzyme Q (G. Lenaz, ed.). Wiley, Chichester, U.K.

14. Moore, H.W., and K. Folkers. 1968. J. Am. Chem. Soc. 88:567-570.

15. Nguyen, H.-H.T., S.J. Elliott, J.H.-K. Yip, and S.T. Chan. 1997. J. Biol. Chem. 273:7957-7966.

16. Redfearn, E.R. 1966. Vitam. Horm. 24:465-488

17. Rich, P.R. 1981. Biochem. Biophys. Acta. 637:28-33.

18. Shiemke, A.K., S.A. Cook, T. Miley, and P. Singleton. 1995. Detergent solubilization of membrane-bound methane monooxygenase requires plastoquinol analogs as electron donors. Arch. Biochem. Biophys. 321:421-428.

19. Smith, D.D.S. and H. Dalton. 1981. In The Prokaryotes. M. Starr (Ed.). Springer-Verlag, New York.

20. Trumpower, B.L. 1990. The proton-motive Q cycle: energy transduction by coupling of proton translocation to electron transfer by the cytochrome $b c_{1}$ complex. J. Biol. Chem. 265:11409-11412. 
21. Urakami, T. and K. Komagata. 1986. J. Gen. Microbiol. 32:317-341.

22. Zheng, M. and G.C. Dismukes. 1996. The conformation of the isoprenyl chain relative to the semiquinone head in the primary electron acceptor $\left(\mathrm{Q}_{\mathrm{a}}\right)$ of higher plant PSII (plastosemiquinone) differs from that in bacterial reaction centers (ubisemiquinone or menasemiquinone) by ca. $90^{\circ}$. 


\section{CHAPTER FIVE}

Oxygen Uptake Studies and Spectroscopic Kinetics 


\section{INTRODUCTION}

Oxidative phosphorylation is an important process in aerobic organisms, allowing the organisms to harness energy by coupling oxidation of reduced cofactors to phosphorylation of ADP, thereby producing an "energy currency" for individual cells. The study of bacterial oxidative phosphorylation has required the development of cellfree systems due to the impermeability of the outer membrane. Unlike eukaryotic systems, electron transport chains in bacteria are diverse due to the fact that there are many different sources and acceptors of electrons used by the various organisms. Additionally, the protein components can differ between organisms and, in some cases, within the same organism, depending on growth conditions $(2,5,6)$

The key carbon component which undergoes oxidation for the release of energy in M. capsulatus is methane. The large amount of energy available upon oxidation of methane is harnessed via an electron transport chain in methylotrophic bacteria (3). These electron transport chains consist of dehydrogenases, iron-sulfur proteins, quinones, cytochromes and cytochrome oxidases. Little is known concerning the Fe-S proteins and all quinones identified, to date, have been ubiquinones $(12,24)$.

Among the aerobic methylotrophs, the chains do not appear to differ to a great extent in the nature of components. The three key differences are the presence or absence of cytochrome $c$, the nature of the terminal oxidase(s) (type $a a_{3}$ or $o$ ), and the branch point to the alternative oxidases (2). Another critical aspect of the methylotrophic bacteria which must be kept in mind concerns methane oxidation: every molecule of growth substrate (methane, in the case of $M$. capsulatus) is oxidized by way of methanol dehydrogenase, including those that are eventually assimilated into cellular carbon. This 
suggests the possibility that there could be an electron transport chain involved in methanol oxidation that is separate from that involved in the oxidation of NADH (5). Therefore, the knowledge gained with regards to methylotrophs will serve as a foundation from which to approach our discussion of the methanotrophic M. capsulatus. These ideas, in addition to what has been learned concerning eukaryotic mitochondria, will form a basis for comparison of respiratory chain proteins.

In this chapter, we will turn our attention to proteins typically found in the latter portions of respiratory chains. In particular, we investigated the function of the periplasmic methanol dehydrogenase, cytochrome $c$, and terminal oxidases. Additionally, with regards to the apparent cytochrome $b$ content of $M$. capsulatus, we will investigate the possibility of the existence of a $b c_{1}$-like complex.

Our initial model suggested that reducing equivalents from methanol entered the respiratory chain of $M$. capsulatus at the level of cytochrome $c$. This particular point was hypothesized by Grozdev, et al (1983) and subsequently shown to occur by Anthony, et al. (4). Methylococcus capsulatus has been shown to grow on both methane and methanol, albeit at much slower rates when grown on the latter (25). Therefore, it is of interest to investigate this portion of the putative electron transport chain to either support or refute this argument.

Also, proteins such as cytochrome $c(8,14)$ and terminal oxidases have been shown to be present in $M$. capsulatus (11), so the enzymatic activity of these enzymes would be interesting to investigate, as well. Our model Odepicts reducing equivalents passing from a cytochrome $c$ (received from either a $b c_{1}$-like complex or methanol dehydrogenase) to a terminal oxidase (most likely as that described by DiSpirito, et al.). 


\section{RESULTS}

The results in Table 1 detail the percent inhibition of oxygen uptake by several well-characterized inhibitors of respiratory chains in other organisms. The inhibition of the background rates was measured with no added reductant. As illustrated in Table 1, the respiratory inhibitors piericidin A, amytal, rhein, HQNO, caffeine, myxothiazole, antimycin A, and SHAM had no effect on the rate of oxygen uptake in membranes or whole cells, regardless of the reductant. These inhibitors were not used for further study.

The results observed for respiratory inhibition were very different when potassium cyanide, octyl gallate, or both were used to inhibit oxygen uptake (Table1). When analyzing the membrane fraction, cyanide, octyl gallate and the combination proved to be inhibitory to oxygen uptake when background respiration was measured. This background respiration is presumably dependent on endogenous substrates. Each inhibitor inhibited approximately half of the oxygen uptake when used alone while the combination inhibited $>90 \%$. When the exogenous reductant NADH was added to the crude membrane preparation, each inhibitor again inhibited oxygen uptake by approximately one-half while the combination abolished nearly all (95.4\%) respiration (Table 1). When both inhibitors were used, the order of addition of inhibitor (cyanide vs. octyl gallate), made no significant difference in the total amount of inhibition observed (data not shown).

On the other hand, the results of inhibition by cyanide and octyl gallate were different when analyzing the whole cell suspension (Table 1). Background respiration was completely abolished when either of the two inhibitors were used and identical results were obtained when methanol was added as the exogenous substrate. A small 
Table 1. Effects of respiratory chain inhibitors on oxygen uptake in Methylococcus capsulatus whole cells and membranes.

\begin{tabular}{|c|c|c|c|c|c|}
\hline & \multicolumn{2}{|c|}{ MEMBRANES } & \multicolumn{3}{|c|}{ WHOLE CELLS } \\
\hline & basal $^{\mathbf{a}}$ & + NADH & basal $^{\mathbf{a}}$ & + Formate & $+\mathrm{MeOH}$ \\
\hline Piericidin A $(500 \mu \mathrm{M})$ & 0 & 0 & 0 & 0 & 0 \\
\hline Amytal $(500 \mu \mathrm{M})$ & 0 & 0 & 0 & 0 & 0 \\
\hline Rhein (500uM) & 0 & 0 & 0 & 0 & 0 \\
\hline $\mathrm{HQNO}^{\mathrm{b}}(1 \mathrm{mM})$ & 0 & 0 & 0 & 0 & 0 \\
\hline Caffeine (1mM) & 0 & 0 & 0 & 0 & 0 \\
\hline Myxothiazole $(500 \mu \mathrm{M})$ & 0 & 0 & 0 & 0 & 0 \\
\hline Antimycin A $(500 \mu \mathrm{M})$ & 0 & 0 & 0 & 0 & 0 \\
\hline SHAM $(3.5 \mathrm{mM})$ & 0 & 0 & 0 & 0 & 0 \\
\hline Cyanide $(500 \mu \mathrm{M})$ & $52 \%^{\mathrm{a}}$ & $58.4 \pm 1.9$ & 100 & $96.1 \pm 0.7$ & 100 \\
\hline Octyl Gallate $(500 \mu \mathrm{M})$ & $58 \%{ }^{\mathrm{a}}$ & $56.2 \pm 1.5$ & 100 & 100 & 100 \\
\hline $\mathrm{OG}+\mathrm{CN}^{-}(500 \mu \mathrm{M}$, each $)$ & $93 \%^{\mathrm{a}}$ & $95.4 \pm 0.8$ & n.d. & n.d. & n.d. \\
\hline
\end{tabular}

${ }^{\mathrm{a}}$ Background respiration rate measured without added reductant.

${ }^{\mathrm{b}} \mathrm{HQNO}=2 n$-heptyl-4-hydroxy-quinoline-N-oxide 
amount of residual activity remained when cyanide was used to inhibit oxygen uptake with formate as the source of reducing equivalents, whereas all activity was abolished in this system when octyl gallate was utilized (Table 1).

Oxygen uptake in the detergent-solubilized membrane fraction was observed with NADH as reductant. This was performed in an analogous manner to studies with detergent-solubilized pMMO where quinone concentration of the system was supplemented by an addition of exogenous quinone $\left(\mathrm{Q}_{0}\right)$. The data in Figure 1 depict the oxygen uptake activity of a typical solubilized membrane preparation reduced with $\mathrm{NADH}$ and supplemented with exogenous quinone. The oxygen uptake activity was minimal in the presence of NADH alone, but was dramatically enhanced following the addition of the quinone in vitro.

In view of the interesting results obtained with the solubilized fraction, a similar investigation of the unsolubilized membrane fraction was initiated. The reduced forms of the exogenous quinones $\mathrm{Q}_{0}$ and $\mathrm{Q}_{2}$ as well as the purified, endogenous 18'-methylene $\mathrm{CoQ}_{8}$ were examined for their ability to increase oxygen uptake in the crude membrane fraction. All three quinols increased the rate of oxygen consumption above the basal rate observed in membranes alone (data not shown). The endogenous quinol had the smallest effect of the three. These oxygen uptake rates were about one-third that observed in the solubilized fraction with $\mathrm{Q}_{0}$. Octyl gallate inhibited oxygen uptake $~ 70-80 \%$ (Table 2) while cyanide inhibited approximately 20-35\% (Table 2) irrespective of the nature of the quinol.

Reduction of the crude membrane fraction by the reduced form of cytochrome $c$ (from horse heart) increases oxygen uptake, as well (Figure 2). 500 $\mu \mathrm{M}$ cyanide inhibited 


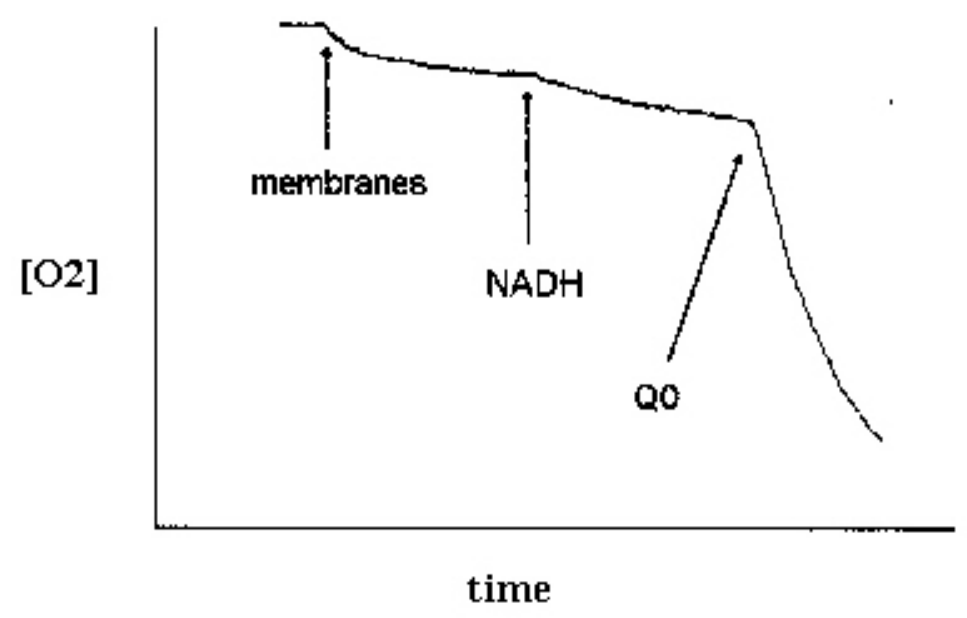

Figure 1. $\mathrm{Q}_{0}$ stimulates oxygen uptake in the solubilized membrane fraction in the presence of NADH. 
Table 2. Percent Inhibition of respiration in the membrane fraction by cyanide and octyl gallate in the presence of exogenous and endogenous quinols.

\begin{tabular}{|c|c|c|c|}
\hline & $\mathrm{Q}_{0} \mathrm{H}_{2}$ & $\mathrm{Q}_{2} \mathrm{H}_{2}$ & $18^{\prime}$-methylene $\mathrm{CoQ}_{8} \mathrm{H}_{2}$ \\
\hline Cyanide & $21 \%$ & $28 \%$ & $35 \%$ \\
\hline Octyl Gallate & $79 \%$ & $70 \%$ & $78 \%$ \\
\hline
\end{tabular}



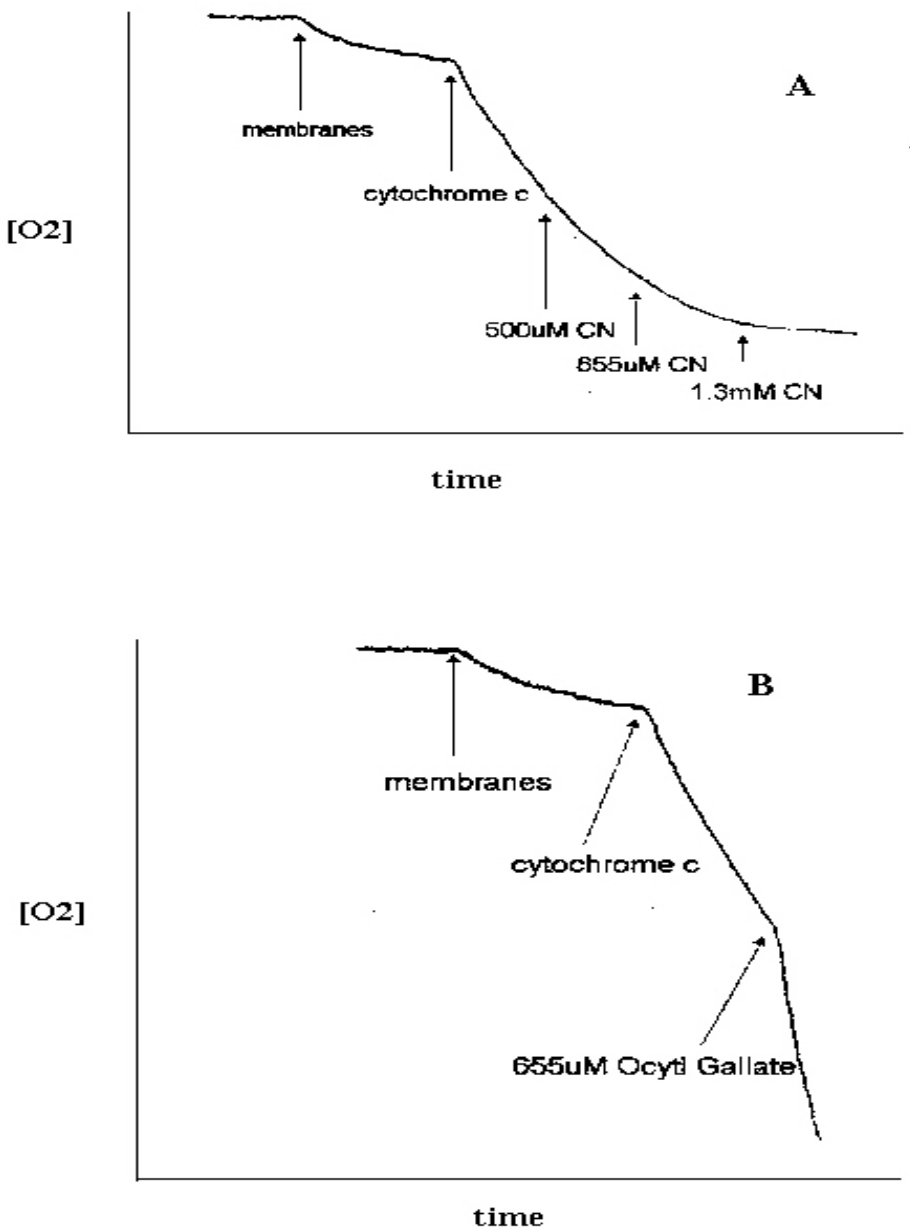

Figure 2. Reduced cytochrome $c$ (horse heart) stimulates oxygen uptake in the membrane fraction. Cyanide inhibition detailed in A, octyl gallate effects shown in B. 
$57 \%$ of this rate, $655 \mu \mathrm{M}$ cyanide inhibited $72 \%$ and $1.3 \mathrm{mM}$ cyanide inhibited $93 \%$ (Figure 2A). Surprisingly, however, octyl gallate (in DMSO) actually increases this rate by approximately 2 -fold (Figure 2B).

To study the reduction of endogenous cytochrome $c$, the difference spectrum (dithionite reduced - air oxidized) was established for isolated membranes (Figure 3). The signal to noise ratio of the analogous spectrum in whole cells was unacceptable (data not shown), so the reduction of cytochrome $c_{555}$ was investigated only in the membrane fraction. As illustrated in Figure 4A, an increase in cytochrome $c_{555}$ reduction was observed when a solution of octyl gallate was added in methanol. No increase in absorbance was observed when the membrane fraction was boiled prior to analysis (baseline trace, Figure 4A). The initial velocity of the reaction increased with increasing substrate concentration. Additionally, no increase was observed with methanol alone or when the inhibitor/substrate mixture was added to buffer in the absence of membranes (data not shown). Similarly, when an aliquot of methanol was added to the same preparation of membranes, an increase in cytochrome $c_{555}$ reduction was observed in the presence of cyanide (Figure 4B). However, the rate of this increase was dramatically slower than that in the presence of octyl gallate (Figure 4). Again, no increase in absorbance was observed with a boiled membrane preparation (data not shown).

To ascertain the response of exogenous cytochrome $c$, the octyl gallate/methanol solution was added to membrane preparations with varying amounts of horse heart cytochrome $c$. The amplitude of the response increased with increasing amounts of cytochrome $c$, however, the initial rate of reduction was unaffected (Figure 5). 


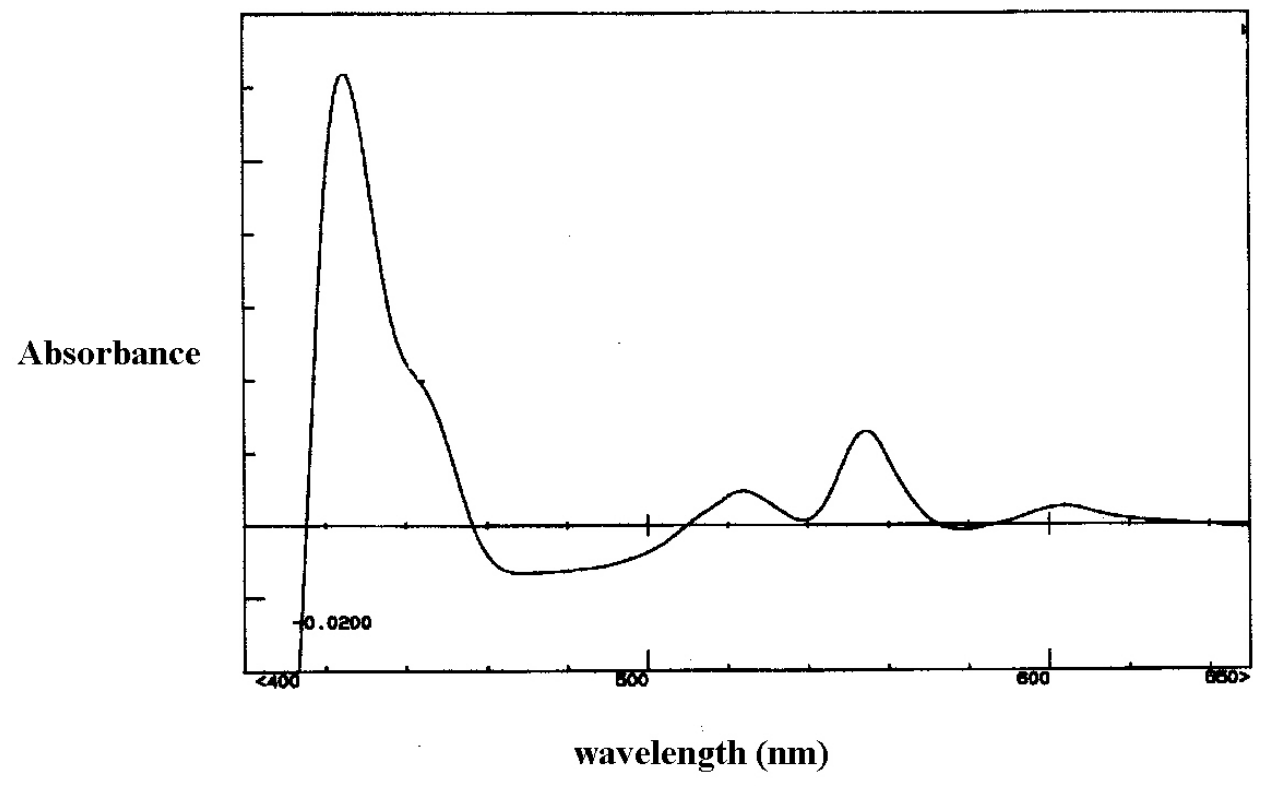

Figure 3. Dithionite-reduced minus air-oxidized difference spectrum for M. capsulatus membrane fraction. Note the absorbance at $555 \mathrm{~nm}$, indicative of cytochrome $c$. 

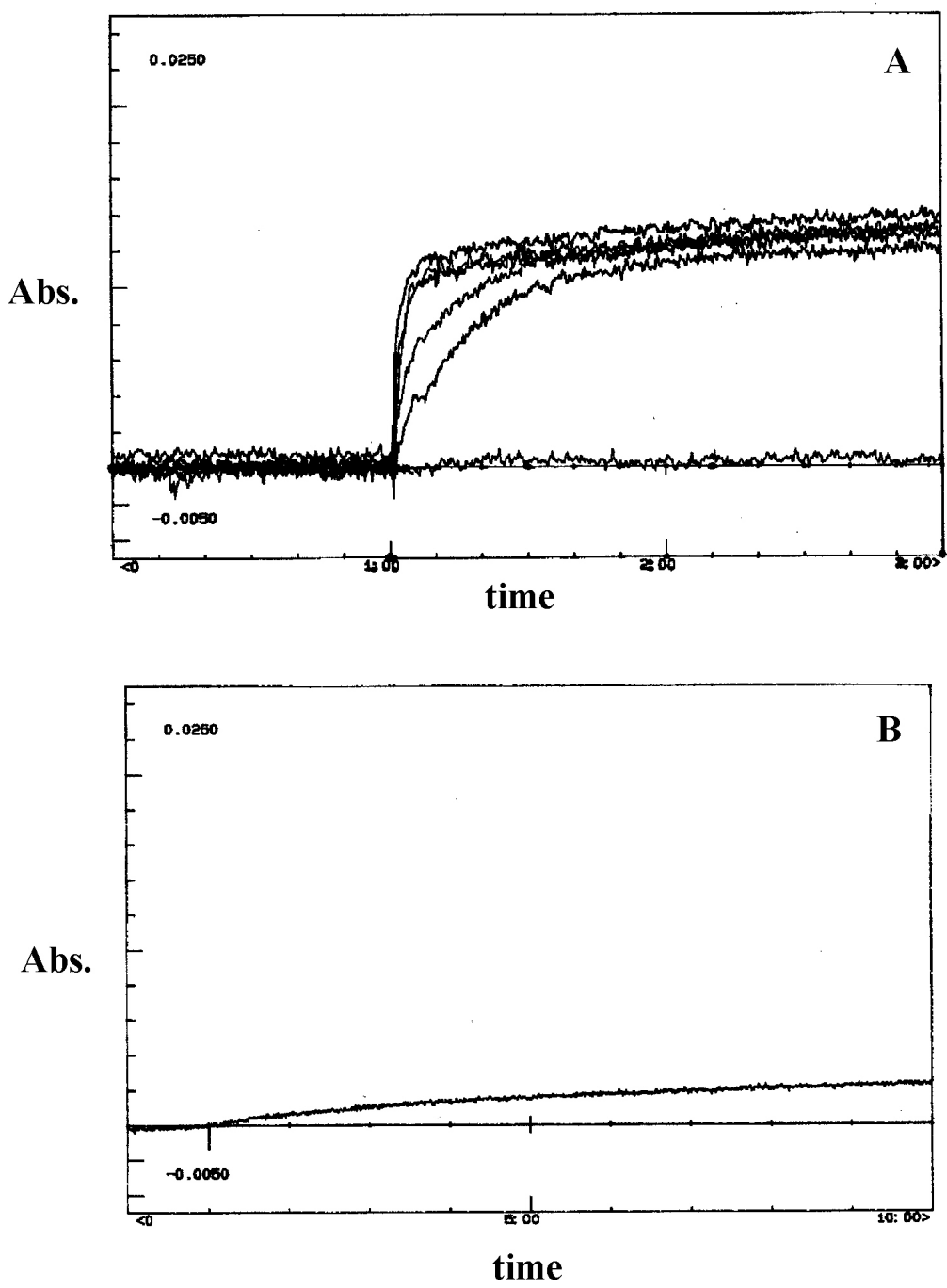

Figure 4. A) Increasing amounts of methanol $(0.1 \mu \mathrm{L}, 0.2 \mu \mathrm{L}, 0.3 \mu \mathrm{L}, 0.4 \mu \mathrm{L}, 0.5 \mu \mathrm{L})$ coincide with increased rates of cytochrome $c_{555}$ reduction in membranes as measured by increased abs @555nm. Octyl gallate was included to prevent oxidation of cytochrome $c$, thus allowing visualization of the absorbance increase. The baseline trace is a result of the addition to boiled membrane fraction. B) Addition of methanol $(0.5 \mu \mathrm{L})$ to membranes pre-incubated with potassium cyanide results in cytochrome $c$ reduction at a slower rate. 


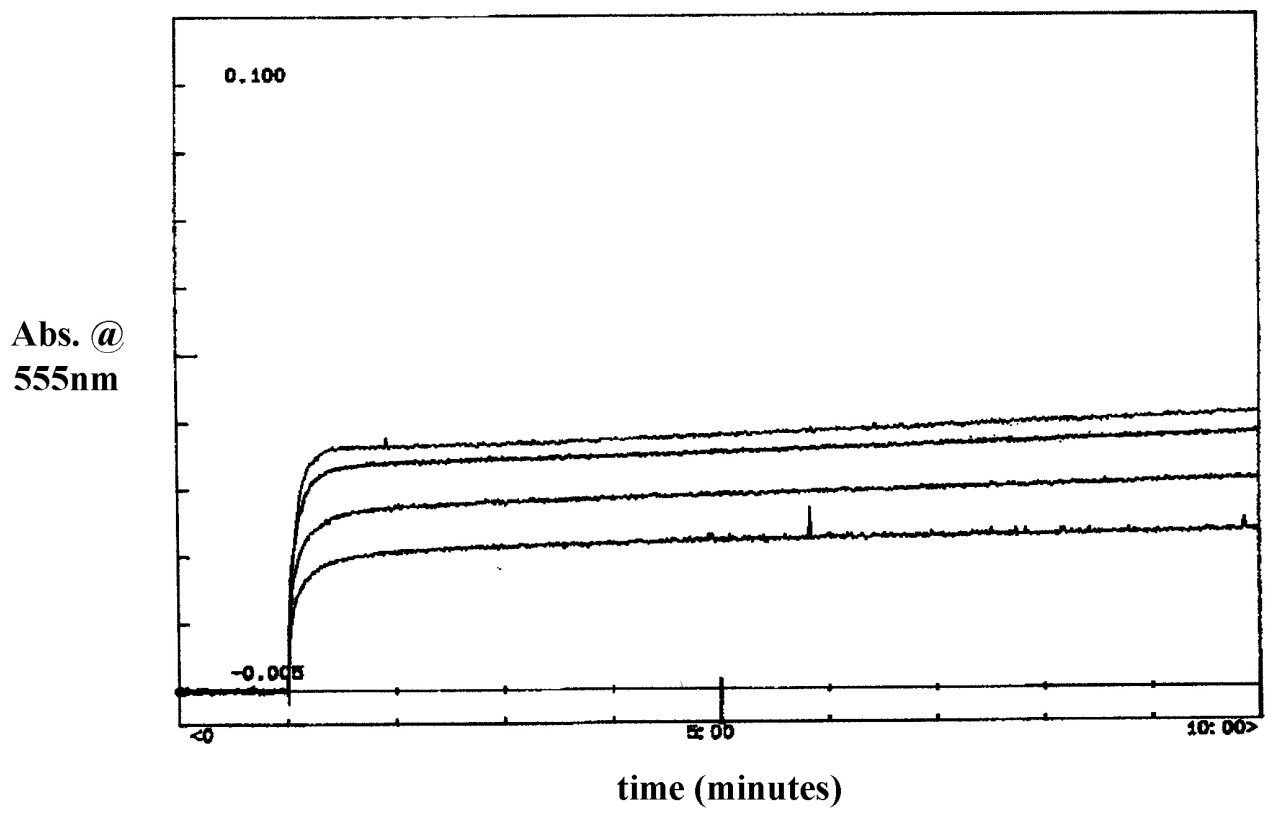

Figure 5. Amplitude of absorbance at $555 \mathrm{~nm}$ is a directly proportional to added exogenous cytochrome $c$ (horse heart). Addition of octyl gallate in methanol at 1 minute. 
Finally, to examine the ability of quinols to reduce the endogenous cytochrome $c_{555}$, an aqueous solution of $\mathrm{Q}_{0} \mathrm{H}_{2}$ was added to a membrane preparation which had been preincubated with solid octyl gallate. As Figure 6 shows, a minimal increase in absorption is observed when the quinol is used as a source of electrons with octyl gallate used as inhibitor. Similar results were obtained when the quinol was added to a membrane preparation which had been preincubated with cyanide (data not shown). A range of quinol concentrations $(50 \mu \mathrm{M}-1.0 \mathrm{mM})$ were used, with no observable cytochrome $c$ reduction in any case. 


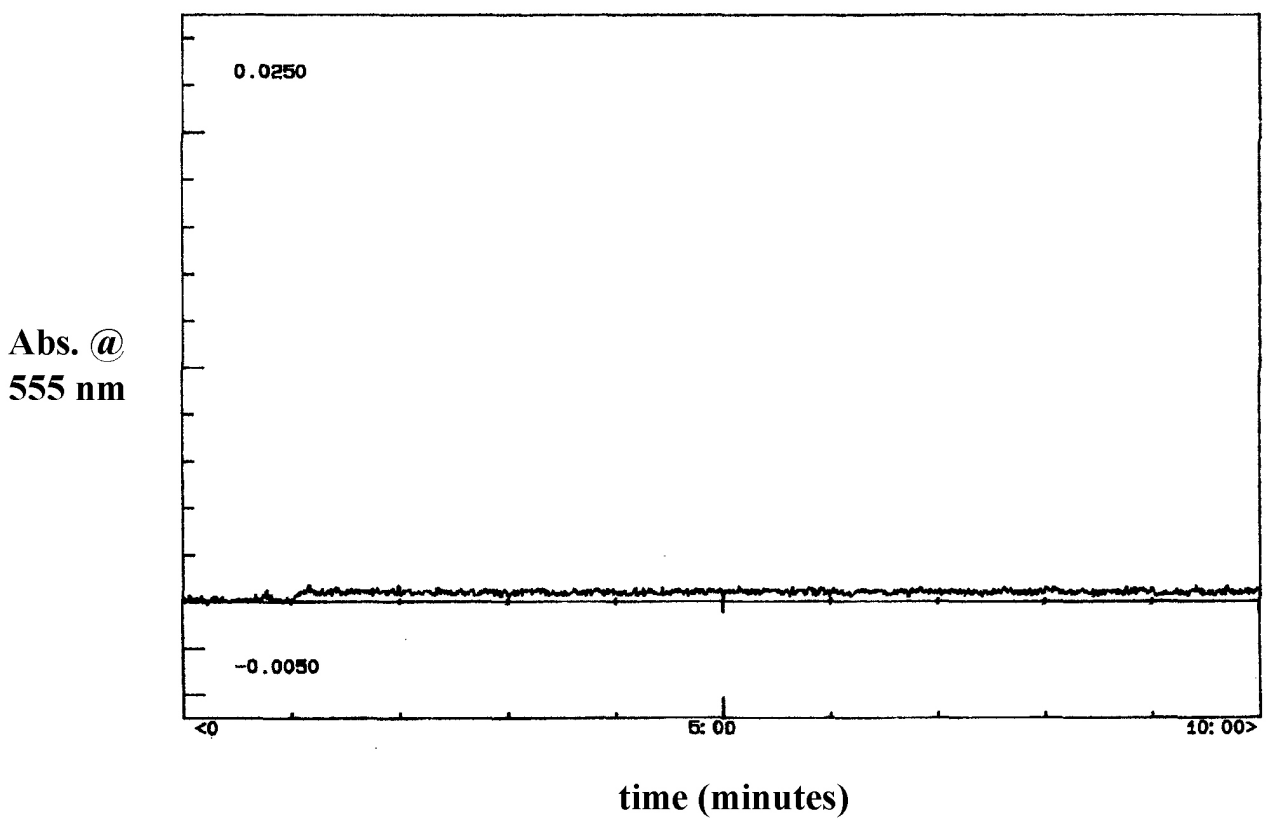

Figure 6. Addition of $250 \mu \mathrm{M} \mathrm{Q}{ }_{0} \mathrm{H}_{2}$ to the membrane fraction does not result in cytochrome $c$ reduction, in the presence of octyl gallate. 


\section{DISCUSSION}

The data in Table 1 illustrate the effects of a variety of respiratory inhibitors on oxygen consumption in both the membrane fraction (in vitro) as well as the whole cell suspension (in vivo). Most of the inhibitors had no effect. For instance, additions of rhein (17) and amytal $(10,21)$ to respiring preparations (either membranes or whole cells) exhibited no inhibition of oxygen uptake. The data for amytal is somewhat inconsistent with the results previously reported by Cook in which a $\sim 50 \%$ decrease in NDH-2 activity was observed with additions of $250 \mu \mathrm{M}-1.0 \mathrm{mM}$ amytal (Cook, Ph.D. dissertation). However, those assays were performed on the purified dehydrogenase with $\mathrm{Q}_{0}$ as the electron acceptor. The $\mathrm{NAD}^{+}$-linked dehydrogenase purified was described as being mildly sensitive to amytal, however, no indication of that is observed here. The previous report mentioned that the amytal binding may have been non-specific (in view of the high concentrations needed for inhibition), and therefore, physiologically irrelevant. Alternatively, there may be another path for electrons from either NADH and/or formate (in whole cells) to oxygen, which is insensitive to amytal. However, from the data presented in this manuscript, it appears unlikely that a type 1 dehydrogenase exists in M. capsulatus.

Also listed in Table 1 are the effects of caffeine and HQNO (21). Neither of these compounds affected respiration either. One of the most potent inhibitors of type-1 NADH dehydrogenases, piericidin A $(10,21)$, was also unable to inhibit oxygen uptake in the two systems studied. Typically, piericidin A need only be used at $\mathrm{nM}$ to $\mathrm{pM}$ concentrations for complete inhibition in eukaryotic mitochondria. Thus, this evidence, along with evidence from the other NADH dehydrogenase inhibitors suggests that the 
only dehydrogenase found in $M$. capsulatus is of type 2, supporting Cook's previous conclusion.

Likewise, the data in Table 1 suggest that there is no $b c_{1}$-like complex in $M$. capsulatus. In mitochondria, the $b c_{1}$ complex is very sensitive to the inhibitors myxothiazole and antimycin $\mathrm{A}$ at $\mu \mathrm{M}$ to $\mathrm{nM}$ concentrations (21) It is also sensitive to HQNO, although, to a lesser extent (21). Here, no evidence for such a complex is observed since neither antimycin A nor myxothiazole inhibited oxygen uptake in either membranes or whole cells at the concentrations studied. This contradicts previous indications for the presence of such a complex, based on UV-vis absorption indicative of cytochromes $b$. These $b$-type cytochromes may correspond to the cytochromes reportedly bound to pMMO after its purification (29). Thus, our revised model does not include such an enzyme within the respiratory chain.

The inhibitor known as SHAM (salicylhydroxamic acid) is an inhibitor of the alternate oxidase found in some plants, bacteria, and trypanosomes $(7,9,23)$. However, we see no evidence for oxygen uptake inhibition by SHAM within the membrane fraction or the whole cell suspension of M. capsulatus. Apparently, if there is another oxidase in addition to the primary one, it is distinct from the SHAM-sensitive types found in plants, bacteria, and trypanosomes.

Finally, inhibition of oxygen uptake was observed with two different inhibitors: cyanide and octyl gallate. Cyanide is a well-known inhibitor of terminal cytochrome $c$ oxidases while octyl gallate has recently been reported as an inhibitor of the alternate oxidase in plants $(1,15)$. As Table 1 illustrates, either inhibitor was capable of reducing oxygen uptake by approximately one-half (with NADH used as reductant) in the 
membrane fraction, and, when used in combination, the oxygen uptake was essentially abolished (Table 1). Interestingly, the oxygen uptake was inhibited completely in whole cells by either inhibitor. Furthermore, the background respiratory rate was inhibited in vitro and in vivo in manners similar to that observed in the presence of exogenous reductants (NADH, formate, and methanol). This seems to suggest that these exogenous reductants act in manners similar to the endogenous reductants responsible for basal levels of respiration.

It has been shown by Anthony, et al. (4) that methanol dehydrogenase is capable of using methanol to reduce a cytochrome $c_{\mathrm{L}}$ in Methylobacterium extorquens, a methylotroph. A similar event was also hypothesized to exist for methanol dehydrogenase and cytochrome $c_{\mathrm{CO}}$ in $M$. capsulatus (12), and increased oxygen uptake in whole cell suspensions following methanol addition is consistent with such a pathway. This pathway from methanol, through methanol dehydrogenase to cytochrome $c$, and, ultimately a terminal oxidase would likely involve an $a a_{3}$-type oxidase such as that reported by DiSpirito, et al. (11). Such terminal oxidases are well known to be inhibited by cyanide.

The site of inhibition of respiration by octyl gallate is still uncertain. The molecular mechanism of inhibition of the alternate oxidase by octyl gallate is not known. Therefore, it is difficult to know which protein(s) this inhibitor is capable of binding in M. capsulatus. However, the molecular structure of octyl gallate is reminiscent of the quinones, so it may compete with quinone binding sites. However, none of the proteins involved in methanol oxidation (methanol dehydrogenase, cytochrome $c$, cytochrome $c_{557}$ 
- cytochrome $a a_{3}$ complex) have been reported to possess quinone binding sites, so the mechanism of its inhibition of respiration from methanol is still a mystery.

If it competes with quinone binding, octyl gallate could be acting at points between formate and the terminal electron acceptor, possibly with enzymes that obtain reducing equivalents from quinols in vivo. However, as mentioned above, no definitive knowledge of octyl gallate's interaction with proteins have been elucidated at the molecular level. Nonetheless, the data from the membrane fraction (Table 1) suggest that M. capsulatus possesses two independent sites of $\mathrm{O}_{2}$ reduction.

Figure 1 illustrates the increase in oxygen uptake following an addition of oxidized $\mathrm{Q}_{0}$ to detergent-solubilized membrane fractions. This result suggests that (at least) one of the oxygen reducing sites in $M$. capsulatus is capable of utilizing reducing equivalents from quinones.

Table 2 illustrates the respiration of the membrane fraction (no detergent solubilization) in the presence of $\mathrm{Q}_{0} \mathrm{H}_{2}, \mathrm{Q}_{2} \mathrm{H}_{2}$, and 18 '-methylene $\mathrm{CoQ}_{8} \mathrm{H}_{2}$. All three quinols increased oxygen consumption in the membrane fraction, with the endogenous quinol the least effective of the three (data not shown). This result may be due to the instability of the reduced form of 18 '-methylene $\mathrm{CoQ}_{8}$ as described in Chapter 4. Since the quinol-linked respiration is sensitive to octyl gallate, and less so towards cyanide (Table 2), the site of inhibition may be related to the alternate oxidase of higher plants $(1,15)$ which are also sensitive to octyl gallate. However, we note that oxygen uptake is only partially inhibited by either octyl gallate or cyanide when quinols are used as reductants in vivo (Table 2). 
Figure 2 depicts the respiration of the membrane fraction (no detergent solubilization) in the presence of the reduced form of cytochrome $c$. The addition of cytochrome $c$ causes an increase in the oxygen uptake of the membrane preparation and this is partially inhibited by additions of cyanide. Up to $93 \%$ inhibition is achieved with $\mathrm{mM}$ concentrations of cyanide. In contrast, additions of octyl gallate increased the rate of oxygen uptake in the membrane fraction by approximately two-fold (Figure 2). This result suggests two possible explanations: (1) the octyl gallate binds the same oxidase as cyanide, increasing its enzymatic activity in some manner, or (2) octyl gallate binds to another enzyme/protein in a manner which is inhibitory, thereby causing an increase in the flux of reducing equivalents through the cytochrome $c$ oxidase. The former argument seems unlikely since octyl gallate decreases oxygen consumption in vitro and in vivo in the presence of $\mathrm{NADH}$, formate and methanol. The latter argument implies that there are two pathways for reducing equivalents from cytochrome $c$ to oxygen and that the cyanide-sensitive pathway is preferred over the octyl gallate-sensitive pathway in vitro. The results with whole cells presented in Table 1 do not appear to be consistent with the two independent pathways for reducing equivalents to travel from cytochrome $c$ to a terminal oxidase. However, it is possible that these pathways are coupled or linked in some unknown way in whole cells and this coupling is disrupted upon cell lysis. Clearly, this is an area where further research is necessary to clarify these discrepancies.

To examine the effects of specific inhibitors on cytochrome $c$ reduction, difference spectroscopy was utilized to monitor reduction of cytochrome $c_{555}$ as a function of time. The (dithionite reduced - air oxidized) difference spectrum was easily observable for membrane preparations (Figure 3). However, the analogous spectrum for 
the whole cell suspension was unacceptable for kinetic studies due to excessive noise. Therefore, cytochrome $c_{555}$ reduction was examined spectroscopically only in the membrane fraction.

Figure 4 illustrates the effect of increasing concentrations of methanol on the cytochrome $c_{555}$ oxidation state in membrane preparations. This increase in rate of reduction occurred only when active enzyme and substrate were in the presence of octyl gallate. The concentration of octyl gallate was held constant as methanol concentration was increased and the amplitude of the curves ultimately reached approximately the same level though with increasing initial velocity (top panel). This amplitude of the absorbance could be increased as a function of added exogenous cytochrome $c$ (Figure 5), confirming that the absorbance change in Figure 4 is due to reduction of endogenous cytochrome $c$. This data shows that the rate of cytochrome $c_{555}$ reduction is increased by increasing amounts of methanol. Similar results were obtained in the presence of cyanide, but the relative rate of reduction was dramatically slower with equivalent amounts of total protein (Figure 4). Together, these results suggest the oxidation of cytochrome $c$ is inhibited by both octyl gallate and cyanide when methanol is available as a substrate and that cyanide may also be inhibiting the reduction of cytochrome $c$ by methanol as well.

Finally, the effect of quinols on the oxidation state of cytochrome $c$ was examined with the reduced form of $\mathrm{Q}_{0}$ (Figure 6). The results were not indicative of cytochrome $c$ reduction when either octyl gallate or cyanide were used to inhibit electron flow. This result suggests that reducing equivalents from quinols use a different pathway from that involving cytochrome $c$. This also suggests that the oxidase activity observed with the 
oxygen electrode in the presence of reduced quinols stems from activity of a quinol oxidase, which is sensitive to octyl gallate. The quinols would directly reduce such an oxidase, increasing oxygen uptake. Since the oxygen uptake in the membrane fraction in the presence of reduced quinols is preferentially inhibited by octyl gallate, the enzyme may resemble the alternate oxidase found in some higher plants. However, it is distinct from the latter in that it is not sensitive to SHAM. 


\section{REFERENCES}

1. Albury, M.S., P. Dudley, F.Z. Watts, A.L. Moore. 1996. Targeting the plant alternative oxidase protein to Scizosaccharomyces pombe mitochondria confers cyanide-insensitive respiration. J. Biol. Chem. 271:17062-17066.

2. Anraku, Y. 1988. Bacterial electron transport chains. Annu Rev. Biochem. 57:101-132.

3. Anthony, C. 1989. Bacterial oxidation of methane and methanol. Adv. Microb. Physiol. 27:179-183.

4. Anthony, C., and S.L. Dales. 1996. In M.E. Lidstrom and F.R. Tabita (eds.) Microbial Growth on C1 Compounds. The biochemistry of methanol dehydrogenase. Kluwer Academic Publishers, Netherlands.

5. Anthony, C.A. 1988. Bacterial Energy Transduction. Academic Press, London, pp. 1-517.

6. Armitage, J.P. 1992. Behavioral responses in bacteria. Annu. Rev. Physiol. 54:683-714.

7. Beattie, D.S., and M.M. Howton. 1996. The presence of rotenone-sensitive NADH dehydrogenase in the long slender bloodstream and procyclic forms of Trypanosoma brucei brucei.

8. Bergmann, D.J., J.A. Zahn, and A.A. DiSpirito. 1999. High-molecular-mass multi-c-heme cytochromes from Methylococcus capsulatus (Bath). 181:991-997. 
9. Berthold, D.A. 1998. Isolation of mutants of the Arabidopsis thaliana alternative oxidase (ubiquinol: oxygen oxidoreductase) resistant to salicyl hydroxamic acid. Biochim. Biophys. Acta. 1364:73-83.

10. Coles, C.J., D.E. Griffiths, D.W. Hutchinson, and A.J. Sweetman. 1968. Binding of piericidin A, rotenone, and amytal in beef heart mitochondria

11. DiSpirito, A.A., A.K. Shiemke, S.W. Jordan, J.A. Zahn, and C.L. Krema. 199X. Cytochrome $a a_{3}$ and cytochrome $a a_{3}$-cytochrome $c_{557}$ complex from Methylococcus capsulatus (Bath).

12. Drabikowska, A.K. 1977. The respiratory chain of a newly isolated Methylomonas P11. Biochem. J. 168:171-178.

13. Douce, R., and M. Neuberger. 1989. The uniqueness of plant mitochondria. Annu. Rev. Plant. Physiol. 40:371-414.

14. Grozdev, R.I., E.L. Nikonova, A.I. Pilyashenko-Novokhatnyi, E.V. Shushenacheva, A.N. Grigoryan, V.S. Belova, and L.A. Shirokova. 1983. Role of CO-binding cytochrome $c$ in the enzymatic oxidation of methane by the bacterium Methylococcus capsulatus.

15. Hoefnagel, M.H., J.T. Wiskich, S.A. Madgwick, Z. Patterson, N. Oettmeir, and P.R. Rich. 1995. New inhibitors of the ubiquinol oxidase of higher plant mitochondria. Eur. J. Biochem. 233:531-537.

16. Jeng, M., C. Hall, F.L. Crane, N. Takahashi, S. Tamura, and K. Folkers. 1968. Inhibition of mitochondrial electron transport by piericidin A and related compounds. Biochemistry. 7:1311-1322. 
17 Kean, E.A. 1968. Rhein: an inhibitor of mitochondrial oxidations. Archives Biochem. Biophys. 127:528-533.

18. Lidstrom, M. 1991. In A. Balows, H.G. Truper, M. Dwqrkin, W. Hander, and K.H. Schleifer (eds.). The Prokaryotes. $2^{\text {nd }}$ edition. Springer-Verlag, New York.

19. Lomans, B.P., H.J. Op den Camp, A. Pol, C. van der Drift, and G.D. Vogels. 1999. Role of methanogens and other bacteria in degradation of dimethylsulfide and methanethiol in anoxic freshwater sediments. Appl. Environ. Microbiol. 65:2116-2121.

20. Nguyen, H.H., S.J. Elliott, J.H. Yip, and S.I. Chan. 1990. The particulate methane monooxygenase from Methylococcus capsulatus (Bath) is a novel copper-containing three-subunit enzyme. Isolation and characterization. J. Biol. Chem. 273:7957-7966.

21. Singer, T.P. 1979. In Methods in Enzymology. Mitochondrial electron transport inhibitors. Academic Press, London. pp. 454-462.

22. Smith, D.D.S., and H. Dalton. 1989. Solubilisation of methane-monooxygenase from Methylococcus capsulatus (Bath). J. Bacteriol. 178:1018-1029.

23. Taton, M., and A. Rahier. 1996. Plant sterol biosynthesis: identification and characterization of higher plant delta-7 sterol C5(6) desaturase. Arch. Biochem. Biophys. 325:279-288.

24. Urakami, T. and K. Komagata. 1986. Occurrence of isoprenoid compounds in gram negative methanol, methane, and methylamine utilizing bacteria. J. Gen. Microbiol. 32:317-341. 
25. Whittenbury, R., K.C. Phillips, and J.F. Wilkinson. 1970. Enrichment, isolation, and some properties of methane utilizing bacteria. J. Gen. Microbiol. 61:205-218.

26. Yagi, T. 1991. The bacterial NADH-quinone oxidoreductases. J. Bioenerg. Biomem. 23:211-225.

27. Yagi, T. 1993. The bacterial energy-transducing NADH-quinone oxidoreductases. Biochim. Biophys. Acta. 1141:1-17.

28. Zahn, J.A., D.M. Arciero, A.B. Hooper, and A.A. DiSpirito. 1996. Cytochrome c' of Methylococcus capsulatus (Bath). Eur. J. Biochem. 240:684691.

29. Zahn, J.A., and A.A. DiSpirito. 1996. Membrane-associated methane monooxygenase from Methylococcus capsulatus (Bath). J. Bacteriol. 178:10181029.

30. Zahn, J.A., C. Duncan, and A.A. DiSpirito. 1994. Oxidation of hydroxylamine by cytochrome P-460 of the obligate methylotroph Methylococcus capsulatus (Bath). J. Bacteriol. 176:5879-5887. 
CHAPTER SIX

\section{CONCLUSIONS}


This project investigated the respiratory chain of M. capsulatus (Bath) in addition to some of the possible interactions of pMMO within this scheme (Figure 1). Much of what is known about the transfer of reducing equivalents in $M$. capsulatus (Bath) centers on reactions involving pMMO. In our laboratory, as well as others, copper ions have been shown to stimulate pMMO activity both in vivo and in vitro. Originally, it was hypothesized that copper ions were in equilibrium with the aqueous environment and the holoenzyme, resulting in the observed increase in pMMO activity upon addition of copper ion (2). Furthermore, recent reports have indicated that transcription of the $3.3 \mathrm{kB}$ polycistronic mRNA (encoding pMMO) of $M$. capsulatus (Bath) was activated by copper ions. A similar event occurred in the methanotroph M. trichosporium OB3b. Additionally, the gene transcription for the soluble form of the enzyme was repressed in both methanotrophs upon addition of cupric ions. Thus, pMMO activity appears to be regulated by copper both post-transcriptionally and post-translationally (4).

Another question with regards to pMMO is the source of its reducing equivalents in vivo. It was previously assumed that $\mathrm{NADH}$ was the direct reductant of pMMO. However, the decrease in pMMO activity in vitro following solubilization seemed to contradict this theory because activity is poor when NADH is used as the reductant in this system. This could explain why earlier attempts at purifying pMMO have failed. As we have shown in Chapter 3, quinols can serve as electron donors to pMMO whereas NADH is unable to support pMMO activity in the solubilized membrane fraction. We suggested that the quinone pool was diluted upon detergent solubilization, thereby decreasing the rate of quinol-mediated reduction of pMMO. This hypothesis was supported by data which showed that pMMO activity could be recovered, following detergent 
solubilization, if the system were supplemented by exogenous quinols. Furthermore, data by Cook and Shiemke suggest that a type $2 \mathrm{NADH}$ dehydrogenase may be responsible for reducing quinones in vivo, providing a link in the chain responsible for the transport of reducing equivalents from NADH to pMMO. Furthermore, following solubilization, pMMO retained characteristics of the membrane-bound form in that it was still stimulated by copper ion supplementation, inhibited by the chelator EDTA, inactivated by acetylene, and radiolabeled on the $26 \mathrm{kDa}$ polypeptide (2).

Given the possibility that a quinol could serve as an electron donor for pMMO in vivo, we set out to examine the endogenous quinones of $M$. capsulatus (Bath) to determine if they could mimic the action of the exogenous quinols utilized in Chapter 3 (duroquinol and decyl-plastoquinol). We isolated, purified, and characterized quinones similar to those previously reported by Collins \& Green (1985), with the exception that our cultures produced two quinones which had not previously been observed $\left(\mathrm{CoQ}_{9}\right.$ and 18'-methylene $\left.\mathrm{CoQ}_{9}\right)$. The dominant species (18'-methylene $\left.\mathrm{CoQ}_{8}\right)$ was capable of enhancing pMMO activity in solubilized membrane fractions but only when added in the oxidized form in combination with NADH. This data, in combination with the fact that no other type of quinone was identified (nor has previously been observed) suggests that 18 '-methylene $\mathrm{CoQ}_{8}$ is the mobile electron carrier in the membrane of $M$. capsulatus which donates reducing equivalents to $\mathrm{pMMO}$.

Finally, classical inhibitor studies and spectroscopy have allowed us to formulate some hypotheses and/or conclusions with respect to other proteins in the respiratory chain of $M$. capsulatus. First of all, both in vitro and in vivo oxygen uptake studies with the oxygen electrode argue against the existence of either a type-1 $\mathrm{NAD}^{+}$-linked 
dehydrogenase (consistent with Cook's results) or a $b c_{1}$-like complex. Secondly, there appears to be a second (alternate) oxidase activity in vitro (supporting Anthony's previous proposal (1)). This oxidase is distinct from the cyanide-sensitive oxidase and seems to be insensitive to SHAM, yet sensitive to octyl gallate. The octyl gallatesensitive oxidase may obtain reducing equivalents primarily from quinols. Also, there could potentially be more than these two types of oxidases in the respiratory chain, but further studies need to be performed to elucidate this. Additional insight could also be gained from studying the effects of the quinone redox state (quinone:quinol ratio) as well as the effects of keto acids, since some species of bacteria and plants have alternate oxidases sensitive to one or the other of these factors $(5,6)$.

Furthermore, it is apparent that an endogenous $c$-type cytochrome is capable of accepting reducing equivalents (probably from methanol dehydrogenase) when methanol is added as a substrate in vitro and in vivo. Electron flow can be blocked by either octyl gallate or cyanide (to a lesser extent) at a point beyond cytochrome $c$ reduction, suggesting that both inhibitors are capable of inhibiting electron flow to a terminal oxidase. Zahn, et al. (7) alluded to the possibility of a cytochrome $c$ reducing pMMO, however, there is no data to support this possibility. Furthermore, some of the interactions studied in vitro (following cell lysis) may be non-physiological in nature, thus, the in vivo results are more biologically relevant

In the future, several avenues could be explored. Purification of pMMO should probably be explored using exogenous quinols as electron donors (most notably, decylplastoquinol) in solubilized fractions. For the past few years, the research in this laboratory has suggested that quinone pool dilution by detergent could be the key 
shortcoming in past attempts at protein purification. This type of method may also overcome the extreme conditions under which the recent "purifications" had to be performed (anaerobic environments, etc.) $(3,7)$.

Further isolation and purification of the secondary oxidase activity found in vitro could be investigated. It seems odd that an additional activity has been observed in vitro that is not apparent in vivo, so purification of this activity from the membrane fraction, as well as subsequent sequence analysis of the entity, may help clarify the respiratory chain of M. capsulatus (Bath).

Additionally, more detailed investigations of pMMO and/or membrane-bound oxidase interactions with the endogenous quinones could be noteworthy. Resuspension of these proteins in phospholipid vesicles along with the quinol counterparts may lead to insights not practical in crude membrane preparations or detergent-solubilized fractions. This route may also lead to more relevant results in spectroscopic analyses, steering clear from the potential problems with organic solvents mentioned here.

Thus, it appears that a foundation for the respiratory chain of Methylococcus capsulatus (Bath) has been outlined, but much work remains to be performed. 


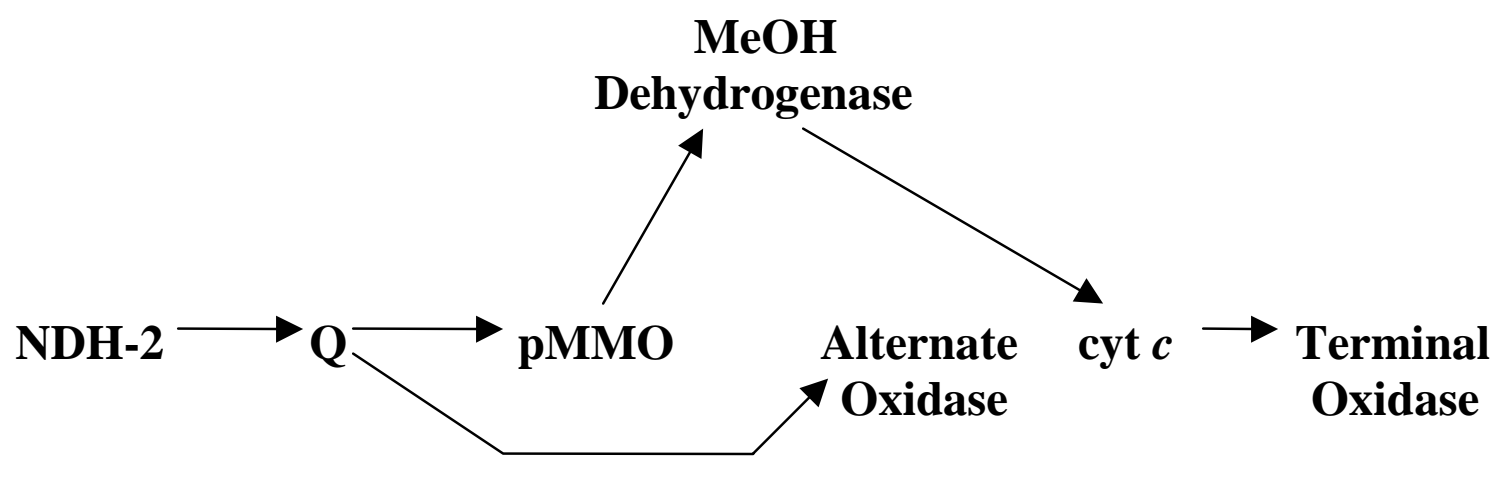

Figure 1. Respiratory Chain Model of M. capsulatus 


\section{REFERENCES}

1. Anthony, C. 1989. Bacterial oxidation of methane and methanol. Adv. Microb. Physiol. 27:179-183.

2. Cook, S.A., and A.K. Shiemke. 1996. Evidence that copper is a required cofactor for the membrane-bound form of methane monooxygenase. J. Inorg. Biochem. 63:273-284.

3. Nguyen, H.H., S.J. Elliott, J.H. Yip, and S.I. Chan. 1990. The particulate methane monooxygenase from Methylococcus capsulatus (Bath) is a novel copper-containing three-subunit enzyme. Isolation and characterization. J. Biol. Chem. 273:7957-7966.

4. Nielsen, A.K., K. Gerdes, and J.C. Murrell. 1997. Copper-dependent reciprocal transcriptional regulation of methane monooxygenase genes in Methylococcus capsulatus and Methylosinus trichosporium. Mol. Microbiol. 25:399-409.

5. Otten, M.F., W.N.M. Reijnders, J.J.M. Bedaux, H.V. Westerhoff, K. Krab, and R.J.M. Van Spanning. 1999. The reduction state of the Q-pool regulates the electron flux through the branched respiratory network of Paracoccus denitrificans. Eur. J. Biochem. 261:767-774.

6. Umbach, A.L., and J.N. Siedow. 1996. The reaction of the soybean cotyledon mitochondrial cyanide-resistant oxidase with sulfhydryl reagents suggests that $\alpha$ - 
keto acid activation involves the formation of a thiohemiacetal. J. Biol. Chem.

271:25019-25026.

7. Zahn, J.A., and A.A. DiSpirito. 1996. Membrane-associated methane

monooxygenase from Methylococcus capsulatus (Bath). J. Bacteriol. 178:1018-

1029. 


\section{FUTURE WORK}

It is advised that continuing research on this project concentrate on the following five areas:

(i) investigation of oxiadase activities with higher concentrations of cyanide

(ii) purification of the putative alternative oxygen consumer

(iii)purify pMMO with the decyl-plastoquinol as the reducing source

(iv)extension of studies with DMSO as substrate

(v) reconstitution of NDH-2 with the endogenous quinol

The investigations performed here suggested that cyanide does not exert the impact of inhibition of oxygen consumption to the extent that octyl gallate was capable. However, the concentrations of cyanide used here may not be sufficient to provide suffiicient insight. It was later discovered that concentrations as high as $5 \mathrm{mM}$ are typically used to inhibit alternative oxidases in some species.

If it is concluded, unequivocally, that an alternative oxidase exists, purification of this protein would provide more insight into the respiratory function of the chain. Many species contain alternative oxidases which possess simpler subunit stoichiometries than that of the primary oxidase. Following purification, sequencing anlysis could provide information to formulate a 3-dimensional model of the enzyme and suggest potential substrate binding sites, as well as conserved amino acids. 
As supported by evidence in two different chapters within this manuscript, decylplastoquinol may serve as a suitable alternate reductant during purification of pMMO. As mentioned many times, the past failures of pMMO purification may have been the result of dissociation of essential subunits and/or cofactors. If quinols are capable of direct reduction of pMMO (decyl-plastoquinol being the best candidate from this work), then purification with such a substrate should prove useful during in-process analyses.

DMSO was curiously capable of reducing cytochrome $c$ when octyl gallate was used as an inhibitor, but not when cyanide was utilized. However, DMSO showed no apparent increase in oxygen uptake with the oxygen electrode in either the membrane fraction or whole cells. This seems very puzzling an surely requires more investigation and a different series of experiments. One species of methylotroph has been shown to metabolize DMSO as a substrate, but evidence for this has not been shown for $M$. capsulatus to date.

Finally, reconstitution of NDH-2 with endogenous quinone could provide evidence for the interaction of the reductase with endogenous lipids suitable for subsequent reduction of pMMO. Cook, et. al. attempted such an experiment, but only one concentration was investigated. A series of concentrations may provide a better understanding of the true interactions in vivo. 